\title{
GIF NATURAL RADIOCARBON MEASUREMENTS XI
}

GEORGETTE DELIBRIAS and MARIE-THERESE GUILLIER

Centre des Faibles Radioactivités, Laboratoire Mixte CNRS-CEA

Avenue de la Terrasse, 91198-Gif-sur-Yvette Cedex, France

This list is part of a program of publishing our large backlog of unpublished dates. The dates listed here, from 1974 to 1979 , include archaeologic samples from various cultures and countries and geologic samples related to sea-level variations, volcanism, and especially the history of paleolakes in Africa.

Ages are calculated according to the Libby half-life of $5570 \pm 30$ years. The recent standard is $95 \%$ of the ${ }^{14} \mathrm{C}$ activity of oxalic acid, referring to 1950. All ages are given in years before present (AD 1950). Corrections for isotopic fractionations are made only when $\delta^{13} \mathrm{C}$ values are given.

The marine shells in our present and previous lists are corrected for reservoir effect of ca $400 \mathrm{yr}$, since there is no systematic isotopic correction. For lascustrine shells and carbonate formations corrections are more complex, and were performed when $\delta^{13} \mathrm{C}$ values were available.

Some dates were calibrated using the correction table of Klein et al (1982) and are reported as "cal" ages.

\section{ACKNOWLEDGMENTS}

The authors thank M Rousseau and Y San Vicente for sample preparation, and $\mathrm{M}$ Jaudon and J-P Garnier for routine operation of the dating equipment.

\section{ARCHAEOLOGIC SAMPLES}

\section{France}

\section{Les Sablins series, Etaples, Pas de Calais}

Charcoal from fill of pits from flint chipping workshop $\left(50^{\prime} 31^{\prime} \mathrm{N}, 1^{\circ}\right.$ $39^{\prime}$ E). Coll by J Hurtrelle and subm 1975-1977 by J F Piningre. Abundant assoc lithic industry is attributed to Neolithic of "Cerny" type.

Gif-3701. Les Sablins, D II, b 1

$5660 \pm 120$

Gif-4024. Les Sablins, B IV, c 1

$5690 \pm 120$

General Comment: dates Cerny culture and beginning of "Neolithization" in N France (Hurtrelle \& Piningre, 1978).

Gif-3227. Labuissière, Pas de Calais

$1720 \pm 90$

Charcoal from Gallo-Roman potter's workshop $\left(50^{\circ} 30^{\prime} \mathrm{N}, 2^{\circ} 34^{\prime} \mathrm{E}\right)$. Coll and subm 1973 by G Vion, Soc Recherche Hist, Bruay-en-Artois. Comment: date agrees with expectation. 
Gif-3611. Houdain-les-Bavay, Nord

$920 \pm 90$

Charcoal from cave-refuge $\left(50^{\circ} 18^{\prime} \mathrm{N}, 3^{\circ} 48^{\prime} \mathrm{E}\right)$. Coll and subm 1975 by F Ozeel, Maroilles, Nord. Comment: date confirms expected age of ceramics.

Gif-3551. Loos-lez-Lille, Nord

Charcoal from refuse ditch, assoc with atypical ceramics $\left(56^{\circ} 28^{\prime} \mathrm{N}, 3^{\circ}\right.$ $15^{\prime}$ E). Coll and subm 1975 by G Leman, Dir Antiquités Hist, Lille. Comment: date agrees with expected La Tène age.

\section{Neuville-sur-Escaut series, Nord}

Charcoal samples, at base of pits, in terrace of Escaut $\mathrm{R}\left(58^{\circ} 18^{\prime} \mathrm{N}, 3^{\circ}\right.$ $24^{\prime} \mathrm{E}$ ). Coll in La Cimenterie quarry and subm 1975 by G Hantute, Circonscription Antiquités Préhist Nord-Picardie, Valenciennes, Nord. Assoc with some metal pieces and crude ceramics.

Gif-3625. Neuville-sur-Escaut, point A $2310 \pm 120$

Gif-3626. Neuville-sur-Escaut, point B $2430 \pm 110$

Gif-3627. Neuville-sur-Escaut, point $\mathbf{C}$ $2420 \pm 110$

Gif-3728. Neuville-sur-Escaut, point E $2500 \pm 130$

Comment: undersized sample.

General Comment: dates correspond to boundary between Hallstatt and La Tène ages.

\section{Vers-sur-Selle series, Somme}

Samples from collective tomb in passage grave $\left(49^{\circ} 51^{\prime} \mathrm{N}, 2^{\circ} 14^{\prime} \mathrm{E}\right)$. Coll and subm 1975-1982 by J F Piningre, Dir Antiquités Préhist, Villeneuve d'Ascq, Nord.

Gif-3698. Vers-sur-Selle, F 3-4 IV

$3070 \pm 110$

Charcoal from destruction level of monument.

Gif-3699. Vers-sur-Selle, F a-1-1

$4060 \pm 120$

Charcoal in fill of pit.

Gif-3700. Vers-sur-Selle, F a-XII b

$4240 \pm 120$

Charcoal in fill of circular ditch, assoc with Neolithic ceramics of SOM type.

Gif-5740. Vers-sur-Selle, $X$ b

$4240 \pm 100$

Human bones from tomb.

General Comment: Gif-3700 and -5740 agree well with expected age from assoc industry and type of monument (Piningre \& Bréart, 1976). 
Jonquières series, Oise

Bovidae bones from open air site $\left(49^{\circ} 24^{\prime} \mathrm{N}, 2^{\circ} 44^{\prime} \mathrm{E}\right)$. Coll and subm 1972 by J C Blanchet, Centre Recherche Archéol, Compiègne, Oise.

Gif-2918. Jonquières, XVII Ba 3

$2340 \pm 100$

Depth $0.80 \mathrm{~m}$.

Gif-2919. Jonquières XIV So 2

$5120 \pm 130$

From fill of Chassean ditch. Comment: agrees with Ly-2970: $5300 \pm$ 140 obtained for bones (R, 1985, v 27, p 432) and with assoc industry.

\section{Chartrettes series, Seine et Marne}

Charcoal from deep pits in Hallstatt site, in alluvium of Seine R $\left(48^{\circ}\right.$ $29^{\prime} \mathrm{N}, 2^{\circ} 41^{\prime} \mathrm{E}$ ). Coll by J Tarrête and subm 1975 by M Brézillon, Mus Homme, Paris. Assoc with ceramics of Hallstatt period.

Gif-2952. Chartrettes

$2750 \pm 100$

From detritus pit.

Gif-3677. Chartrettes, pit I, B-C

$2450 \pm 100$

Gif-3678. Chartrettes, pit II, G

$2450 \pm 100$

General Comment: dates agree well with assoc ceramics.

\section{Pincevent series, Seine et Marne}

Pincevent, famous open-air Late Magdalenian encampment, is situated on left bank of Seine R $\left(48^{\circ} 23^{\prime} \mathrm{N}, 2^{\circ} 53^{\prime} \mathrm{E}\right)$. Series of occupations were found on sands and gravels of Seine R, interspersed with flood loams. Abundance of reindeer bones suggest that inhabitants were mainly hunters. Samples, except for Gif-3480, coll and subm 1981-1983 by M Julien, Coll France and CNRS, Paris.

Gif-3480. Pincevent, 74, IV 2

$9460 \pm 170$

Ashes from hearth in Level IV 2, coll 1976 by A Leroi-Gourhan, Coll France, Paris.

Gif-6283. Pincevent, IV 2

$$
12,120 \pm 130
$$

Carbonaceous material from hearth in Level IV 2, coll 1982.

Gif-6284. Pincevent, IV 26

$11,800 \pm 130$

Carbonaceous material from hearth in Level IV 26, coll 1981.

Gif-6310. Pincevent IV 30

$12,100 \pm 130$

Carbonaceous material from hearth in Level IV 30, coll 1983.

Gif-5971. Pincevent, IV 40

Charcoal Level IV 40, coll 1982.

$12,100 \pm 120$ 
General Comment: these dates, except for Gif-3480 which is aberrant, with Gif-358: $12,300 \pm 400(\mathrm{R}, 1970, \mathrm{v} 12, \mathrm{p} 430)$ indicate that Pincevent was occupied ca 12,000 BP but probably only during short periods; this supports homogeneity of finds in different occupation levels.

Gif-3750. Argenteuil, Val d'Oise

$870 \pm 90$

Wood from pirogue, in sediment of Oise $\mathrm{R}\left(48^{\circ} 57^{\prime} \mathrm{N}, 2^{\circ} 15^{\prime} \mathrm{E}\right)$. Coll and subm 1975 by L Lherault, Soc Hist \& Archaeol, Argenteuil.

\section{Gif-3329. Guiry-en-Vexin, Val d'Oise}

$3640 \pm 100$

Human bones from collective sepulcher of "SOM" Neolithic type, at Bois Couturier $\left(47^{\circ} 49^{\prime} \mathrm{N}, 1^{\circ} 45^{\prime} \mathrm{E}\right)$. Coll and subm 1974 by M Brezillon. Comment: date is slightly younger than "SOM" Neolithic age expected from ceramics.

Gif-3330. Courcelles-sur-Viosne, Val d'Oise

$4060 \pm 110$

Charcoal from pit, of Middle Neolithic site $\left(49^{\circ} 05^{\prime} \mathrm{N}, 2^{\circ} 00^{\prime} \mathrm{E}\right)$. Coll by $\mathrm{J}$ Degros and J Tarrête and subm 1974 by M Brezillon. Comment: date is younger than expected.

Gif-3000. Palaiseau, Essonne

$1320 \pm 90$

Human bones from burial found in archaeol level under sacristy of St Martin of Palaiseau Church $\left(48^{\circ} 43^{\prime} \mathrm{N}, 2^{\circ} 14^{\prime} \mathrm{E}\right)$. Coll and subm 1974 by J M Bartholi, Palaiseau. Comment: date confirms existence of Gallo-Roman level (Bartholi, 1975).

Gif-3287. Buthiers, Essonne, FA-060

$4760 \pm 110$

Charcoal from Tardenoisian shelter of Chateaubriand $\left(48^{\circ} 18^{\prime} \mathrm{N}, 2^{\circ}\right.$ 26' E). Coll and subm 1974 by J Hinout, Château-Thierry, Aisne.

Gif-3557. Sonchamp, Yvelines

$4940 \pm 120$

Charcoal from Neolithic site of “Augy-Sainte-Pallaye” type $\left(48^{\circ} 35^{\prime} \mathrm{N}\right.$, $\left.1^{\circ} 53^{\prime} \mathrm{E}\right)$. Coll and subm 1974 by J Tarrête, Dir Antiquités Préhist région parisienne, Paris. Comment: date agrees perfectly with age expected from ceramics.

\section{Montigny series, Loiret}

Charcoal from collective tomb $\left(48^{\circ} 07^{\prime} \mathrm{N}, 02^{\circ} 08^{\prime} \mathrm{E}\right)$. Coll and subm 1975 by C Masset, Paris.

Gif-3759. Montigny, D 3-D 2 III $4310 \pm 130$

Gif-3760. Montigny, B 3 III

$4490 \pm 130$

General Comment: dates agree with expected age for this kind of monument. 
Charcoal and ashes from iron foundry settlement $\left(47^{\circ} 58^{\prime} \mathrm{N}, 2^{\circ} 15^{\prime} \mathrm{W}\right)$ Coll and subm 1973 by A Pioger, Le Mans. Comment: this foundry activity is dated cal 545-20 BC.

\section{Plelauff series, Kerivoelen, Côtes du Nord}

Charcoal from megalithic tomb $\left(48^{\circ} 13^{\prime} \mathrm{N}, 3^{\circ} 11^{\prime} \mathrm{W}\right)$. Coll and subm 1975 by C T Le Roux, CNRS, Rennes.

\section{Gif-3586. Plelauff, 1}

Under pavement.

\section{Gif-3587. Plelauff, 2}

$3640 \pm 110$

From funeral room.

General Comment: dates indicate Chalcolithic period, in spite of assoc Late Neolithic ceramics of "SOM" type.

\section{Liscuis en Laniscat series, Côtes du Nord}

Charcoal from group of three megalithic tombs of passage-grave type on sandy moor $\left(48^{\circ} 13^{\prime} \mathrm{N}, 3^{\circ} 08^{\prime} \mathrm{W}\right)$. Coll and subm $1974-1976$ by C T Le Roux.

Gif-3943. Laniscat, 75-1

$2250 \pm 90$

Comment: indicates re-use of monument.

Gif-4075. Laniscat, 76-1

$3680 \pm 110$

From hearth near tomb, at basal mound around tomb. Comment: date suggests re-use of monument during Bronze Age.

Gif-3585. Laniscat, 74-3

From funeral room of outlying building.

Gif-4076. Laniscat, 76-2

Comment: dates building of monument.

Gif-3944. Laniscat, 75-2

Comment: dates building of monument.

General Comment: dates agree well with other dates obtained for these tombs (R, 1986, v 28, no. 1, p 11) and establish chronology of construction of these passage graves.

Gif-3748. Trans, Ille-et-Vilaine

Charcoal from pottery kiln $\left(48^{\circ} 30^{\prime} \mathrm{N}, 1^{\circ} 35^{\prime} \mathrm{W}\right)$. Coll and subm 1975 by L Langouët, Univ Rennes. Comment: younger than expected. 
Gif-3746. Saint-Malo, Ille-et-Vilaine

$1050 \pm 90$

Charcoal from kiln of bronze foundry inside Alet cathedral $\left(48^{\circ} 38^{\prime} \mathrm{N}\right.$, $2^{\circ} 02^{\prime} \mathrm{W}$ ). Coll and subm 1975 by L Langouët. Comment: agrees with expected age from chronology of monument building.

Gif-3747. Erdeven, Morbihan

$4800 \pm 110$

Charcoal from hearth of flint chipping workshop $\left(47^{\circ} 38^{\prime} \mathrm{N}, 3^{\circ} 12^{\prime} \mathrm{W}\right)$. Coll and subm 1975 by C T Le Roux. Comment: site is dated to Neolithic period as expected.

\section{L'Epinette series, Préfailles, Loire Atlantique}

Samples from Camp des Fougerais $\left(47^{\circ} 08^{\prime} \mathrm{N}, 2^{\circ} 15^{\prime} \mathrm{W}\right)$ assoc with “augets" pottery salt pans. Coll by M Tessier and subm by J L'Helgouach, Dir Antiquité Préhist, Nantes.

Gif-821. L'Epinette, I

$1930 \pm 130$

Charcoal, coll 1967.

Gif-3765. L'Epinette, II

$1870 \pm 100$

Marine shells.

General Comment: both dates disagree with Hallstatt age attributed to ceramics.

\section{La Fougerais B series, Saint-Michel-Chef-Chef, Loire Atlantique}

Charcoal from enclosure ditch of promontory Camp $\left(47^{\circ} 09^{\prime} \mathrm{N}, 2^{\circ} 08^{\prime}\right.$ W). Coll by M Tessier and subm 1975 by J L'Helgouach. Assoc with abundant ceramics and remains of pottery kilns.

Gif-3533. Les Fougerais, B I

$1890 \pm 90$

Comment: dates Late Iron Age ceramics (Tessier, 1983).

Gif-3534. Les Fougerais, B III

$2300 \pm 100$

Gif-3535. Les Fougerais, B 5

$2300 \pm 100$

General Comment: Gif-3534-3435 confirm first settlement of site at 4 th century $\mathrm{BC}$, as expected from ceramics (Tessier, 1983).

\section{La Pierre Couvretière series, Ancenis, Loire Atlantique}

Human bones found in Neolithic dolmen $\left(47^{\circ} 22^{\prime} \mathrm{N}, 1^{\circ} 18^{\prime} \mathrm{W}\right)$. Coll by D Prigent and subm 1974-1975 by J L'Helgouach.

Gif-3415. La Pierre Couvretière, I $2990 \pm 110$

Gif-3763. La Pierre Couvretière, II

$2880 \pm 100$

General Comment: dates indicate re-use of monument, confirmed by presence of protohistoric potsherds. 
Gif-3536. Hêle, Donges, Loire Atlantique

$4230 \pm 110$

Wood, at foot of menhir $\left(47^{\circ} 22^{\prime} \mathrm{N}, 2^{\circ} 05^{\prime} \mathrm{W}\right)$, overlying blue clay and underlying peat, $0.50 \mathrm{~m}$ deep. Coll by D Prigent and subm 1974 by J L'Helgouach. Comment: date agrees with expected age of monument, but disagrees with presence of Subatlantic pollens in clay.

Gif-3532. Fossé de Gatineau, Saint-Michel-

Chef-Chef, Loire Atlantique

$4900 \pm 110$

Charcoal from ditch of Neolithic camp $\left(47^{\circ} 10^{\prime} \mathrm{N}, 2^{\circ} 08^{\prime} \mathrm{W}\right)$. Coll by M Tessier and subm 1975 by J L'Helgouach. Comment: archaeol material corresponds to Late Neolithic. Date suggests earlier occupation.

\section{Gif-3531. Pointe Saint-Gildas, Préfailles,} Loire Atlantique

$7520 \pm 140$

Shells from refuse pit of Mesolithic site $\left(47^{\circ} 08^{\prime} \mathrm{N}, 2^{\circ} 15^{\prime} \mathrm{W}\right)$. Coll by $\mathrm{M}$ Tessier and subm 1975 by J L'Helgouach. Assoc with microliths. Comment: very good date for typical archaeol material of Middle Tardenoisian.

\section{Melleran series, Sauzé-Vaussais, Deux-Sèvres}

Charcoal from artificial souterrain $\left(46^{\circ} 07^{\prime} \mathrm{N}, 0^{\circ} 00^{\prime} \mathrm{W}\right)$. Coll and subm 1975 by R Proust, Soc Hist Deux-Sèvres. These souterrains, numerous in region, are probably ancient refuges.

Gif-3457. Melleran, no. 1

$780 \pm 90$

From entrance passage.

Gif-3669. Melleran, no. 2

From Pit A.

General Comment: dates correspond to two different occupations of souterrain.

\section{Camp Allaric series, Aslonnes, Vienne}

Charcoal samples from boring VI in open-air site $\left(46^{\circ} 28^{\prime} \mathrm{N}, 0^{\circ} 22^{\prime} \mathrm{E}\right)$. Coll and subm 1973 by J Pautreau.

Gif-3008. Camp Allaric, Boring VI-190

$2560 \pm 140$ ics.

At basal level of Hallstatian rampart, assoc with Late Bronze III ceram-

Gif-3009. Camp Allaric, Boring VI-280

$4260 \pm 140$

Assoc with potsherds of Artenac type.

Gif-3010. Camp Allaric, Boring VI-310

$4280 \pm 140$

Assoc with Chalcolithic artifacts.

General Comment: Gif-3009 and -3010 date Artenac culture which corresponds to main Chalcolithic occupation in W central France (Pautreau, 1975). 


\section{Martizay series, Indre}

Wood from ancient pilings of bridge at bottom of Claise $\mathrm{R}\left(46^{\circ} 49^{\prime} \mathrm{N}\right.$, $\left.1^{\circ} 03^{\prime} \mathrm{E}\right)$. Coll and subm 1974 by M Hours, Lab Mus France, Palais Louvre, Paris.

Gif-3067. Martizay, 3

$$
200 \pm 90
$$

Gif-3068. Martizay, 2

$$
560 \pm 90
$$

General Comment: results do not date bridge, which is Gallo-Roman, but restoration periods.

\section{Gif-3256. Saint-Martial de Mirambeau,} Charente Maritime

$1570 \pm 90$

Charcoal from protohistoric ditch discovered by aerial photography at "La Champagne de Font Tertaud" ( $\left.45^{\circ} 21^{\prime} \mathrm{N}, 0^{\circ} 37^{\prime} \mathrm{W}\right)$. Subm 1974 by J Dassié, Versailles.

Gif-3422. La Prevalerie de Dirac, Charente

$$
2700 \pm 110
$$

Peat in Aurochs cranium, $1.4 \mathrm{~m}$ deep in peat bog $\left(45^{\circ} 35^{\prime} \mathrm{N}, 0^{\circ} 13^{\prime} \mathrm{W}\right)$. Coll and subm 1974 by J Massaud, Angoulême. Whole skeleton was found nearby. Comment (TM): agrees with assoc post-Neolithic industry. Aurochs disappeared from France ca AD 13th century.

Gif-3609. Roc de Sers, Charente

Bones from Early Solutrean level of prehistoric station with rupestral art $\left(45^{\circ} 36^{\prime} \mathrm{N}, 0^{\circ} 42^{\prime} \mathrm{W}\right)$. Coll and subm 1975 by G Henri Martin, CNRS, Paris. Comment: date agrees well with assoc Early Solutrean lithics.

\section{Saline de Dousseille series, Guérande, Vendée}

Samples from various archaeol sites, along littoral, near Guérande $\left(47^{\circ}\right.$ $19^{\prime} \mathrm{N}, 2^{\circ} 28^{\prime} \mathrm{W}$ ).

\section{Gif-3764. Saline de Dousseille, I}

$$
1750 \pm 100
$$

Charcoal from habitation site with "augets" pottery salt pans. Coll by D Prigent and subm 1975 by J L'Helgouach. Comment: in expected range of date.

Gif-4120. Saline de Dousseille, II

$610 \pm 90$

Human bones from necropolis. Coll by J Y Gallais and subm 1976 by J L'Helgouach. Comment: dates recent occupation of site.

\section{Gif-3417. Les Aspies, Nieul-sur-l'Autize,}

$$
\text { Vendée }
$$

$4040 \pm 130$

Fragment of human cranium assoc with Campaniforme Chalcolithic industry, from dolmen under tumuli $\left(46^{\circ} 27^{\prime} \mathrm{N}, 0^{\circ} 41^{\prime} \mathrm{E}\right)$. Coll by $\mathrm{R}$ Joussaume and subm 1973 by J L'Helgouach. Comment ( J L'H): very good date for Atlantic Campaniform occupation. 
Gif-3761. PetitRocher, Brétignolles, Vendée

$4290 \pm 130$

Charcoal from coastal Chalcolithic habitat in sandy dune $\left(46^{\circ} 37^{\prime} \mathrm{N}, 1^{\circ}\right.$ $53^{\prime} \mathrm{W}$ ). Coll by R Joussaume and subm 1975 by J L'Helgouach. Assoc with rich Chalcolithic industry, ceramics and gold jewelry. Comment: date is exactly as expected.

\section{Pierre Virante II series, Xanton Chassenon, Vendée}

Human bones from typical megalithic monument $\left(46^{\circ} 28^{\prime} \mathrm{N}, 0^{\circ} 41^{\prime} \mathrm{E}\right)$. Coll by R Joussaume and subm 1974-1975 by J L'Helgouach.

Gif-3416. Pierre Virante, I $4870 \pm 140$

Gif-3762. Pierre Virante, II

$4040 \pm 130$

General Comment: date for 2nd sample, Gif-3762, is exactly as expected, whereas Gif-3416 is much too old and difficult to explain.

\section{Gif-3675. Nieul sur l'Autize, Vendée}

$4110 \pm 130$

Charcoal from Neolithic camp with triple surrounding walls $\left(46^{\circ} 25^{\prime} \mathrm{N}\right.$, $\left.0^{\circ} 39^{\prime} \mathrm{W}\right)$. Coll and subm 1975 by R Joussaume, Les Sables d'Olonne, Vendée. Comment: in expected age range.

Gif-3676. Les Cous, Bazoges-en-Pareds, Venolée

$4680 \pm 130$

Charcoal from passage grave $\left(46^{\circ} 39^{\prime} \mathrm{N}, 3^{\circ} 16^{\prime} \mathrm{E}\right)$. Coll and subm 1975 by R Joussaume. Comment: in expected age range.

\section{Gif 3649. La Faurélie II, Dordogne}

$11,780 \pm 180$

Carbonized bones from hearth of Magdalenian V Level of La Faurélie rock shelter $\left(44^{\circ} 56^{\prime} \mathrm{N}, 1^{\circ} 02^{\prime} \mathrm{E}\right)$. Coll 1966 and subm 1975 by J Tixier, CNRS, Meudon-Bellevue. Comment: date is rather young for assoc Magdalenian $\mathrm{V}$ industry assoc. Bones were probably slightly contaminated.

\section{Crozo Bastido series, Pinsac, Lot}

Charcoal from cavern $\left(44^{\circ} 51^{\prime} \mathrm{N}, 1^{\circ} 30^{\prime} \mathrm{E}\right)$. coll and subm 1974 by $\mathrm{M}$ Lorblanchet, CNRS, Paris.

\section{Gif-3279. Crozo Bastido, Upper Level II$$
2230 \pm 110
$$ \\ Gif-3280. Crozo Bastido, Level III \\ $2710 \pm 110$}

General Comment: date of Level II agrees with Iron Age date of ceramics, but date of Level III disagrees with expected Middle Bronze Age date probably due to numerous observed disturbances of site.

\section{Cuzoul series, Vers, Lot}

Cuzoul Rock Shelter $\left(44^{\circ} 27^{\prime} \mathrm{N}, 1^{\circ} 32^{\prime} \mathrm{E}\right)$, offers very interesting stratigraphy, $3 \mathrm{~m}$ thick with 50 levels of successive temporary occupations during Early Magdalenian (Clottes \& Giraud, 1985). Samples coll and subm 198283 by $J$ Clottes, Dir Antiquités Préhist Midi-Pyrénées, Foix. 
Gif-6372. Cuzoul, Level 3, a-b

$$
14,560 \pm 130
$$

Bone splinters.

Gif-6638. Cuzoul, Level 5 c

$$
15,980 \pm 150
$$
level.

Bone splinters, mainly reindeer. Corresponds to Magdalenian II

Gif-6371. Cuzoul, Level 13

$16,800 \pm 170$

Bone splinters from Middle Classic Magdalenian I level.

Gif-6797. Cuzoul, Level 20

$17,050 \pm 170$

Bone splinters from base of Magdalenian I level.

Gif-6370. Cuzoul, Level 23

$18,300 \pm 200$

Bone splinters from Magdalenian O level.

Gif-6798. Cuzoul, Level 24

$18,400 \pm 200$

Bones from very early Magdalenian O level.

Gif-6699. Cuzoul, Level 30

$19,400 \pm 210$

Bone splinters from basal level with typical Solutrean tools.

General Comment: due to thickness of sequence, high-precision dating of Early Magdalenian might be performed.

\section{Grotte des Escabasses series, Thémines, Lot}

Charcoal from occupation levels $\left(44^{\circ} 45^{\prime} \mathrm{N}, 1^{\circ} 48^{\prime} \mathrm{E}\right)$. Coll and subm 1974 by M Lorblanchet.

Gif-3276. Grotte des Escabasses, Upper Level III

$2710 \pm 110$

Late Bronze-Early Iron Age occupation level.

Gif-3277. Grotte des Escabasses, Base Level III

Late Bronze-Early Iron Age occupation.

Gif-3278. Grotte des Escabasses, Level VIc

$4120 \pm 120$

Late Neolithic occupation.

General Comment: dates fit well with expected ages. Gif-3276 and -3277 seem inverted, yet are almost in statistical range of each other.

\section{Abri du Mas Viel series, Saint-Simon, Lot}

Bone splinters from famous Mousterian shelter, Mas Viel $\left(44^{\circ} 42^{\prime} \mathrm{N}\right.$, $\left.1^{\circ} 50^{\prime} \mathrm{N}\right)$. Coll and subm 1974 by M Lorblanchet.

Gif-3281. Mas Viel, Upper Level C

$26,770 \pm 800$

Gif-3559. Mas Viel, Level E

$>40,000$ 
General Comment: lithics were studied by F Bordes after excavation in 1954 . $\mathrm{He}$ attributed them to peculiar Late Mousterian though date of Level $\mathrm{C}$ is too young.

\section{Sargel Cave series, Saint-Rome de Cernon, Aveyron}

Charcoal from different levels of Sargel Cave $\left(44^{\circ} 01^{\prime} \mathrm{N}, 2^{\circ} 59^{\prime} \mathrm{W}\right)$.

Coll 1967 and subm 1973 by G Costantini, Millau, Aveyron.

Gif-3005. Sargel Cave, Level V

$3800 \pm 130$

Level attributed to Chalcolithic period.

Gif-3006. Sargel Cave, Level IX

$3620 \pm 130$

Level attributed to Chalcolithic period.

Gif-3007. Sargel Cave, Level XIII

Level attributed to Early Chassean period.

$4650 \pm 150$

General Comment: dates are slightly younger than expected.

Gif-3502. Tumulus de Lardicou, Cancalières, Tarn

$2140 \pm 90$

Charcoal from hearth of stone tumulus $\left(43^{\circ} 31^{\prime} \mathrm{N}, 2^{\circ} 19^{\prime} \mathrm{E}\right)$. Coll and subm 1975 by M Labrousse, Antiquités Hist Midi-Pyrénées. Assoc with potsherds of Gallo-Roman appearance. Comment: site older than presumed age.

La Poujade series, Millau, Aveyron

Charcoal from rock shelter $\left(44^{\circ} 07^{\prime} \mathrm{N}, 3^{\circ} 10^{\prime} \mathrm{E}\right)$. Coll and subm $1974-$ 75 by $\mathrm{G}$ B Arnal, CNRS, Montpellier.

Gif-3418. La Poujade, Level $10 \mathrm{C}$ $8710 \pm 190$

Gif-3631. La Poujade, Level I D

$8990 \pm 190$

General Comment: assoc lithic material seems Mesolithic and agrees well with date, but presence of potsherds in levels so old suggests disturbance.

Gif-3419. Grotte du Bourrut, Sumène, Gard

$8790 \pm 190$

Charcoal from Chassean level $\left(43^{\circ} 50^{\prime} \mathrm{N}, 3^{\circ} 40^{\prime} \mathrm{E}\right)$. Coll and subm by $\mathrm{G}$ B Arnal. Comment: date is 3000 yr older than industry and remains unexplained.

Gif-3322. Prades, Lozère

$4010 \pm 120$

Carbonized wheat in "Fontbouisse" Chalcolithic level, from Aven des Corneilles $\left(44^{\circ} 18^{\prime} \mathrm{N}, 3^{\circ} 27^{\prime} \mathrm{E}\right)$. Coll and subm 1974 by G Fages, CNRS, Thémines, Lot. Comment: dates beginning of "Fontbouisse" culture in "Grandes Causses," Massif Central.

\section{Gif-3648. Memer, Vailhoules, Aveyron}

Bones of "Man of Memer" $\left(44^{\circ} 20^{\prime} \mathrm{N}, 1^{\circ} 53^{\prime} \mathrm{E}\right)$ found in 1948 and subm 1975 by M Delhon-Bujard, Toulouse. Comment: dates skeleton of man, antiquity of whom has been much debated. 
Gif-3704. Château de Montaner, Hautes Pyrénées

Wood from stake in well filling $\left(43^{\circ} 20^{\prime} \mathrm{N}, 0^{\circ} 00^{\prime}\right)$. Subm 1976 by $\mathrm{D}$ Bardin.

Gif-3701. Germs-sur-L'Oussouet, Hautes Pyrénées

$2480 \pm 100$

Wood from timbers of argentiferous galena workings $\left(43^{\circ} 03^{\prime} \mathrm{N}, 0^{\circ} 03^{\prime}\right.$ E). Coll and subm 1976 by D Bardin, ELF Aquitaine Soc, Pau.

\section{Saint-Jean-de-Verges series, Ariège}

Faunal bones from unsheltered site near little cave $\left(43^{\circ} 01^{\prime} \mathrm{N}, 1^{\circ} 36^{\prime}\right.$ E). Coll 1964 by J Vezian and subm 1973 by J Bouchud, Mus d'Hist Nat, Paris.

Gif-2942. Saint-Jean-de-Verges, 1973-1

$21,500 \pm 400$

From Perigordian V Level.

Gif-2941. Saint-Jean-de-Verges, 1973-2 $24,200 \pm 600$

From Aurignacian I Level separated from Perigordian V level by red clayey layer, 0.10 to $0.20 \mathrm{~m}$ thick.

General Comment: Gif-2942 agrees well with expected age of Perigordian V, whereas Gif-2941 is much too young for assoc Aurignacian I industry, probably because of recent contamination of bones.

Gif-2943. Grottes du Portel, Ariège

$12,760 \pm 170$

Bone fragments in Magdalenian IV hearth, underlying sterile red clay and stalagmitic deposits $\left(43^{\circ} 01^{\prime} \mathrm{N}, 1^{\circ} 36^{\prime} \mathrm{E}\right)$. Coll 1972 by $\mathrm{J}$ Vezian and subm 1973 by J Bouchud. Comment: data is a little too young but does not disagree with range of expected ages from industry.

Gif-2950. La Cauna, Belvis, Aude

$12,270 \pm 280$

Bones from Level $3\left(42^{\circ} 55^{\prime} \mathrm{N}, 2^{\circ} 23^{\prime} \mathrm{E}\right)$. Coll and subm 1973 by D Sacchi, Lab Préhist CNRS, Caracassonne. Comment: date agrees perfectly with assoc Magdalenian VI industry.

Gif-2981. Lassac, Sallèles-Cabardès, Aude

$16,750 \pm 250$

Faunal bone fragments from Level IIb of open air site $\left(43^{\circ} 18^{\prime} \mathrm{N}, 2^{\circ}\right.$ 23' E). Coll and subm 1973 by D Sacchi. Comment: (DS) date agrees perfectly for Early Magdalenian site, first open-air Magdalenian site known in $\mathrm{S}$ France and probably most noteworthy one found in situ for this period (Sacchi, 1973).

Grotte d'Enlène series, Montesquieu-Avantès, Ariège

Grotte d'Enlène $\left(43^{\circ} 02^{\prime} \mathrm{N}, 1^{\circ} 13^{\prime} \mathrm{E}\right)$ is cave in famous group of caverns of Volp R (Clottes, 1985). Samples were subm 1976-1984 by J Clottes.

Gif-5319. Grotte d'Enlène 0-9

$\mathbf{8 9 0} \pm \mathbf{8 0}$

Charcoal from superficial hearth in base of cave. Comment: dates recent occupation of cave. 
Gif-4122. Grotte d'Enlène, Level 1-b

Charcoal from little hearth lying on stalagmitic floor. Coll 1976. Comment: dates recent occupation of cave.

Gif-4123. Grotte d'Enlène, Level 3-1

$$
10,080 \pm 230
$$

Charcoal from top of Level 3, from Salle des Morts. Coll 1976. Comment: date disagrees with expected Middle Magdalenian age. Undersized sample.

Gif-4124. Grotte d'Enlène, Level 3-2

$13,940 \pm 250$

Charcoal from thick hearth at base of Level 3, in Salle des Morts. Coll 1976. Assoc with Magdalenian assegais of Lussac-Angles type. Comment: date agrees well with expected age.

Gif-6030. Grotte d'Enlène, 82-416

$13,900 \pm 120$

Carbonized bones from an other hearth of Level 3, in Salle des Morts, in same part of cave as Gif-4124.

Gif-5320. Grotte d'Enlène, Level 1-2

$9630 \pm 170$

Burned bones from Level 1, lying on stalagmitic floor, from Salle du Fond. Coll 1980.

Gif-5321. Grotte d'Enlène, Level 3-e

$12,800 \pm 140$

Burned bones, separated from upper Level 1-2 by thick stalagmitic layer, in Salle du Fond. Coll 1980. Comment: very famous engraved "Plaquette of Enlène" was discovered in this cave 50 yr ago (Begouen et al, 1984). Additional pieces of same plaquette were assoc with bones at base of Level 3-e.

Gif-5770. Grotte d'Enlène, Level 3-f

$13,400 \pm 120$

Bones from Level 3-f of Middle Magdalenian IV in Salle du Fond. Coll 1982. Comment: industry is a little more recent than that from Salle des Morts. Difference between dates is compatible with observed difference between artifacts.

Gif-6655. Grotte d'Enlène, Level 4 $21,000 \pm 250$

Bones from Level 4, near entrance of cave. Coll 1984. Comment: date corresponds to Late Perigordian.

Gif-6656. Grotte d'Enlène, Level 5

$$
\mathbf{2 4 , 6 0 0} \pm \mathbf{3 5 0}
$$

Bones from Level 5, near entrance of cave. Coll 1984. Assoc with typical Perigordian V industry. Comment: date is within ages generally accepted for Late Perigordian.

General Comment: upper levels seem disturbed and ages are too early; dates of lower levels agree well with archaeol attribution.

\section{Gif-3484. Serralongue, Pyrénées Orientales}

Modern

Human bones from necropolis of Late Bronze-Hallstatt transition $\left(43^{\circ}\right.$ $\left.23^{\prime} \mathrm{N}, 2^{\circ} 20^{\prime} \mathrm{E}\right)$. Coll and subm 1974 by H Baills. Comment: dates recent inhumation. 


\section{Can-Pey series, Montferrer, Pyrénées Orientales}

Samples from Late Neolithic level of Can-Pey site $\left(42^{\circ} 53^{\prime} \mathrm{N}, 1^{\circ} 46^{\prime} \mathrm{E}\right)$. Coll and subm 1974-1977 by H Baills, Centre Catalan Etudes Préhist, Cabestany.

Gif-3282. Can-Pey, Level N 3-1

Human bones from sepulcher.

Gif-4045. Can-Pey, Level N 3-2

Charcoal from habitation level. Comment: undersized sample.

Gif-4280. Can-Pey, Level N 3, 18 H 8

$2680 \pm 100$

Charcoal from habitation level $\mathrm{N} 3$.

General Comment: Gif-3282 and -4045 agree with archaeol estimate, but Gif4280 is much too young, which may be explained by intrusion of charcoal from upper level of Late Bronze III A age.

Gif-3787. En Gorner, Villefranche de Conflent, Pyrénées Orientales

$4610 \pm 110$

Charcoal from cave, $\left(42^{\circ} 35^{\prime} \mathrm{N}, 2^{\circ} 22^{\prime} \mathrm{E}\right)$. Coll and subm 1975 by $\mathrm{H}$ Baills. Comment: dates Middle Neolithic of "Groupe de Montbolo" type.

Gif-3346. Vingrau, Pyrénées Orientales

$5300 \pm 110$

Charcoal in hearth from site in cave $\left(42^{\circ} 51^{\prime} \mathrm{N}, 2^{\circ} 48^{\prime} \mathrm{E}\right)$, depth: $0.25 \mathrm{~m}$. Coll and subm 1974 by P Campmajo, Cabestany, Pyrénées Orientales. Assoc with Late Magdalenian VI lithic industry. Comment: aberrant date proves contamination of this level which is too superficial.

Gif-3753. “Las Conques,” Vingrau, Pyrénées Orientales, D 27-C 3

$8780 \pm 120$

Bones from Level 3 from Magdalenian level of prehistoric cave $\left(42^{\circ} 57^{\prime}\right.$ $\mathrm{N}, 2^{\circ} 47^{\prime} \mathrm{E}$ ). Coll and subm 1975 by D Sacchi. Comment: date strongly disagrees with expected age, probably because of contamination of bones.

Gif-3571. Pereiras, Pouzols, Aude

$$
4690 \pm 110
$$

Bones from burial in Chassean ditch $\left(43^{\circ} 17^{\prime} \mathrm{N}, 2^{\circ} 51^{\prime} \mathrm{E}\right)$. Coll 1959 and subm 1975 by G Camps, LAPEMO, Aix-en-Provence. Comment: good date for Late Chassean site.

Gif-3573. Saint-Jean-de-Cas, Mailhac, Aude

$$
3730 \pm 110
$$

Charcoal from Bronze Age site, type Verazian (43 $\left.19^{\prime} \mathrm{N}, 2^{\circ} 49^{\prime} \mathrm{E}\right)$. Coll by P Ambert and coll 1975 by G Camps. Comment: very good date for Verazian culture.

\section{Shelter Jean Cros series, Labastide-en-Val, Aude}

Samples from shelter from Early Neolithic-Mesolithic transition $\left(43^{\circ}\right.$ $05^{\prime} \mathrm{N}, 2^{\circ} 28^{\prime} \mathrm{E}$ ). Coll and subm 1975 by J Guilaine, CNRS, Carcassonne. Assoc with printed ceramics. 
Gif-3575. Shelter Jean Cros, Level 2 b-c

$6600 \pm 130$

Charcoal from Sq IX-6 and X-6.

Gif-3576. Shelter Jean Cros,

Level 2 a-b-c

$7160 \pm 130$

Shells (Helix nemoralis) from Sq VI-4. Comment: may be slightly contaminated by old carbonate.

General Comment: dates confirm previous date for Level 2: $6500 \pm 300$, Gif$218(\mathrm{R}, 1966, \mathrm{v} 8, \mathrm{p} 85)$ and agree well with archaeol estimate (Guilaine, 1979).

Gif-3448. Grotte de Canecaude I, Villardonnel, Aude

$12,300 \pm 600$

Coprolites from Level C ( $\left.43^{\circ} 19^{\prime} \mathrm{N}, 2^{\circ} 19^{\prime} \mathrm{E}\right)$. Coll and subm 1975 by D Sacchi. Comment (DS): stratigraphic position of Level C, between Aurignacian Level III: 22,980 \pm 330 (Gif-2709) and Aurignacian Level IV: 25,510 (Gif-2710) ( $R, 1982, v 24, p$ 323), is unquestionable (Sacchi, 1973). Date strongly disagrees with expected age and remains unexplained.

Gif-3572. Fournes 2, Siran, Hérault

$3140 \pm 110$

Bones from upper Level B, assoc with Campaniform potsherds, in dolmen $\left(43^{\circ} 20^{\prime} \mathrm{N}, 2^{\circ} 40^{\prime} \mathrm{E}\right)$. Coll by P Ambert and coll 1975 by G Camps. Comment: date is too young, but grave goods suggest that levels were disturbed.

Gif-3570. Parignoles, La Livinière, Hérault

$3600 \pm 110$

Bones from classic Early Bronze site, type Verazian $\left(43^{\circ} 18^{\prime} \mathrm{N}, 2^{\circ} 38^{\prime}\right.$ E). Coll by P Ambert and subm 1975 by G Camps. Comment: date agrees with expected age.

\section{Grotte de Camprafaud series, Ferrière-Poussarou, Hérault}

Charcoal samples from different levels of Grotte de Camprafaud $\left(43^{\circ}\right.$ $26^{\prime} \mathrm{N}, 2^{\circ} 54^{\prime} \mathrm{E}$ ). Coll and subm $1974-1975$ by G Rodriguez, Saint Chinian, Pardaillon, and 1976 by J L Roudil, Montpellier.

Gif-3073. Grotte de Camprafaud, Level $2 \quad 3980 \pm 100$

From Level 2, assoc with Chalcolithic industry from "Early BronzeCampaniforme" type.

Gif-3074. Grotte de Camprafaud, Level $3 \quad 4060 \pm 100$

From Level 3, assoc with Chalcolithic industry of "Verazian" type.

\section{Gif-3075. Grotte de Camprafaud, Level 4}

$$
2070 \pm 90
$$

From Level 4, assoc with Chalcolithic industry of "Verazian" type. Comment: date indicates important recent contamination, explained by presence of burrows. $C f$ Gif-3782. 
Gif-3782. Grotte de Camprafaud, Level 4b

$3980 \pm 110$

From Level 4, similar to Gif-3075. Comment: date agrees well with assoc Chalcolithic industry.

Gif-3076. Grotte de Camprafaud, Level 7

$4380 \pm 110$

From Level 7, assoc with Late Neolithic industry of "Tardo-Saintponian" type.

Gif-3469. Grotte de Camprafaud, Level 10

$4950 \pm 110$

From hearth in upper part of Level 10, assoc with Late Neolithic industry of "Saintponian" type.

Gif-3470. Grotte de Camprafaud, Level 10

$$
4920 \pm 110
$$

From same Level as Gif-3469.

Gif-3078. Grotte de Camprafaud, Level 19

$6480 \pm 130$

From Level 19, assoc with Early Neolithic of "Languedocian" type.

Gif-3077. Grotte de Camprafaud, Level 20

$7900 \pm 150$

From Level 20, assoc with Early Neolithic industry. Comment: date is probably oldest for Early Neolithic.

General Comment: dates agree well with assoc archaeol industry. With previously pub dates (R, 1972, v 14, p 285), they confirm long use of cave: 4500 yr of continuous settlement with 20 very well-stratified levels (Rodriguez, 1976, 1983).

\section{Gif-2868. Abri de la Madeleine, Bedoin,}

\section{Vaucluse}

$3020 \pm 100$

Human bones from burial chamber of Chalcolithic dolmen $\left(44^{\circ} 07^{\prime} \mathrm{N}\right.$, $5^{\circ} 10^{\prime}$ E). Coll and subm 1973 by G Sauzade, Dir Antiquités Préhist Provence, Avignon. Comment: younger than expected, but assoc industry is not typical enough for good archaeol date.

\section{Gif-2869. Dolmen de Prignon, Saint-Cézaire Alpes Maritimes}

$4040 \pm 110$

Human bones from basal level $\left(43^{\circ} 38^{\prime} \mathrm{N}, 6^{\circ} 48^{\prime} \mathrm{E}\right)$. Coll and subm 1973 by G Sauzade. Comment: agrees with assoc industry and expected age for that monument.

\section{Capula station series, Levie, Corse}

Charcoal from Rockshelter 1 from important site of Capula $\left(41^{\circ} 42^{\prime} \mathrm{N}\right.$, $9^{\circ} 07^{\prime} \mathrm{E}$ ). Coll and subm 1975 by $\mathrm{F}$ de Lanfranchi, Inst Etudes Corses Préhist, Ajaccio.

Gif-3529. Capula 1

$$
2960 \pm 100
$$

Level VI b, Iron Age. 
Gif-3530. Capula 2

$3410 \pm 100$

Level VII, Hearth F 1, Late Bronze Age.

Gif-4033. Capula 3

Level VIII, Bronze Age.

$3400 \pm 110$

General Comment: dates are in expected range.

Gif-3337. Massiac, Cantal

$220 \pm 90$

Wood in gallery of ancient silver mine $\left(50^{\circ} 16^{\prime} \mathrm{N}, 3^{\circ} 14^{\prime} \mathrm{E}\right)$. Coll and subm 1974 by M Lasserre, Fac Sci, Clermont-Ferrand, Puy de Dôme. Comment: dates recent working.

Gif-3045. Aulnat, Gandaillat, Puy de Dôme

$2150 \pm 100$

Charcoal at bottom of Pit B 29, at Aulnat ( $45^{\circ} 47^{\prime}$ N, $3^{\circ} 05^{\prime}$ E). Coll and subm 1973 by H Pelletier, Univ Clermont-Ferrand. Assoc with Early La Tène I industry. Comment: date agrees with expected age.

\section{Champsemard series, Tournus, Saône et Loire}

Charcoal from La Tène II levels of Celtic site $\left(45^{\circ} 33^{\prime} \mathrm{N}, 4^{\circ} 55^{\prime} \mathrm{E}\right)$. Coll and subm 1974 by M Perrin, Tournus.

Gif-3554. Champsemard, Level 7 $2150 \pm 90$

Gif-3555. Champsemard, Level 4 $2190 \pm 90$

General Comment: dates agree with archaeol data.

Camp de Myard series, Vitteaux, Côte d'Or

Charcoal from fortified Neolithic oppidum $\left(47^{\circ} 23^{\prime} \mathrm{N}, 4^{\circ} 31^{\prime} \mathrm{E}\right)$. Coll and subm 1974 by J P Nicolardot, CNRS, Saint Germain-en-Laye.

Gif-3380. Camp de Myard, 1378

$4500 \pm 130$

From habitation structure.

Gif-3381. Camp de Myard, 1377

$4990 \pm 130$

From Neolithic rampart.

Gif-3670. Camp de Myard, 1522

From habitation structure.

General Comment: dates agree well with previous dates for other structures at same site (R, 1974, v 16, p 35) (Delattre \& Nicolardot, 1976).

\section{Mont Avrollot series, Saint-Florentin, Yonne}

Charcoal samples from Level $9 \mathrm{c}$ in La Tène oppidum $\left(48^{\circ} 0^{\prime} \mathrm{N}, 3^{\circ} 41^{\prime}\right.$ E). Coll and subm 1974 by A Duval, Mus Antiquités Nat, Saint Germainen-Laye.

Gif-3356. Mont Avrollot, E 3

$2290 \pm 110$ 


\section{Gif-3357. Mont Avrollot, E 4}

Gif-3354. Chichery, Yonne

Human bones from Neolithic sepulcher $\left(47^{\circ} 55^{\prime} \mathrm{N}, 3^{\circ} 31^{\prime} \mathrm{E}\right)$. Coll and subm 1974 by J Rigaud, Appoigny, Yonne. Comment: agrees with assoc grave goods.

Gif-3783. Hallignicourt, Haute-Marne

$$
810 \pm 90
$$

Human bones from burial ditch $3\left(48^{\circ} 39^{\prime} \mathrm{N}, 4^{\circ} 52^{\prime} \mathrm{E}\right)$. Coll and subm 1975 by L Lepaje, Saint-Dizier. No artifacts were assoc, but in nearby grave, ceramics of Bronze-Hallstatt transition were found. Comment: date indicates re-use of burial, as suspected.

Gif-3784. Laneuville au Pont, Haute-Marne

$$
2240 \pm 100
$$

Charcoal in refuse pit of habitat $\left(48^{\circ} 38^{\prime} \mathrm{N}, 4^{\circ} 51^{\prime} \mathrm{E}\right)$. Coll and subm 1975 by L Lepaje. Assoc with Early Hallstatt ceramics. Comment: date is younger than expected.

\section{Gif-3785. Nijon-La Mottote, Haute-Marne}

$2020 \pm 100$

Charcoal from hearth at base of barrow $\left(48^{\circ} 12^{\prime} \mathrm{N}, 5^{\circ} 36^{\prime} \mathrm{E}\right)$. Coll and subm 1975 by L Lepaje. Comment: younger than Middle Hallstatt expected.

Gif-3716. Moncey, Doubs

$$
610 \pm 90
$$

Wood from whole dug-out canoe, $5 \mathrm{~m}$ long, in bed of Ogon R $\left(47^{\circ} 17^{\prime}\right.$ $\left.\mathrm{N}, 6^{\circ} 07^{\prime}\right)$. Coll and subm 1975 by P Petrequin, Antiquités Préhist FrancheComté, Besançon.

\section{Gigot series, Bretonvilliers, Doubs}

Charcoal in hearths from habitat at foot cliff $\left(47^{\circ} 13^{\prime} \mathrm{N}, 6^{\circ} 41^{\prime} \mathrm{E}\right)$. Coll and subm 1974 by D Vuillat, Circonscription Antiquités Préhist FrancheComté, Besançon.

\section{Gif-3342. Gigot, K 6-F 1}

$4100 \pm 110$

From pit of Early Bronze Age occupation.

Gif-3343. Gigot, L 6-F 2

$5080 \pm 100$

From Late Tardenoisian Mesolithic-Early Neolithic occupation. General Comment: both samples come from same level: dates indicate that hearth Gif-3342 is intrusive in Tardenoisian level.

Gif-3328. Juniville, Ardennes

$2400 \pm 180$

Carbonized plant remains from La Tene I site $\left(49^{\circ} 24^{\prime} \mathrm{N}, 4^{\circ} 21^{\prime} \mathrm{E}\right)$. Coll and subm 1974 by J L Flouest, Saint Dizier, Haute Marne. Comment: agrees well with ceramics. 
Charcoal from protohist ditch $\left(49^{\circ} 31^{\prime} \mathrm{N}, 4^{\circ} 17^{\prime} \mathrm{E}\right)$. Coll and subm 1975 by B Lambot, Compiègne. Comment: date agrees with Late BronzeEarly Halstatt period, as expected.

\section{Africa}

Algeria

\section{Protohistoric Megalithic monuments series}

Human bones from megalithic monuments in N Algeria. Coll and subm 1973 by M C Chamla.

Gif-2841. Djebel Mistiri, Ain Chabro

From one of many tumuli of Djebel Mistiri $\left(35^{\circ} 25^{\prime} \mathrm{N}, 8^{\circ} \mathrm{E}\right)$.

Gif-2842. Bou Nouara, Djebel Mazela $1700 \pm 110$ E).

From largest necropolis of $\mathrm{N}$ Africa, Djebel Mazela $\left(36^{\circ} 10^{\prime} \mathrm{N}, 6^{\circ} 50^{\prime}\right.$

\section{Gif-2843. Sil}

From burial cave, Sila $\left(36^{\circ} 10^{\prime} \mathrm{N}, 6^{\circ} 45^{\prime} \mathrm{E}\right)$.

\section{Gif-2844. Tiddis}

From Berber tomb of Tiddis $\left(36^{\circ} 35^{\prime} \mathrm{N}, 6^{\circ} 35^{\prime} \mathrm{E}\right)$.

Gif-2845. Gastel

$$
1090 \pm 100
$$

Under dolmen of necropolis $\left(35^{\circ} 40^{\prime} \mathrm{N}, 8^{\circ} 10^{\prime} \mathrm{E}\right)$.

Gif-2846. Beni Messous

Under dolmen of large necropolis (300 dolmens), $\left(36^{\circ} 45^{\prime} \mathrm{N}, 3^{\circ} 1^{\prime} \mathrm{E}\right)$. General Comment: confirm expected range of ages for these cemeteries.

\section{Brezina series, $\mathbf{S}$ Algeria}

Charcoal from Neolithic sites near Brezina $\left(33^{\circ} 06^{\prime} \mathrm{N}, 1^{\circ} 15^{\prime} \mathrm{E}\right)$ Saharian Atlas. Coll and subm 1972 by L Balout, Inst Paléont Humaine, Paris.

Gif-3011. Grotte de l'Equidé, Grottes d'El Arouī

Superficial sample, $0.40 \mathrm{~m}$ depth.

$$
3600 \pm 130
$$

\section{Gif-3012. Station du Méandre, $M 1$}

$$
5350 \pm 130
$$

From hearth in lowest Level M 1. Comment: date may be compared to Gif-883: $5850 \pm 150$ (R, 1972, v 14, p 291) from same site, at foot of wall with rupestral paintings. Site settlement was probably very short.

General Comment: dates indicate that these two sites, although near each other, were not occupied at same time. 
Niger

Gif-3547. Djaba

Wood from beam of ancient building, at Djaba $\left(21^{\circ} \mathrm{N}, 12^{\circ} 30^{\prime} \mathrm{E}\right)$. Coll and subm 1975 by W Savy, Paris. Comment: dates remains of important town.

\section{Djado series}

Wood from ruins of Djado $\left(21^{\circ} \mathrm{N}, 12^{\circ} 20^{\prime} \mathrm{E}\right)$. Coll and subm 1977 by W Savy. Djado, like Djaba, is important ancient town in desert region of Ténéré.

Gif-4130. Djado, 1

$90 \pm 90$

From lintel in wall from interior of town.

Gif-4131. Djado, 4

$270 \pm 90$

\section{Ténéré series}

Samples from open air sites, in Ténéré. Coll 1972 and subm 1975 by J P Roset, ORSTOM, Fort-Lamy.

Gif-3639. Ténéré, No. 7

$3180 \pm 100$

Human bones from burial, at depth $1.50 \mathrm{~m}$, under stone blocks $\left(18^{\circ} 29^{\prime}\right.$ $\left.\mathrm{N}, 10^{\circ} 03^{\prime} \mathrm{E}\right)$.

Gif-3640. Ténéré, No. 8

Modern

Charcoal from hearth, at depth $0.20 \mathrm{~m}\left(18^{\circ} 33^{\prime} \mathrm{N}, 9^{\circ} 46^{\prime} \mathrm{E}\right)$. Comment: dates recent hearth in archaeol site.

Sudan

\section{Saï Island series}

Saï I., one of largest islands of Nile $\mathrm{R}\left(20^{\circ} 40^{\prime} \mathrm{N}, 30^{\circ} 20^{\prime} \mathrm{E}\right)$, is in important strategic position. Site was occupied from earliest antiquity until now. Samples coll 1974 by B Gratien and J Vercoutter and subm 1975 by J Vercoutter, Univ Lille.

\section{Gif-3581. Saï, SKC 3-T 37}

$3810 \pm 110$

Leather from burial of Late Kerme culture. Comment: cal 2620-1975 BC agrees well with expected age.

Gif-3582. Saï, SACJ-T 21

$4010 \pm 120$

Charcoal from cemetery of New Egyptian Empire, cal ca 1580-1200 BC. Comment: cal 2885-2310 BC might correspond to Middle Empire: cemetery was re-used in New Empire.

Gif-3583. Saï, SAFJ-C 14

$3880 \pm 120$

Carbonized wood from burial of Middle Empire. Comment: cal 2650$2130 \mathrm{BC}$, agrees well with archaeol data (Gratien, 1974; Vercoutter, 1974). 
Ethiopia

\section{Matara series}

Samples from Matara site $\left(14^{\circ} 4^{\prime} \mathrm{N}, 39^{\circ} 4^{\prime} \mathrm{E}\right)$ attributed to "Axum" period. Coll and subm 1975 by F Anfray, Inst Ethiopien Archéol, Addis Ababa.

Gif-3316. Matara, 1

$1460 \pm 90$

Charcoal.

Gif-3320. Matara, 5

$1250 \pm 90$

Carbonized barley grains.

General Comment: agrees well with archaeol data.

\section{Ethiopian Christian monuments series}

Wood from ancient churches in Ethiopia, some of which were discovered during French expedition 1971-1973. Samples coll and subm 1973 by C Lepage, CNRS, Paris.

Gif-2922. Abraha Asbeha, 1

$1290 \pm 90$

Monument A.

Gif-2923. Zarema, 2

$1560 \pm 90$

From wood piece engraved on one side, Monument B.

Gif-2924. Agobo, 3bis

$860 \pm 80$

Gif-2925. Agobo, 3

$800 \pm 90$

Monument C.

Gif-2926. Mikael Amba 4

$700 \pm 90$

From wood piece engraved on one side.

General Comment: dates precise period of building of ancient churches $(>200)$ and of use of typical architectural elements.

\section{Yeha series}

Charcoal from archaeol site of Yeha $\left(14^{\circ} 04^{\prime} \mathrm{N}, 39^{\circ} 4^{\prime} \mathrm{E}\right)$ attributed to "pre-Axum" period. Coll and subm 1975 by F Anfray.

Gif-3317. Yeha, 2

$$
2260 \pm 100
$$

Gif-3318. Yeha, 3

$2100 \pm 100$

Gif-3319. Yeha, 4

$2150 \pm 100$

General Comment: agrees well with archaeol estimate.

\section{Harrar megalithic monuments}

Charcoal from megalithic monuments of Harrar region. Two types of monuments, dolmen cists and tumuli are distinguished, according to struc- 
ture. Coll and subm 1973-1974 by R Joussaume, Lab Anthropol, Univ Rennes.

Gif-3040. Ganda Hassan-Abdi II $3200 \pm 100$

Under dolmen cist II, depth $0.60 \mathrm{~m}\left(9^{\circ} 25^{\prime} \mathrm{N}, 41^{\circ} 38^{\prime} \mathrm{E}\right)$.

\section{Gif-3039. Ganda Hassan-Abdi I}

$$
3450 \pm 100
$$

Under dolmen cist II, depth $0.80 \mathrm{~m}$.

\section{Gif-3041. Ganda Hassan-Abdi III}

$$
1170 \pm 90
$$

From refuse pit at base of thick walls of old town.

\section{Gif-3042. Ganda Hassan-Abdi V}

$$
1030 \pm 90
$$

From bone deposit, 100m from refuse pit. Comment: both Gif-3041 and -3042 confirm Medieval age of these monuments.

Gif-3288. Sourre-Kabanawa, Tchelenko, 1

$$
940 \pm 90
$$

Cist $6\left(9^{\circ} 35^{\prime} \mathrm{N}, 41^{\circ} 40^{\prime} \mathrm{E}\right)$.

Gif-3289. Sourre-Kabanawa, 2

$$
940 \pm 90
$$

From room of monument with circular chamber.

Gif-3290. Sourre-Kabanawa, 3

$$
1000 \pm 90
$$

In polypod vase, in same monument as Gif-3288.

Gif-3291. Sourre-Kabanawa, 5

$$
1150 \pm 90
$$

Beside two crania, at base of same monument, as Gif-3288.

Gif-3292. Sourre-Kabanawa, 6 $1170 \pm 90$

From tumulus.

General Comment: dates indicate that these monuments, difficult to date by archaeol assoc belong either to Neolithic or Medieval times.

Congo

\section{Grotte of Ntadi Yomba series, Niari}

Samples from rock shelter (ca $4^{\circ} 19^{\prime} \mathrm{S}, 13^{\circ} 47^{\prime} \mathrm{E}$ ). Coll by R Lanfranchi and subm 1976-78 by R de Bayle des Hermens. Assoc with Neolithic industry of Tshitolian type.

Gif-4219. Grotte of Ntadi, 1-76-NY $\mathbf{3 7 0} \pm \mathbf{8 0}$

Charcoal from upper level, depth 20 to $25 \mathrm{~cm}$.

Gif-4220. Grotte of Ntadi, 2-76-NY $\mathbf{2 7 0} \pm \mathbf{8 0}$

Charcoal from upper level, depth 50 to $55 \mathrm{~cm}$.

Gif-4221. Grotte of Ntadi, 3-76-NY $\mathbf{3 0 0} \pm \mathbf{8 0}$

Charcoal from upper level, depth 60 to $65 \mathrm{~cm}$. 
Gif-4391. Grotte of Ntadi, 1-77-NY

Charcoal from Level A.

Gif-4614. Grotte of Ntadi, 2-78-NY

$690 \pm 200$

Charcoal from Level A. Comment: undersized sample. Probably contaminated with charcoal from upper levels.

Gif-4392. Grotte of Ntadi, 2-77-NY

$7090 \pm 140$

Charcoal from base of Level B from Early Tshitolian age.

Gif-4613. Grotte of Ntadi, 1-78-NY

$6890 \pm 160$

Akatina shells from Level B, depth 98 to $100 \mathrm{~cm}$, from Early Tshitolian age.

General Comment: dates from lower levels agree well with Early Tshitolian industry found in cave (De Maret, 1982).

Gif-4223. Pointe Noire, 1-77 PN

$1240 \pm 90$

Charcoal in Late Neolithic habitat in sandy dune $\left(4^{\circ} 46^{\prime} \mathrm{S}, 11^{\circ} 53^{\prime} \mathrm{E}\right)$. Coll by R Lanfranchi and subm 1977 by R de Bayle des Hermens, Inst Paléont Humaine, Paris. Comment: in expected age range.

Madagascar

Andranosoa series

Charcoal from archaeol sites in $\mathrm{N}$ part of Androy region $\left(24^{\circ} 35^{\prime} \mathrm{S}, 45^{\circ}\right.$ $21^{\prime}$ E). Coll and subm 1978 by J P Emphoux and J A Rakotoarisoa, Mus Art \& Archaeol, Antananarivo.

Gif-4496. Beropitika $750 \pm 90$

From deepest occupation level.

Gif-4570. Andranosoa, 1 $730 \pm 90$

Assoc with abundant ceramics.

Gif-4751. Andranosoa, 2

$920 \pm 90$

From refuse pit of same site as Gif- 4570 .

General Comment: after calibrations, first settlement of Androy region is dated between X and XIV centuries.

Gif-5154. Ambohitrikanlaka

$380 \pm 90$

Charcoal in Level 3 from Site $1,13 \mathrm{~km}$ E Antananarivo $\left(18^{\circ} 52^{\prime} \mathrm{S}, 47^{\circ}\right.$ $30^{\prime}$ E). Subm 1980 by J P Domenichini, Mus Art \& Archaeol, Antananarivo. Comment: dates first settlement of this region.

\section{Fanongoavano series}

Charcoal from ancient political site $\left(19^{\circ} 03^{\prime} \mathrm{S}, 47^{\circ} 50^{\prime} \mathrm{E}\right)$. Coll and subm 1981 by $\mathrm{D}$ Rasamuel, Univ Madagascar, Antananarivo. 
Gif-5472. Fanongoavana, F 5 b

Gif-5471. Fanongoavana, E 5 m

General Comment: Gif-5471 dates first settlement in region of "Hauts Plateaux" in Madagascar.

Gif-3403. Befotaka Bay

$300 \pm 90$

Wood from stem post of boat found in Befotaka Bay $\left(13^{\circ} 11^{\prime} \mathrm{S}, 48^{\circ} 56^{\prime}\right.$ E). Coll by $\mathrm{H}$ Bruelle and subm 1974 by $\mathrm{G}$ Pain, Tananarive.

\section{Other African Countries}

Morocco

\section{Kef el Baroud series, Ben Slimane}

Charcoal from site with burial $\left(33^{\circ} 40^{\prime} \mathrm{N}, 7^{\circ} 1^{\prime} \mathrm{W}\right)$. Coll and subm 1974 by A de Wailly, Casablanca.

Gif-2888. Kel el Baroud, K B G

From hearth from gray level, around human skeleton.

Gif-2889. Kef el Baroud, K B B

$4750 \pm 110$

From white level underlying Gif-2888. Comment: slightly too young if compared to Gif-2888, but samples contained abundant rootlets.

\section{Mauritania}

Gif-3001. Tumulus Mauny, Massif R'Kiz

Human bones from grave in Mauny tumulus $\left(17^{\circ} 25^{\prime} \mathrm{N}, 10^{\circ} 25^{\prime} \mathrm{W}\right)$, assoc with iron and bronze artifacts. Coll and subm 1973 by C Richir, Bordeaux. Comment: date does not confirm expected pre-Islamic age.

Zä̈re

Gif-4158. Kinshasa

$1710 \pm 90$

Charcoal from site near Kinshasa $\left(4^{\circ} 21^{\prime} \mathrm{S}, 15^{\circ} 32^{\prime} \mathrm{E}\right)$. Coll and subm 1974 by D Cahen. Assoc with Iron Age potsherds. Comment: dates beginning of metallurgy in this part of Africa.

Gabon

Gif-4157. Ovendo

$5040 \pm 130$

Charcoal assoc with Late Stone Age microlithic industry $\left(0^{\circ} 20^{\prime} \mathrm{S}, 9^{\circ}\right.$ 28' E). Coll and subm 1978 by D Cahen, Mus Royal Afrique Centrale, Tervuren, Belgium. Comment (DC): agrees with dates previously reported for other Late Tshitolian assemblages in Congo: Gif-459-460: $4030 \pm 200$ and $3930 \pm 200$ BP (R, 1971, v 13, p 224) and in Zaïre: Lv-162: $5750 \pm 110$. 
Egypt

Gif-3355. Luxor, no. 1255

$3080 \pm 90$

Carbonized wood from tomb of Touy Queen, Valley of Queens $\left(25^{\circ}\right.$ $\left.41^{\prime} \mathrm{N}, 32^{\circ} 24^{\prime} \mathrm{E}\right)$. Coll and subm 1974 by $\mathrm{S}$ Delbourgo, Palais Louvre, Paris. Comment: Touy Queen belongs to XIXth dynasty, cal 1320-1200 BC agrees with this data.

\section{Djibouti Republic}

Gif-3321. Balho

$2830 \pm 130$

Human bones from "Galla" sepulcher of pre-Islamic and pre-Afar period, Bahlo region (ca $12^{\circ} \mathrm{N}, 42^{\circ} 10^{\prime} \mathrm{E}$ ). Coll and subm 1974 by $\mathrm{R}$ Ferry, Djibouti Republic.

TABLE 1

Canadian Arctic series

\begin{tabular}{|c|c|c|c|c|c|c|}
\hline Sample & Site & $\begin{array}{c}\text { Geog } \\
\text { coordinates }\end{array}$ & Date & Material & Culture & Age \\
\hline Gif-2967 & $\begin{array}{l}\text { Site Cordeau } \\
\text { DIA-1. House A }\end{array}$ & $\begin{array}{l}\left(60^{\circ} 51^{\prime} \mathrm{N},\right. \\
\left.69^{\circ} 52^{\prime} \mathrm{W}\right)\end{array}$ & 1973 & Charred fat & Dorset & $1170 \pm 100$ \\
\hline-2968 & $\begin{array}{l}\text { Site Cordeau } \\
\text { DIA-1. House F }\end{array}$ & $\begin{array}{l}\left(60^{\circ} 51^{\prime} \mathrm{N}\right. \\
\left.69^{\circ} 52^{\prime} \mathrm{W}\right)\end{array}$ & 1973 & Charred fat & Dorset & $1350 \pm 100$ \\
\hline-3002 & $\begin{array}{l}\text { Site DIA-4 } \\
\text { House A }\end{array}$ & $\begin{array}{l}\left(60^{\circ} 55^{\prime} \mathrm{N}\right. \\
\left.69^{\circ} 57^{\prime} \mathrm{W}\right)\end{array}$ & 1973 & Charcoal & Dorset & $470 \pm 90$ \\
\hline-3003 & $\begin{array}{l}\text { Site DIA-4 } \\
\text { Refuse pit }\end{array}$ & $\begin{array}{l}\left(60^{\circ} 55^{\prime} \mathrm{N}\right. \\
\left.69^{\circ} 57^{\prime} \mathrm{W}\right)\end{array}$ & 1973 & Charcoal & Dorset & $1080 \pm 100$ \\
\hline-3004 & $\begin{array}{l}\text { Site DIÁ-4 } \\
\text { House with passage }\end{array}$ & $\begin{array}{l}\left(60^{\circ} 55^{\prime} \mathrm{N},\right. \\
\left.69^{\circ} 57^{\prime} \mathrm{W}\right)\end{array}$ & 1973 & Charred fat & Dorset & $990 \pm 90$ \\
\hline-2970 & $\begin{array}{l}\text { Site DIA-4 } \\
\text { House } 4 \mathrm{~F}\end{array}$ & $\begin{array}{l}\left(60^{\circ} 55^{\prime} \mathrm{N},\right. \\
\left.69^{\circ} 57^{\prime} \mathrm{W}\right)\end{array}$ & 1973 & Charred fat & Dorset & $930 \pm 90$ \\
\hline-2969 & $\begin{array}{l}\text { Site DIA-25 } \\
\text { Amittug }\end{array}$ & & 1973 & Charred fat & & $1900 \pm 110$ \\
\hline-4207 & $\begin{array}{l}\text { Ariane } \\
\text { Anse du Talon } \\
\text { Site Je EL-2 }\end{array}$ & $\begin{array}{l}\left(69^{\circ} 49^{\prime} \mathrm{N},\right. \\
\left.69^{\circ} 52^{\prime} \mathrm{W}\right)\end{array}$ & 1976 & Charcoal & Dorset & $700 \pm 80$ \\
\hline-4208 & $\begin{array}{l}\text { Site DIA-4 } \\
\text { Uplifted beach }\end{array}$ & $\begin{array}{l}\left(60^{\circ} 56^{\prime} \mathrm{N},\right. \\
\left.69^{\circ} 58^{\prime} \mathrm{W}\right)\end{array}$ & 1976 & Charcoal & Dorset & $620 \pm 80$ \\
\hline-4209 & $\begin{array}{l}\text { Site DIA 10-D } \\
\text { Illutalialuk } \\
\text { Ile aux Iglous }\end{array}$ & $\begin{array}{l}\left(60^{\circ} 51^{\prime} \mathrm{N},\right. \\
\left.69^{\circ} 52^{\prime} \mathrm{W}\right)\end{array}$ & 1976 & Charcoal & Thule & $810 \pm 80$ \\
\hline-4210 & $\begin{array}{l}\text { Site DIA-1 } \\
\text { Ile du Diana }\end{array}$ & $\begin{array}{l}\left(60^{\circ} 56^{\prime} \mathrm{N},\right. \\
\left.69^{\circ} 57^{\prime} \mathrm{W}\right)\end{array}$ & 1976 & $\begin{array}{l}\text { Organic } \\
\text { matter }\end{array}$ & Dorset & $540 \pm 80$ \\
\hline-4211 & Site DIA-1 & $\begin{array}{l}\left(60^{\circ} 56^{\prime} \mathrm{N}\right. \\
\left.69^{\circ} 57^{\prime} \mathrm{W}\right)\end{array}$ & 1976 & Soil & Dorset & Modern \\
\hline-4212 & $\begin{array}{l}\text { Site DIA-10 } \\
\text { Illutalialuk } \\
\text { Ile aux Iglous }\end{array}$ & $\begin{array}{l}\left(60^{\circ} 51^{\prime} \mathrm{N},\right. \\
\left.69^{\circ} 52^{\prime} \mathrm{W}\right)\end{array}$ & 1976 & Peat & & $2090 \pm 100$ \\
\hline-4213 & $\begin{array}{l}\text { Site DIA-10 } \\
\text { Illutalialuk }\end{array}$ & $\begin{array}{l}\left(60^{\circ} 51^{\prime} \mathrm{N},\right. \\
\left.69^{\circ} 52^{\prime} \mathrm{W}\right)\end{array}$ & 1976 & Soil & & Modern \\
\hline-3500 & $\begin{array}{l}\text { Heel Cove } \\
\text { VHC 475-480 }\end{array}$ & $\begin{array}{l}\left(60^{\circ} 47^{\prime} \mathrm{N}\right. \\
\left.69^{\circ} 51^{\prime} \mathrm{W}\right)\end{array}$ & 1973 & $\begin{array}{l}\text { Lacustrine } \\
\text { sediment }\end{array}$ & & $6460 \pm 160$ \\
\hline
\end{tabular}


North America

Canada

\section{Canadian Arctic series}

Samples mainly from Dorset houses around Diana Bay and on Diana I., New Quebec were coll and subm by P Plumet, Univ Quebec, Montreal. These new results (see Table 1) complete data already pub (R, 1974, v 16, p 47) for same region.

General Comment: settlement of Ungava Bay by Paleo-Eskimos occurred late, at Dorset period, which lasted until end of 15 th century AD, although Thule people had already arrived in the area (Plumet, 1977, 1982). PaleoEskimos settlement on Diana I. occurred after Thule people had occupied region for one century: $1480 \pm 90 \mathrm{BP}$ (Gif-3002).

\section{Mesoamerica}

Guatemala

\section{Pueblo Viejo Chichaj series}

Samples from Pueblo Viejo Chichaj site $\left(15^{\circ} 07^{\prime} \mathrm{N}, 90^{\circ} 49^{\prime}\right.$ W). Coll 1974 by A Ichon and subm by H Lehmann, CNRS, Paris.

Gif-3895. Pyramid GP-2

Carbonized wood from fallen roof of temple.

Gif-3896. Pyramid GP-I

$110 \pm 90$

Similar to Gif-3895.

Gif-3897. Structure C-1

Bones, from funeral urn.

General Comment (AI): site attributed to 15 th century and assumed deserted since Spanish conquest; dates disagree with this assumption.

\section{Los Cimientos Chustum series}

Charcoal from Los Cimientos Chustum site $\left(15^{\circ} 13^{\prime} \mathrm{N}, 90^{\circ} 52^{\prime} \mathrm{W}\right)$. Coll 1974 by A Ichon and subm by H Lehmann.

Gif-3601. Los Cimientos I, Terrace B $790 \pm 90$

From Sepulture 2 of important chief.

Gif-3894. Los Cimientos 2, Terrace B

Modern

From fill of water vat, in Structure 12.

Gif-3602. Los Cimientos 1, Terrace T

$840 \pm 90$

From hearth, in Structure 4.

General Comment: Gif-3601 and -3602 date first occupation of site very well, at beginning of Postclassic period, but Gif-3894 disagrees with expected age. 
Los Cerritos Chijoj Canilla Quiche series

Charcoal from ceremonial structures at Los Cerritos Chijoj site $\left(15^{\circ}\right.$ $10^{\prime} \mathrm{N}, 90^{\circ} 50^{\prime} \mathrm{W}$ ). Coll by A Ichon and subm 1974-1975 by H Lehmann.

Gif-3242. Los Cerritos, Structure 1, Excavation T 3, Level 100

$1630 \pm 70$

Gif-3243. Los Cerritos, Structure 1, tomb

$1640 \pm 70$

Gif-3244. Los Cerritos, Structure 1, Excavation T 3, Level 250

$$
1620 \pm 70
$$

Gif-3607. Los Cerritos, Group A, Structure 1

$1360 \pm 100$

Gif-3603. Los Cerritos, Group A, Structure 10

$1480 \pm 90$

Comment: cal AD 345-645 does not agree well with polychrome potsherds of assoc Late Classic period (cal ca AD 600-900).

Gif-3604. Los Cerritos, Group A, Structure 8 $1400 \pm 100$

Gif-3605. Los Cerritos, Group A, Structure $10 \quad 1540 \pm 100$ period.

From Tomb 1. Comment: dates re-use of Tomb 1 at Late Postclassic

Gif-3606. Los Cerritos, Group A, Structure 7

$1650 \pm 100$

Charcoal from center of platform.

Gif-3246. Los Cerritos, Group A, Structure 3, Level $201590 \pm 70$

Gif-3248. Los Cerritos, Group A, Structure 4, Level $401590 \pm 70$

Gif-3250. Los Cerritos, Group A, Structure 4, Level $501370 \pm 70$

Gif-3251. Los Cerritos, Group A, Structure 4, Level $0 \quad 950 \pm 80$

Comment: dates end of occupation of site.

Gif-3892. Los Cerritos, Group A, Structure 5

$1340 \pm 100$

Gif-3239. Chijoj, Mound 1

$1400 \pm 70$

Gif-3247. Chijoj, Mound 1, base Level 120

$1830 \pm 80$

General Comment: site was occupied at different times. Protoclassic occupation, cal AD 100-300, was not very important; main occupation was during Late Classic period, with building of Group A and polychrome ceramics, cal AD 600-900. Some structures were re-used at Epiclassic, cal AD 900-100, and probably at Postclassic periods.

Mexico

Tonina series, Chiapas

Charcoal from Classic Maya City $\left(16^{\circ} 50^{\prime} \mathrm{N}, 92^{\circ} \mathrm{W}\right)$. Coll and subm 1971-1980 by P Becquelin, Mus Homme, Paris. 
Gif-3252. Tonina, 128

$1160 \pm 60$

Assoc with potsherds attributed to Late Classic period. Comment: agrees well with expected age.

Gif-3253. Tonina, 619

$1340 \pm 60$

From building level of group of habitations.

Gif-3254. Tonina, 659

$1350 \pm 60$

From tomb assoc with group of habitations dated by Gif-3253.

Gif-3255. Tonina, 831

$1380 \pm 60$

From occupation level. Comment: agrees well with expected age.

Gif-5056. Tonina, 928-942

$260 \pm 60$

From hearth in group of habitations. Comment: corresponds to recent occupation of site.

Gif-5057. Tonina, 1132

$980 \pm 80$

From depth $1.73 \mathrm{~m}$ in occupation level of group of habitations; F 3 attributed to beginning of Late Classic period. Comment: date is a little younger than expected.

Gif-5058. Tonina, 1509

$1300 \pm 60$

Piece of wood from frame inside stucco block.

Gif-5059. Tonina, 1253

$1310 \pm 60$

From depth $1.90 \mathrm{~m}$ in occupation level, S E part.

Gif-5060. Tonina, 1388

$1950 \pm 60$

From depth 1.00 to $1.20 \mathrm{~m}$, in ashy soil. Comment: dates early occupation of site.

Gif-5359. Tonina, 1845

$910 \pm 80$

From level attributed to Early Postclassic period. Comment: date agrees with expected age.

Gif-5360. Chix, Tonina, 1663

$2070 \pm 120$

From occupation level attributed to Late Preclassic period, from site near Tonina.

Gif-5362. Chix, Tonina, 1666

$1720 \pm 100$

From same occupation level as Gif-5360.

Gif-5361. Chix, Tonina, 1664

$1840 \pm 90$

From same occupation level as Gif-5360.

Gif-5364. Rancho de Santa Rosa, Tonina 1964

$1930 \pm 120$

From occupation level from Late Preclassic site, near Tonina. 
General Comment: dates establish chronology of occupation from Preclassic to Postclassic periods for that region.

\section{Bahia de Kino series}

Charcoal from human settlements on littoral sandbar of Holocene transgression $\left(28^{\circ} 50^{\prime} \mathrm{N}, 114^{\circ} \mathrm{E}\right)$. Coll and subm 1975 by $\mathrm{N}$ Petit-Maire, CNRS, Marseille.

$$
\begin{array}{llr}
\text { Gif-3683. } & \text { Bahia de Kino, K IV, }-5 \mathrm{~cm} & 270 \pm 90 \\
\text { Gif-3685. } & \text { Bahia de Kino, K XI, }-10 \mathrm{~cm} & 1290 \pm 90 \\
\text { Gif-3687. } & \text { Bahia de Kino, K IX, }-15 \mathrm{~cm} & 300 \pm 90 \\
\text { Gif-3690. } & \text { Bahia de Kino, K IX, }-60 \mathrm{~cm} & 310 \pm 90 \\
\text { Gif-4055. } & \text { Playa San Bartolo, Sonora } & \mathbf{7 6 3 0} \pm \mathbf{4 6 0}
\end{array}
$$

Bones of large mammals (Camelidae) in paleolacustrine deposit $\left(29^{\circ} \mathrm{N}\right.$, $\left.114^{\circ} \mathrm{S}\right)$. Coll and subm 1976 by N Petit-Maire. Comment: date proves late existence of camel in Mexico (Petit-Marie \& Casta, 1977).

\section{South America}

Peru

Gif-3564. Huazmey Valley, Peru, HN 1

$3750 \pm 110$

Carbonized wood from preceramic open-air site $\left(10^{\circ} 03^{\prime} \mathrm{S}, 78^{\circ} 10^{\prime} \mathrm{W}\right)$. Depth, $1.50 \mathrm{~m}$. Coll by D Bonavia and subm 1975 by C Chauchat. Comment: dates Late Peruvian preceramic site.

\section{Paijan complex series, Pampa de Cupisnique}

"Paijan" complex is $50 \mathrm{~km}$ from Paijan in Moche Valley, S side of Pampa de Cupisnique. Site is very large and complex group of lithic surface scatters in desert. Twofold division is distinguished between numerous discovered sites (Chauchat, 1976, 1982). Samples coll by S Uceda and C Chauchat and subm 1974, 1976, 1981 by C Chauchat, Univ Bordeaux (see Table 2).

General Comment (CC): ages of shells seem systematically younger than ages of charcoal at this site. If statistical margin for charcoal is doubled for each date, Paijanian lasted from 10,720 to $7940 \mathrm{BP}$, which means $3000 \mathrm{yr}$, at Cupisnique. These results may be compared to other dates obtained for Paijanian, for Quirihuac rock shelter, in Moche Valley; oldest one is 12,795 \pm 350 (Gx-2021) and for Cerro Chivateros: 10,430 \pm 160 (UCLA-683). Dated bones (Gif-4116) are ribs from whole skeleton of Mylodontide, Scelidodon sp (id by R Hoffstetter). Date corresponds either to Late Paijanian period and proves existence of this fauna at that time or to contamination of bones (Chauchat, 1982). Gif-3565 is not significant: sample is too superficial. 
TABLE 2

Pampa de Cupisnique

\begin{tabular}{|c|c|c|c|c|c|}
\hline Sample & Loc & $\begin{array}{c}\text { Geog } \\
\text { coordinates }\end{array}$ & Ref & Material & Age \\
\hline Gif-3565 & Camp & $\begin{array}{l}\left(7^{\circ} 34^{\prime} \mathrm{S},\right. \\
\left.79^{\circ} 20^{\prime} \mathrm{W}\right)\end{array}$ & $12-\mathrm{U} 7-\mathrm{C}$ & Charcoal & $5490 \pm 140$ \\
\hline-4165 & Camp & $\begin{array}{l}\left(7^{\circ} 34^{\prime} \mathrm{S},\right. \\
\left.79^{\circ} 20^{\prime} \mathrm{W}\right)\end{array}$ & $12-U 7-S$ & Snail shells & $8810 \pm 160$ \\
\hline-4161 & Camp & $\begin{array}{l}\left(7^{\circ} 34^{\prime} \mathrm{S},\right. \\
\left.79^{\circ} 20^{\prime} \mathrm{W}\right)\end{array}$ & 13-UI-C & Charcoal & $9810 \pm 180$ \\
\hline-4163 & Camp & $\begin{array}{l}\left(7^{\circ} 34^{\prime} \mathrm{S},\right. \\
\left.79^{\circ} 20^{\prime} \mathrm{W}\right)\end{array}$ & 13-U I-S & Snail shells & $7740 \pm 150$ \\
\hline-3781 & Tomb T 2 & $\begin{array}{l}\left(7^{\circ} 34^{\prime} \mathrm{S},\right. \\
\left.79^{\circ} 20^{\prime} \mathrm{W}\right)\end{array}$ & 13-U2-T2 & Charcoal & $10,200 \pm 180$ \\
\hline-4914 & Quarry & $\begin{array}{l}\left(7^{\circ} 34^{\prime} \mathrm{S}\right. \\
\left.79^{\circ} 20^{\prime} \mathrm{W}\right)\end{array}$ & 13-U II & Charcoal & $9490 \pm 170$ \\
\hline-4915 & Camp & $\begin{array}{l}\left(7^{\circ} 34^{\prime} \mathrm{S},\right. \\
\left.79^{\circ} 20^{\prime} \mathrm{W}\right)\end{array}$ & 13-U29 & Charcoal & $9300 \pm 170$ \\
\hline-4162 & Camp & $\begin{array}{l}\left(7^{\circ} 33^{\prime} \mathrm{S}\right. \\
\left.79^{\circ} 19^{\prime} \mathrm{W}\right)\end{array}$ & 27-U I & Charcoal & $8260 \pm 170$ \\
\hline-5159 & Camp & $\begin{array}{l}\left(7^{\circ} 33^{\prime} \mathrm{S},\right. \\
\left.79^{\circ} 19^{\prime} \mathrm{W}\right)\end{array}$ & 14-U2-BH & Charcoal & $8730 \pm 160$ \\
\hline-5160 & Camp & $\begin{array}{l}\left(7^{\circ} 33^{\prime} \mathrm{S}\right. \\
\left.79^{\circ} 19^{\prime} \mathrm{W}\right)\end{array}$ & 14-U2-BD & Charcoal & $10,380 \pm 170$ \\
\hline-5161 & Camp & $\begin{array}{l}\left(7^{\circ} 33^{\prime} \mathrm{S},\right. \\
\left.79^{\circ} 19^{\prime} \mathrm{W}\right)\end{array}$ & 14-U2-BG & Charcoal & $9360 \pm 170$ \\
\hline-5162 & Camp & $\begin{array}{l}\left(7^{\circ} 33^{\prime} \mathrm{S},\right. \\
\left.79^{\circ} 19^{\prime} \mathrm{W}\right)\end{array}$ & 14-U2-BI & Charcoal & $9600 \pm 170$ \\
\hline-4116 & Paleontol site & $\begin{array}{l}\left(7^{\circ} 32^{\prime} \mathrm{S},\right. \\
\left.79^{\circ} 19^{\prime} \mathrm{W}\right)\end{array}$ & & Scelidodon & $8910 \pm 200$ \\
\hline-4912 & Camp, refuse pit & $\begin{array}{l}\left(7^{\circ} 39^{\prime} \mathrm{S},\right. \\
\left.79^{\circ} 05^{\prime} \mathrm{W}\right)\end{array}$ & 5-U4-C & Charcoal & $9670 \pm 170$ \\
\hline-4913 & Camp, refuse pit & $\begin{array}{l}\left(7^{\circ} 39^{\prime} \mathrm{S}\right. \\
\left.79^{\circ} 05^{\prime} \mathrm{W}\right)\end{array}$ & 5-U4-S & Snail shells & $9510 \pm 170$ \\
\hline-4164 & Camp & $\begin{array}{l}\left(7^{\circ} 39^{\prime} \mathrm{S}\right. \\
\left.79^{\circ} 05^{\prime} \mathrm{W}\right)\end{array}$ & 12-U3 & Snail shells & $7220 \pm 140$ \\
\hline
\end{tabular}

Brazil

Gif-5365. Aldeia Tuatuari, Mato Grosso

$680 \pm 60$

Charcoal from site $\left(12^{\circ} 15^{\prime} \mathrm{S}, 53^{\circ} 20^{\prime} \mathrm{W}\right)$ attributed to Ipavu phase. Coll and subm 1980 by $\mathrm{P}$ Becquelin, Mus Homme, Paris. Comment: date agrees with expected range of dating.

Gif-3307. Lagoa das Onças, Haut Xingu

$600 \pm 80$

Carbonized wood from site near lake where, according to Amazonian cultural traditions, many ritual objects were dropped $\left(12^{\circ} 07^{\prime} \mathrm{S}, 53^{\circ} 24^{\prime} \mathrm{W}\right)$. Coll and subm 1973 by $\mathrm{P}$ Becquelin. Comment: date agrees well with expected age.

\section{Argentina}

\section{Las Lomas series, Santiago del Estero}

Charcoal from Sunchituyoj site and Averias tradition (ca $29^{\circ} 30^{\prime} \mathrm{S}, 61^{\circ}$ $30^{\prime}$ W). Coll and subm 1974 by A M Lorandi, Fac Ciencias Nat y Mus, La Plata, Argentina. 
Gif-3365. Las Lomas, B 5, Sec 1, Level 3

Gif-3366. Las Lomas, B 4, 1, 40-60cm

Gif-3367. Las Lomas, C 5, 1, 40-60cm

Other Countries

Gif-3394. Vastogirardi, Molise, Abruzzi, Italy

$2050 \pm 90$

Charcoal from habitation site $\left(41^{\circ} 45^{\prime} \mathrm{N}, 14^{\circ} 15^{\prime} \mathrm{E}\right)$, alt $1150 \mathrm{~m}$. Coll and subm 1974 by J P Morel and M Hano, Ecole Française de Rome. Comment: data agrees with age expected from assoc ceramics.

Tureng Tepe series, Iran

Samples from tell at Tureng Tepe $\left(36^{\circ} 55^{\prime} \mathrm{N}, 54^{\circ} 35^{\prime} \mathrm{E}\right)$. Coll and subm 1974 by J Deshayes, Univ Paris I.

Gif-3340. Tureng Tepe, TTC 73-5

$1370 \pm 90$

Carbonized wood from destruction of Sassamide fortress.

Gif-3341. Tureng Tepe, TTC 73-1

$1400 \pm 90$

Carbonized wood from 1st Islamic occupation level.

Gif-3338. Tureng Tepe, TTC 69-2

$3750 \pm 230$

Carbonized wood from transition level between III C 1 and III C 2 periods.

Gif-3339. Tureng Tepe, TTC 73-6

$4000 \pm 110$

Piece of carbonized beam from terrace without archaeol assoc. Comment: dates largest structure at Tureng Tepe.

General Comment: Gif-3340 agrees with Sassanid Empire history, but Gif3341 seems too early relative to Gif-3340. Gif-3338 and -3339 confirm dates previously obtained for that site (R, 1970, v 12, p 441).

\section{Rondane series, Norway}

Charcoal and wood from typical rounded pits used by reindeer hunters. Coll and subm 1973 by E K Barth, Oslo Univ.

Gif-2801. Lille Ula, P 3 c-72

$370 \pm 80$

$\left(61^{\circ} 51^{\prime} \mathrm{N}, 9^{\circ} 40^{\prime} \mathrm{E}\right)$.

Gif-2802. Verkelsdalen, P 7-71

$430 \pm 80$

$\left(61^{\circ} 56^{\prime} \mathrm{N}, 9^{\circ} 41^{\prime} \mathrm{E}\right)$.

Iles de la Société series, French Polynesia

Charcoal from archaeol sites from Iles de la Société. Coll by B Gérard and subm 1973 by J Garanger, ORSTOM, Paris.

Gif-2830. Raiatea, R M 9-1-72

$220 \pm 80$

From site with lithic industry $\left(16^{\circ} 46^{\prime} \mathrm{S}, 151^{\circ} 25^{\prime} \mathrm{W}\right)$. 
Gif-2831. Tahiti, T M 4 2-71

From surface site $\left(17^{\circ} 30^{\prime} \mathrm{S}, 149^{\circ} 30^{\prime} \mathrm{W}\right)$. Comment: agrees with date: $\mathrm{AD}$ $1695 \pm 95(\mathrm{Gx}-1271)$ obtained for similar site in Tahiti.

General Comment: dates confirm that human settlement in Iles de la Société is recent.

\section{Gif-3541. Ngando Beach, Ile des Pins,} New Caledonia

$1980 \pm 90$

Shells in beach rock with gray coral stones, perodotite river pebbles and encrusted iron oxide stones, on Ngando Beach $\left(22^{\circ} 38^{\prime} \mathrm{S}, 167^{\circ} 33^{\prime} \mathrm{E}\right)$. Coll and subm 1975 by M J Dubois, CNRS, Paris. Comment (MJD): Ile des Pins is coralliferous Pleistocene fms, consequently, stones and pebbles scattered on beach $300 \mathrm{~m}$ long and encrusted in beach rock were brought by man. They look like projectile points fallen during fighting. This proves island was inhabited $1980 \mathrm{yr}$ ago.

\section{GEOLOGIC SAMPLES}

Sea Level Variations

Gif-2667. Knockagoneen Cliff, D, Ireland

$3210 \pm 110$

Oyster and Littorina shell bed, $+5 \pm 1 \mathrm{~m}$ above msl $\left(51^{\circ} 16^{\prime} \mathrm{N}, 9^{\circ} 03^{\prime}\right.$ W). Coll by R Keary, Dept Geol, Univ College, Galway, Ireland and subm 1972 by J Labeyrie, CFR, Gif-sur-Yvette.

Gif-2668. Sea Weed Point, Galway Bay, F, Ireland $2560 \pm 110$

Oyster shells from large oyster bed, $+15 \mathrm{~m}$ above msl $\left(51^{\circ} 15^{\prime} \mathrm{N}, 9^{\circ} 02^{\prime}\right.$ W). Coll by R Keary and subm 1972 by J Labeyrie. Comment: unexpected date for sea level $+15 \mathrm{~m}$ above $\mathrm{msl}$.

Gif-2806. Culleenamore Strandhill, Slico Bay, Ireland $3730 \pm 130$

Oyster shells from very large oyster shell reef, 1.5 to $2 \mathrm{~m}$ thick, covered by blown sand $+2 \mathrm{~m}$ above msl $\left(54^{\circ} 15^{\prime} \mathrm{N}, 8^{\circ} 30^{\prime} \mathrm{W}\right)$. Coll and subm 1973 by R Keary.

Gif-2807. Doona, Bally Croy, Mayo, B, Ireland

$5490 \pm 140$

Wood from tree trunk in peat bog, on shore, $-2 \mathrm{~m}$ below $\mathrm{msl}\left(54^{\circ} 02^{\prime}\right.$ N, $9^{\circ} 49^{\prime} \mathrm{W}$ ). Coll and subm by R Keary.

General Comment: samples date variations of Holocene sea level.

Gif-2903. Mont-Saint-Michel, France

$3440 \pm 110$

Wood from timber, from fishing equipment remains, removed by sea from sand, in Mont-Saint-Michel bay $\left(48^{\circ} 38^{\prime} \mathrm{N}, 1^{\circ} 30^{\prime} \mathrm{W}\right)$. Coll and subm 1973 by A L Homer, BRGM, Orléans. Comment: indicates that sea level was higher than today.

\section{Spanish Mediterranean coast series}

Samples from ancient shorelines, from $+2 \mathrm{~m}$ to $+7 \mathrm{~m}$ above msl, along Alboran and W Mediterranean Seas, S Spain. Most of these fossil marine 
fms belong to last Thyrrenian transgression and have been dated by $\mathrm{U} / \mathrm{Th}$ method between 114,000 and 125,000 yr BP. Others were expected to be Holocene and were dated by ${ }^{14} \mathrm{C}$. Coll and subm $1973-75$ by M Gigout, Lab Geol, Univ Orléans, as part of general study of neotectonic in Mediterranean area.

Gif-3730. Campo de Dalias, W Almeria, JFC 62-62 $4520 \pm 130$

Shells (Cardium, Pectunculus sp) ( $\left.36^{\circ} 50^{\prime} \mathrm{N}, 2^{\circ} 26^{\prime} \mathrm{W}\right)$, alt $+2 \mathrm{~m}$ above msl. Comment: corresponds to Holocene sea level.

Gif-3087. Cabo de Gata, Torre Garcia, GIG 3200

Shells (Strombus bubonius, Pectunculus sp) $\left(36^{\circ} 45^{\prime} \mathrm{N}, 2^{\circ} 15^{\prime} \mathrm{W}\right)$, alt $+6.5-7 \mathrm{~m}$ above $\mathrm{msl}$.

Gif-3088. Cabo de Huertas, Alicante, GIG $2006 \quad 10,590 \pm 190$

Shells (Strombus bubonius sp) in sandstone $\left(38^{\circ} 21^{\prime} \mathrm{N}, 0^{\circ} 29^{\prime} \mathrm{W}\right)$. Comment: presence of Strombus characterizes Thyrrenian transgression. Date is aberrant because of important recent contamination of shells, in spite of numerous mechanical and chemical treatments to eliminate calcareous encrusting.

Gif-3728. Cala Judios, Alicante, GIG 3700

Shells $\left(38^{\circ} 21^{\prime} \mathrm{N}, 0^{\circ} 29^{\prime} \mathrm{W}\right)$.

Gif-3729. Cala Judios, Alicante, GIG 3701

Shells, from another outcrop than Gif-3728.

Gif-3473. Adra region, Andalusia, JFB 12-2

$860 \pm 90$

Shells (Glycymeris sp) $\left(36^{\circ} 45^{\prime} \mathrm{N}, 3^{\circ} 01^{\prime} \mathrm{W}\right)$. Comment: dated level is not Flandrian as expected, but results of very recent shelly deposit.

Gif-3491. Adra, Andalusia, JFB 233

Shells (Thaïs, Sponclyle, Natica, Columbella sp) in littoral conglomerate, Adra, alt $+2 \mathrm{~m} \mathrm{msl}$.

Gif-3089. Torre Calahouda, Malaga, KDG 14

$390 \pm 80$

Shells (marine Lamellibranchia, Gasteropoda sp) ( $\left.36^{\circ} 29^{\prime} \mathrm{N}, 1^{\circ} 01^{\prime} \mathrm{W}\right)$, alt $+2 \mathrm{~m}$ msl. Comment: dated level is not Flandrian as expected, but results of very recent shelly deposit.

General Comment: characterization by alt, fauna, and dating of ancient littorals allows identification of same levels along coasts as well as tectonic deformations. Data from shore neotectonic indicate that deformations are more important in E Mediterranean than in W Mediterranean. Uplifts and lowerings were found to be slight and localized (Angelier et al, 1976).

Gif-2902. Ifni, Morocco

$>35,000$

Mussel shells from marine terrace, $+2 \mathrm{~m}$ alt $\left(29^{\circ} 39^{\prime}, 10^{\circ} \mathrm{W}\right)$. Coll 1973 by P Oliva and subm by $\mathrm{G}$ Baudet, Univ $\mathrm{P}$ Valery, Montpellier. Comment: shelly level corresponds to ancient high sea level, probably ca 120,000 BP. 
Gif-4542. Moulay Bou Selham lagoon, Kenitra, Morocco $3490 \pm 100$

Shelly level, 0.60 to $0.67 \mathrm{~m}$ deep, in core 7800 . Coll and subm 1978 by J Moyes, Univ Bordeaux I. Comment: dates lagoon closing that probably results from sea regression.

\section{Côte d'Ivoire series, Africa}

Carbonate samples coll and subm 1975 by R Pomel, Univ ClermontFerrand, to date recent variation of sea level of Ivory Coast. Alts are related to msl.

General Comment: these dates (Table 3) demonstrate, with previous Gif measurements (R, 1982, v 24, p 306), minor marine fluctuations since $4000 \mathrm{BP}$, with max transgression $+2 \mathrm{~m}$, ca $2000 \mathrm{BP}$, on Ivory Coast (Pomel, 1979).

\section{Gif-2914. Lopez Cape, Gabon}

$1230 \pm 90$

Shells from recent littoral sand bar $\left(0^{\circ} 39^{\prime} \mathrm{S}, 8^{\circ} 42^{\prime} \mathrm{E}\right)$. Coll and subm 1973 by J Le Fournier, Elf Soc, Boussens. Comment: dated in order to study recent evolution of sea shore and of sedimentation on that coast.

Gif-3270. Goubbhat, Djibouti Republic, 802

$5980 \pm 120$

Lamellibranch shells from conglomerate, $+4 \mathrm{~m}$ above present $\mathrm{msl}, \mathrm{SE}$ Goubbhat $\left(11^{\circ} 35^{\prime} \mathrm{N}, 42^{\circ} 44^{\prime} \mathrm{E}\right)$. Coll and subm 1974 by H Faure, CNRS, Marseille. Comment: dates max Holocene sea level.

Gif-3271. Sanda, Djibouti Republic, 818

$4630 \pm 110$

Lamellibranch shells from shelly sandy level, $+4 \mathrm{~m}$ above present msl, N Sanda $\left(11^{\circ} 37^{\prime} \mathrm{N}, 42^{\circ} 44^{\prime} \mathrm{E}\right)$. Coll and subm 1974 by H Faure. Comment: dates max Holocene sea level.

Gif-3272. Obock-Alayla, Djibouti Republic, 851

Shell (Tridacna $\mathrm{sp}$ ) from coral terrace, $+5 \mathrm{~m}$ above present msl, E Obock $\left(12^{\circ} \mathrm{N}, 43^{\circ} 18^{\prime} \mathrm{E}\right)$. Coll and subm 1974 by H Faure. Comment: corresponds to high sea level preceding Holocene high level.

TABLE 3

Cote d'Ivoire

\begin{tabular}{rlllll}
\hline Sample & \multicolumn{1}{c}{ Site } & $\begin{array}{c}\text { Geog } \\
\text { coordinates }\end{array}$ & $\begin{array}{c}\text { Alt } \\
(\mathrm{m})\end{array}$ & \multicolumn{1}{c}{ Material } & \multicolumn{1}{c}{$\begin{array}{c}\text { Age } \\
(\mathrm{yr}) \mathrm{BP}\end{array}$} \\
\hline Gif-3633 & Cap of Bereby & $\left(4^{\circ} 38^{\prime} \mathrm{N}\right.$, & +3 & Concretion & Modern \\
-3673 & Falaise, Bassa & $\begin{array}{c}\left.6^{\circ} 54^{\circ} \mathrm{W}\right) \\
4^{\circ} 37^{\prime} \mathrm{N},\end{array}$ & +0.25 & Vermetidae & Modern \\
-3674 & N'Gaty & $\begin{array}{c}\left.6^{\circ} 56^{\circ} \mathrm{W}\right) \\
\left.4^{\circ} \mathrm{N} \mathrm{N}, \mathrm{\prime}, \mathrm{W}\right)\end{array}$ & +1.50 & Falun & $1310 \pm 80$ \\
-3672 & Cap Est-Niega & $\begin{array}{c}\left(4^{\circ} 53^{\prime} \mathrm{N},\right. \\
\left.6^{\circ} 11^{\prime} \mathrm{W}\right)\end{array}$ & -1 & Conglomerate & $1480 \pm 90$ \\
-3632 & Grand-Bereby & $\begin{array}{c}\left(4^{\circ} 38^{\prime} \mathrm{N},\right. \\
\left.6^{\circ} 55^{\prime} \mathrm{W}\right)\end{array}$ & +2 & Shell & $1670 \pm 100$ \\
-3671 & Bassa & $\left(4^{\circ} 37^{\prime} \mathrm{N}\right.$, \\
$\left.6^{\circ} 55^{\prime} \mathrm{W}\right)$ & -0.50 & Conglomerate & $3790 \pm 110$ \\
\hline
\end{tabular}




\section{La Plata-Magdalena series, Argentina}

Shell from coastal region near La Plata-Magdalena, prov Buenos Aires. Coll and subm 1975 by C R Cortelezzi, Fac Sci Nat, La Plata. Alt is given in $\mathrm{m}$ above $\mathrm{msl}$.

Gif-3717. Paso de Piedra, Magdalena

$\left(34^{\circ} 50^{\prime} \mathrm{S}, 57^{\circ} 50^{\prime} \mathrm{W}\right)$, alt $3.1 \mathrm{~m}$.

Gif-3719. Canta Landa, Magdalena

$\left(35^{\circ} 02^{\prime} \mathrm{S}, 57^{\circ} 44^{\prime} \mathrm{W}\right)$, alt $4.3 \mathrm{~m}$

Gif-3722. Luis Chico, Magdalena

$\left(35^{\circ} 20^{\prime} \mathrm{S}, 57^{\circ} 08^{\prime} \mathrm{W}\right)$, alt $6 \mathrm{~m}$.

Gif-3721. Punta Piedras, Magdalena

$\left(35^{\circ} 22^{\prime} \mathrm{S}, 58^{\circ} 07^{\prime} \mathrm{W}\right)$, alt $6.5 \mathrm{~m}$.

Gif-3718. Arroyo El Pescado, La Plata

$\left(34^{\circ} 50^{\prime} \mathrm{S}, 57^{\circ} 50^{\prime} \mathrm{W}\right)$, alt $3.1 \mathrm{~m}$.

Gif-3720. Calle 105 y 126 , La Plata

$\left(34^{\circ} 50^{\prime} \mathrm{S}, 57^{\circ} 50^{\prime} \mathrm{W}\right)$, alt $3.0 \mathrm{~m}$.

$6160 \pm 150$

General Comment: dates distinguish Holocene from Tyrrhenian levels.

\section{Sao Paulo coastal plain series, Brazil}

Holocene sea-level fluctuations along State of Sao Paulo coast have been retraced by studying position and age of Sambaquis and of geol fms. Samples (Table 4) were coll and subm 1974-1976 by L Martin, ORSTOM, Observatorio Nac, Rio de Janeiro, Brazil.

General Comment: uncontestable geol records indicating Holocene sea levels were higher than at present are observed along State of Sao Paulo coastal plain. During past $6000 \mathrm{yr}$, relative sea level was subjected to 2 max ca 5000 and $3300 \mathrm{yr}$ BP and to a min ca $3800 \mathrm{yr}$ BP. Comparison between curves of sea-level variations established for several parts along this coastal plain showed that max have different amplitudes. Vertical deformation of geoïd surface furnishes best explanation of amplitude differences (Martin et al, 1979-1980).

\section{Maroni estuary series, French Guyana}

Organic sediments from level with tree trunks and leaf remains, overlain by blue marine clay, $1.10 \mathrm{~m}$ thick on bank of Maroni estuary (ca $5^{\circ} 30^{\prime}$ $\mathrm{N}, 54^{\circ} 30^{\prime} \mathrm{W}$ ). Coll and subm 1975 by G R Sieffermann, ORSTOM, Paris.

\section{Gif-3695. Maroni estuary, BSP 54}

$4260 \pm 120$ clay.

100 to $120 \mathrm{~cm}$ under present soil at top of organic level, in contact with 
TABLE 4

Sao Paulo State Coastal Plain

\begin{tabular}{|c|c|c|c|c|c|c|}
\hline Sample & Loc & $\begin{array}{c}\text { Geog } \\
\text { coordinates }\end{array}$ & Material & $\begin{array}{l}\text { Former } \\
\text { sea level } \\
(\mathrm{m})\end{array}$ & Ref & $\begin{array}{c}\text { Age } \\
\text { (yr BP) }\end{array}$ \\
\hline \multicolumn{7}{|c|}{ Samples from Sambaquis } \\
\hline Gif-3435 & Cananeia I. & $\begin{array}{l}\left(25^{\circ} 01^{\prime} \mathrm{S}\right. \\
\left.48^{\circ} 03^{\prime} \mathrm{W}\right)\end{array}$ & Oyster shell & $\begin{array}{l}\text { Above present } \\
\text { level }\end{array}$ & A 3 & $4340 \pm 100$ \\
\hline-3436 & Comprida I. & $\begin{array}{l}\left(24^{\circ} 59^{\prime} \mathrm{S}\right. \\
\left.47^{\circ} 53^{\prime} \mathrm{W}\right)\end{array}$ & Oyster shell & Below $+2 m$ & A 16 & $4120 \pm 100$ \\
\hline-3437 & Ararapiza Channel & $\begin{array}{l}\left(25^{\circ} 08^{\prime} \mathrm{S}\right. \\
\left.48^{\circ} 02^{\prime} \mathrm{W}\right)\end{array}$ & Oyster shell & $\begin{array}{l}\text { About present } \\
\text { level }\end{array}$ & A 29 & $3790 \pm 110$ \\
\hline-3641 & Sao Paulo & $\begin{array}{l}\left(24^{\circ} 30^{\prime} \mathrm{S}\right. \\
\left.47^{\circ} 27^{\prime} \mathrm{W}\right)\end{array}$ & Oyster shell & Near max & A 121 & $4750 \pm 100$ \\
\hline-3646 & Sao Paulo & $\begin{array}{l}\left(24^{\circ} 27^{\prime} \mathrm{S}\right. \\
\left.47^{\circ} 13^{\prime} \mathrm{W}\right)\end{array}$ & Oyster shell & $\begin{array}{l}\text { Above present } \\
\text { level }\end{array}$ & A 175 & $4560 \pm 110$ \\
\hline-3643 & Sao Paulo & $\begin{array}{l}\left(25^{\circ} 01^{\prime} \mathrm{S}\right. \\
\left.\quad 48^{\circ} 02^{\prime} \mathrm{W}\right)\end{array}$ & Shell & Below $+0.5 \mathrm{~m}$ & A 81 & $1850 \pm 100$ \\
\hline-3645 & Sao Paulo & $\begin{array}{c}\left(24^{\circ} 58^{\prime} \mathrm{S}\right. \\
\left.47^{\circ} 51^{\prime} \mathrm{W}\right)\end{array}$ & Oyster shell & After max & A 149 & $3090 \pm 110$ \\
\hline \multicolumn{7}{|c|}{ Geologic formations of Sao Paulo coast } \\
\hline-3438 & Comprida I. & $\begin{array}{l}\left(25^{\circ} 01^{\prime} \mathrm{S}\right. \\
\left.47^{\circ} 55^{\prime} \mathrm{W}\right)\end{array}$ & Wood & $0 \pm 0.3 \mathrm{~m}$ & A 23 & $680 \pm 90$ \\
\hline-3430 & Iguape I. & $\begin{array}{l}\left(24^{\circ} 51^{\prime} \mathrm{E}\right. \\
\left.47^{\circ} 28^{\prime} \mathrm{W}\right)\end{array}$ & Plant debris & $0.6 \pm 0.3 \mathrm{~m}$ & A 37 & $3370 \pm 100$ \\
\hline-3439 & Ararapiza channel & $\begin{array}{l}\left(25^{\circ} 09^{\prime} \mathrm{S}\right. \\
\left.48^{\circ} 02^{\prime} \mathrm{W}\right)\end{array}$ & Wood & $1.5 \pm 0.3 \mathrm{~m}$ & A 28 & $4400 \pm 110$ \\
\hline-3644 & Sao Paulo & $\begin{array}{l}\left(25^{\circ} 00^{\prime} \mathrm{S}\right. \\
\left.47^{\circ} 54^{\prime} \mathrm{W}\right)\end{array}$ & Oyster shell & $1.4 \pm 0.4 \mathrm{~m}$ & A 89 & $5410 \pm 120$ \\
\hline-3848 & Pernambuco, Santo & $\begin{array}{l}\left(23^{\circ} 58^{\prime} \mathrm{S}\right. \\
\left.\quad 46^{\circ} 11^{\prime} \mathrm{W}\right)\end{array}$ & Vermets & $+1.4 \pm 0.4 \mathrm{~m}$ & A 249 & $790 \pm 90$ \\
\hline-3847 & Bertigas Santos & $\begin{array}{l}\left(23^{\circ} 57^{\prime} \mathrm{S}\right. \\
\left.\quad 46^{\circ} 26^{\prime} \mathrm{W}\right)\end{array}$ & Wood & $\mathrm{Ca} 0 \mathrm{~m}$ & A 239 & $4100 \pm 100$ \\
\hline-3850 & Bertigas Santos & $\begin{array}{l}\left(23^{\circ} 50^{\prime} \mathrm{S}\right. \\
\left.46^{\circ} 08^{\prime} \mathrm{W}\right)\end{array}$ & Wood & $+0.8 \pm 0.4 \mathrm{~m}$ & A 256 & $6020 \pm 130$ \\
\hline-3846 & Bertigas Santos & $\begin{array}{l}\left(23^{\circ} 57^{\prime} \mathrm{S}\right. \\
\left.46^{\circ} 26^{\prime} \mathrm{W}\right)\end{array}$ & Wood & $+0.8 \pm 0.3 \mathrm{~m}$ & A 238 & $6280 \pm 130$ \\
\hline-3845 & Santos & $\begin{array}{l}\left(24^{\circ} 00^{\prime} \mathrm{S}\right. \\
\left.46^{\circ} 23^{\prime} \mathrm{W}\right)\end{array}$ & Wood & Below $\pm 1.5 \mathrm{~m}$ & A 234 & $6250 \pm 130$ \\
\hline-3844 & Santos & $\begin{array}{l}\left(23^{\circ} 57^{\prime} \mathrm{S}\right. \\
\left.46^{\circ} 24^{\prime} \mathrm{W}\right)\end{array}$ & Wood & $+3.5 \pm 0.5 \mathrm{~m}$ & A 225 & $\geq 35,000$ \\
\hline-3431 & Caraguatatuba Plain & $\begin{array}{l}\left(23^{\circ} 39^{\prime} \mathrm{S}\right. \\
\left.45^{\circ} 29^{\prime} \mathrm{W}\right)\end{array}$ & $\begin{array}{l}\text { Organic } \\
\text { matter }\end{array}$ & $+1.5 \pm 0.75 \mathrm{~m}$ & SP 02 & $3320 \pm 100$ \\
\hline-3433 & Caraguatatuba Plain & $\begin{array}{l}\left(23^{\circ} 39^{\prime} \mathrm{S}\right. \\
\left.45^{\circ} 29^{\prime} \mathrm{W}\right)\end{array}$ & Wood & $-12.5 \pm 1 \mathrm{~m}$ & SP-5 & $7950 \pm 220$ \\
\hline-3434 & Caraguatatuba bay & $\begin{array}{l}\left(23^{\circ} 39^{\prime} \mathrm{S}\right. \\
\left.45^{\circ} 29^{\prime} \mathrm{W}\right)\end{array}$ & $\begin{array}{l}\text { Organic } \\
\text { matter }\end{array}$ & $-16.2 \pm 1.9 \mathrm{~m}$ & SP0 05 & $8030 \pm 150$ \\
\hline-3647 & Ilha Grande bay & $\begin{array}{l}\left(23^{\circ} 00^{\prime} \mathrm{S}\right. \\
\left.45^{\circ} 00^{\prime} \mathrm{W}\right)\end{array}$ & Shell in situ & $+1.5 \pm 0.5 \mathrm{~m}$ & A 178 & $2390 \pm 100$ \\
\hline
\end{tabular}

Gif-3697. Maroni estuary, BSP 4

$4340 \pm 120$

110 to $130 \mathrm{~cm}$ under present soil.

Gif-3696. Maroni estuary, BSP 55

$4500 \pm 130$

80 to $200 \mathrm{~cm}$ under present soil.

General Comment: dates rapid marine transgression which reached ca $0.80 \mathrm{~m}$ above present msl, $4200 \mathrm{BP}$. 


\section{Mer de Champlain series, Quebec, Canada}

Shells from uplift levels in Mer de Champlain. Coll and subm 19751976 by J M Dubois, Univ Sherbrooke. Alts are related to present msl.

Gif-3769. Mer de Champlain, Rochemonteix

$7670 \pm 140$

$\left(50^{\circ} 17^{\prime} \mathrm{N}, 65^{\circ} 40^{\prime} \mathrm{W}\right)$, alt $+42.5 \mathrm{~m}$

Gif-3768. Mer de Champlain, Pointe Saint-Charle $\quad \mathbf{8 8 9 0} \pm 160$

$\left(50^{\circ} 17^{\prime} \mathrm{N}, 65^{\circ} 50^{\prime} \mathrm{W}\right)$, alt $+22.7 \mathrm{~m}$.

Gif-3770. Mer de Champlain,

Estuary de la Chaloupe R, $1 \quad 10,230 \pm 180$

$\left(50^{\circ} 11^{\prime} \mathrm{N}, 65^{\circ} 01^{\prime} \mathrm{W}\right)$, alt $\mathrm{ca}+3 \mathrm{~m}$.

\section{Gif-4017. Mer de Champlain,}

Estuary de la Chaloupe R, $2 \quad 9630 \pm 200$

$\left(50^{\circ} 17^{\prime} \mathrm{N}, 65^{\circ} 07^{\prime} \mathrm{W}\right)$, alt $+3 \mathrm{~m}$. Comment: confirms age of level $+3 \mathrm{~m}$ dated by Gif-3770, and dates beginning of estuarian sedimentation phase.

General Comment: set of very coherent dates for rapid uplift of coast after ice-cap retreat in this region.

\section{Taiaro atoll series, Tuamotu, Polynesia}

Coral from lagoon of Taiaro, one of smallest atolls from Tuamotu Is., diam $5 \mathrm{~km}\left(15^{\circ} 44^{\prime} \mathrm{S}, 144^{\circ} 10^{\prime} \mathrm{W}\right)$. Coll and subm 1973 by J P Chevallier, Inst Paleont, Mus Hist Nat, Paris. Lagoon is presently closed and water level at $0.60 \mathrm{~m}$ under high sea level. Samples from ancient "hoa" which was passage of oceanic water.

Gif-2920. Taiaro, TAI 8

$1120 \pm 80$

From $1 \mathrm{~m}$ above present lagoon water level.

Gif-2921. Taiaro, TAI 28 a

$1090 \pm 80$

From reef, ca $0.70 \mathrm{~m}$ above present lagoon water level.

General Comment: dates closing of lagoon, probably due to uplift of atoll at AD 660 to 1160 .

\section{Volcanism}

Gif-2960. Tartaret, Chambon Lake, France

$2970 \pm 110$

Paleosol underlying basalt flow, Tartaret $\left(45^{\circ} 33^{\prime} \mathrm{N}, 2^{\circ} 54^{\prime} \mathrm{E}\right)$. Coll and subm 1974 by R Brousse, Fac Sci, Orsay.

Gif-3425. Las Lajas, Tenerife I, Canary Is.

$8760 \pm 160$

Charcoal from humic level at base of andosol formed on volcanic ash overlying tropical brown soil $\left(28^{\circ} 15^{\prime} \mathrm{N}, 16^{\circ} 35^{\prime} \mathrm{W}\right)$. Coll and subm 1974 by $\mathrm{P}$ Quantin, ORSTOM, Bondy. Comment: gives min age for ash. 
Gif-3085. Mangoum-Foumbo, Cameroun

$1290 \pm 80$

Charcoal in humic level under basaltic scorias, $140 \mathrm{~cm}$ thick Babileke Plateau $\left(5^{\circ} 39^{\prime} \mathrm{N}, 10^{\circ} 45^{\prime} \mathrm{E}\right)$, alt $1500 \mathrm{~m}$. Coll by $\mathrm{P}$ Muller and subm 1974 by F Paris and G Sieffermann. Comment: dates one of last volcanic events in this region.

Gif-3487. Kasbeghi, Caucasus

$6290 \pm 90$

Wood fragments under lava flow in Terek valley. Coll and subm 1971 by G Montjuvent. Comment: dates last lava flow of Kasbek volcano.

Mexico

\section{S E Mexico series}

Samples dated to establish chronology of volcanism of SE Mexico. Geol study by C Robin (1981).

\section{Pico de Orizaba series}

Charcoal from Pico de Orizaba Volcano, coll 1979-1980 by P Vincent, C Robin, and J Cantagrel, Inst Geog, Clermont-Ferrand, France.

Gif-4952. Coscomatepec valley, PU 110 $7020 \pm 130$

Carbonized tree trunk in nuée ardente deposit of Saint-Vincent type $\left(18^{\circ} 58^{\prime} \mathrm{N}, 97^{\circ} 05^{\prime} \mathrm{W}\right)$. Coll and subm by $\mathrm{P}$ Vincent.

Gif-4953. Hidalgo, PU 107 thick.

Carbonized wood in gray pumice underlying volcanic debris $10 \mathrm{~m}$

Gif-5188. Loma Grande, PU 127, Mexico

$6200 \pm 120$

Charcoal in lowest pyroclastic flow deposit of Saint-Vincent type $\left(18^{\circ}\right.$ $\left.55^{\prime} \mathrm{N}, 97^{\circ} 14^{\prime} \mathrm{W}\right)$. Alt $2630 \mathrm{~m}$.

Gif-5189. Texmalaca Orizaba valley, PU 132

$3400 \pm 110$

Charcoal in upper pyroclastic flow deposit of Saint-Vincent type $\left(18^{\circ}\right.$ $\left.58^{\prime} \mathrm{N}, 97^{\circ} 06^{\prime} \mathrm{W}\right)$. Alt $1630 \mathrm{~m}$.

Gif-5190. El Paso, Hidalgo Coscomatepec, PU $139 \quad 4060 \pm 120$

Charcoal in ocher pumice deposit $\left(19^{\circ} 12^{\prime} \mathrm{N}, 97^{\circ} 12^{\prime} \mathrm{W}\right)$. Alt $3580 \mathrm{~m}$.

Gif-5191. Hidalgo Coscomatepec road, PU $141 \quad 10,600 \pm 190$

Charcoal in nuée ardente deposit of Saint-Vincent type $\left(19^{\circ} 12^{\prime} \mathrm{N}, 77^{\circ}\right.$ $\left.12^{\prime} \mathrm{W}\right)$. Alt $3450 \mathrm{~m}$.

Gif-5192. Quarries of Chocaman, PU 143

$12,900 \pm 150$

Charcoal in pyroclastic flow deposit $\left(19^{\circ} 01^{\prime} \mathrm{N}, 96^{\circ} 56^{\prime} \mathrm{W}\right)$.

Gif-5193. Teteltzingo, PU 146

$9400 \pm 170$ 


\section{Popocatepetl series}

Samples from Popocatepetl volcano, coll in nuée ardente deposit of Saint-Vincent type and subm 1982 by J Cantagrel, Inst Geog, ClermontFerrand.

Gif-5594. Paso de Cortes sec, PO 60

Carbonized wood at base of nuée ardente deposit, $\left(19^{\circ} 05^{\prime} \mathrm{N}, 98^{\circ} 36^{\prime}\right.$ W). Alt $3250 \mathrm{~m}$.

Gif-5595. Quarry of Bavanco Seca, Canado, PO $61 \quad 4320 \pm 70$

Carbonized wood in lower nuée ardente deposit with dacitic pumice, Quarry of Bavanco Seca Huiloclatengo, E side of volcano $\left(19^{\circ} 05^{\prime} \mathrm{N}, 98^{\circ}\right.$ $\left.36^{\prime} \mathrm{W}\right)$. Alt $3600 \mathrm{~m}$.

Gif-5596. Quarry of Bavanco Seca, Canado, PU 74 $9640 \pm 440$

Carbonized wood in lowest tephra deposit, alternately gray and ocher ash; in same quarry as Gif-5595.

Gif-5597. San Pedro Benito Juarez, PO 65

$1220 \pm 60$

Carbonized wood at base of nuée ardente deposit of Saint-Vincent type, $\left(18^{\circ} 57^{\prime} \mathrm{N}, 98^{\circ} 33^{\prime} \mathrm{W}\right)$. Alt $2060 \mathrm{~m}$.

Gif-5598. Tlaltzopia, PO 72

$880 \pm 80$

Carbonized wood in lowest nuée ardente deposit of Saint-Vincent type with large blocks, W Santiago Xalizintla $\left(19^{\circ} 04^{\prime} \mathrm{N}, 98^{\circ} 32^{\prime} \mathrm{W}\right)$. Alt $3850 \mathrm{~m}$. General Comment: dates indicate very recent pyroclastic activity previously unknown.

Gif-4954. Quarry of Rio Frio, RF 1

Carbonized wood at base of pyroclastic flow deposit of pumice and ash Coll by P Vincent.

\section{Nevado de Toluca series}

Samples from Nevado de Toluca volcano. Coll and subm 1979-1980 by P Vincent and C Robin.

Gif-4955. Barranca de Jahal, NE 12

Paleosol.

Gif-5187. Rio Grande Valley, Barranca de Tlamisco, NE 30

Charcoal in lower Pelean nuée ardente deposit $\left(19^{\circ} 02^{\prime} \mathrm{N}, 99^{\circ} 39^{\prime}\right)$. 


\section{Lacustrine Variations in Africa}

Mauritania

\section{Tiriersoum series}

Samples of calcareous lacustrine sediment from ancient lake in Tiriersoum region $\left(21^{\circ} 15^{\prime} \mathrm{N}, 16^{\circ} 22^{\prime} \mathrm{W}\right)$. Coll and subm 1971 by $\mathrm{G}$ Delibrias.

Gif-2501. Tiriersoum, $0-5 \mathrm{~cm}$ $3000 \pm 100$

Gif-2502. Tiriersoum, $35-40 \mathrm{~cm}$ $4290 \pm 110$

Gif-2503. Tiriersoum, $75-85 \mathrm{~cm}$ $4560 \pm 110$

Gif-2504. Tiriersoum, $125-130 \mathrm{~cm}$ $7690 \pm 140$

General Comment: dates Holocene lacustrine episode in W Africa. After diatoms study (F Gasse) deepest lake occurred 4290 BP. Surface sediment, very poor in diatoms, corresponds to time when lake dried up.

\section{Azefal series}

Lacustrine sediment, depth $50 \mathrm{~cm}$, at Graref Agourega $\left(19^{\circ} 50^{\prime} \mathrm{N}, 16^{\circ}\right.$ $\left.10^{\prime} \mathrm{W}\right)$. Coll and subm by L Hebrard, IFAN, Dakar.

\section{Gif-2500. Azefal, 1 Carbonate fraction}

$$
\begin{array}{r}
4550 \pm 110 \\
\delta^{13} C=-4.3 \% o \\
\mathbf{4 4 9 0} \pm \mathbf{1 1 0} \\
\delta^{13} C=-17.5 \% 0
\end{array}
$$

Gif-2500 bis. Azefal, 2 Organic fraction

General Comment: both fractions give same age within statistical margin of dates.

\section{Gif-2506. Goud Anagoum}

$4560 \pm 110$

Gasteropods from lacustrine deposit between sand dunes of Akchar and Agueitir $\left(19^{\circ} 19^{\prime} \mathrm{N}, 16^{\circ} 08^{\prime} \mathrm{W}\right)$. Coll and subm 1972 by L Hebrard. Comment: corresponds to Holocene lacustrine episode.

Gif-2549. MAU 72-8

$2250 \pm 100$

Gasteropod shells from dried lagoon behind range of dunes $\left(17^{\circ} 28^{\prime} \mathrm{N}\right.$, $15^{\circ} 58^{\prime} \mathrm{W}$ ). Coll and subm 1972 by J P Carbonnel. Comment: confirms dates already pub for end of last moist period in W Africa (Delibrias, Guillier \& Labeyrie, 1982).

\section{Gif-2505. Tintane}

$$
\begin{array}{r}
6070 \pm 130 \\
\delta^{13} C=-5.2^{0} \%
\end{array}
$$

Plant roots calcified in sand from Neolithic site, Tintane $\left(20^{\circ} 55^{\prime} \mathrm{N}, 16^{\circ}\right.$ $40^{\prime} \mathrm{W}$ ). Coll and subm 1971 by J P Carbonnel. Comment: confirms existence of moist period $6000 \mathrm{yr}$ ago in Mauritania. Data agree very well with Holocene lacustrine extension of Aouker Lake (below). 


\section{Aouker Lake series}

Samples dated to precise extension of Aouker Lake (Hugot, 1977) in late Quaternary time. Coll around Tichitt $\left(18^{\circ} 28^{\prime} \mathrm{N}, 9^{\circ} 28^{\prime} \mathrm{W}\right)$ and near Akhreijit ( $\left.18^{\circ} 20^{\prime} \mathrm{N}, 9^{\circ} 10^{\prime} \mathrm{W}\right)$ and subm 1971-1972-1973 by H J Hugot, Inst Paleontol Humaine, Paris.

Gif-2878. Tichitt, TIC 18/72

$8180 \pm 170$

Organic level in sand overlying diatomite.

$\begin{array}{lr}\text { Gif-2879. Tichitt, TIC 71/10-5 } & 8570 \pm 170\end{array}$

Calcareous diatomite level.

Gif-2883. Tichitt, TICH 235

$6990 \pm 150$

Calcareous diatomite deposit with faluns, behind sand-hill.

Gif-2885. Tichitt, TICH 247/4

$7120 \pm 150$

Calcareous diatomite lying on paleozoic substratum.

Gif-2876. Tichitt, TIC 2/72

$4570 \pm 130$

Calcareous diatomite from base of lake.

Gif-2887. Tichitt, T, HAF 71/1

$4670 \pm 130$

Calcareous diatomite with faluns.

Gif-3405. Akhreijit, Monodville, 24

$5030 \pm 110$

Hippopotamus bones, under lacustrine deposit.

Gif-3406. Akhreijit, Monodville, sup B

$730 \pm 90$

Bones in ashy level.

Gif-4110. Tichitt, TIC 70

$2610 \pm 110$

Fish bones, from hearth, in Neolithic site on border of lake. Comment: dates limit for drying of lake.

Gif-4111. Tichitt, TIC 75

$1930 \pm 110$

Human bones from necropole of Tichitt village. Comment: dates last settlement in village.

Gif-4112. Tichitt, TIC 75

$2290 \pm 110$

Hippopotamus bones from Neolithic site on lacustrine deposit. Comment: dates end of pachyderma fauna.

General Comment: date beginning and extension of Aoukert Lake, at Holocene time and its drying ca $2500 \mathrm{BP}$. 
Ethiopia

\section{Ziway-Shala Lake basin, S Ethiopia}

\section{Bulbula R, B 20 series}

Samples from Sec $10 \mathrm{C}$ in Bulbula lacustrine fm, between Ziway and Abiyata Lakes $\left(7^{\circ} 48^{\prime} \mathrm{N}, 38^{\circ} 41^{\prime} \mathrm{E}\right)$. Coll and subm 1975 by A Street-Perrot, Univ Oxford, England.

Gif-3984. Sec $10 \mathrm{C}$, no. 1 $8520 \pm 200$

Organic matter from paleosol underlying calcareous silt deposit.

Gif-3985. Sec $10 \mathrm{C}$, no. 2

$12,200 \pm 200$

Carbonate fraction from diatomaceous marl deposit overlying paleosol. Comment: corresponds to high stand of lake, called Bulbula III.

Gif-3986. Sec 10 C, no. 3

$10,450 \pm 180$

Organic matter from soil underlying Gif-3985. Comment: from same level, charcoal was dated at $11,870 \pm 300$ by G Gillespie; this discrepancy is unexplained.

Gif-3987. Sec 10 C, no. 4

$22,050 \pm 650$ sand.

Carbonate fraction from calcareous silt overlying pebbly pumice

Gif-3988. Sec 10 C, no. 5

$\mathbf{2 4 , 0 0 0} \pm \mathbf{7 5 0}$

Carbonate fraction from base level from same deposit as Gif-3987. Comment: date corresponds to high stand of lake, called Bulbula II.

Gif-4011. Shala Lake, Sec 39B

$2510 \pm 100$

Charcoal from top of colluvial loams deposit around Shala Lake. Coll and subm 1977 by A Street-Perrott. Comment: corresponds to arid phase of lake.

\section{Bulbula Wadi series, Sec GO series}

Shells from Bulbula lacustrine deposit, between Ziway and Abiyata Lakes $\left(8^{\circ} \mathrm{N}, 39^{\circ} \mathrm{E}\right)$. Coll by J Gèze and subm 1973 by $\mathrm{P}$ Rognon.

Gif-2817. Bulbula, G O 1

$4960 \pm 140$

Shelly level overlying diatomite deposit.

Gif-2818. Bulbula, G O 4

$9360 \pm 210$

Shelly level under diatomite level.

Gif-4010. Bulbula R, Sec B 26

$28,200 \pm 1000$

Shells (Corbicula sp) from section very close to exit from Ziway Lake $\left(7^{\circ}\right.$ $47^{\prime} \mathrm{N}, 38^{\circ} 44^{\prime} \mathrm{E}$ ), under fine sand with pumice pebbles, $1.5 \mathrm{~m}$ thick. Coll and subm 1977 by A Street-Perrott. Comment: corresponds to lowstand of lake. 


\section{Bulbula R, Sec B 3 series}

Shelly horizon underlying silty sand deposit with surface soil at top, overlying Pleistocene diatomite sequence $\left(7^{\circ} 47^{\prime} \mathrm{N}, 38^{\circ} 45^{\prime} \mathrm{E}\right)$. Different species of shells were separated and dated. Coll and subm 1977 by A StreetPerrott.

Gif-4012. Sec B 3, A

Shells (Bellamya unicolor).

Gif-4013. Sec B 3, B

Shells (Melanoïdes tuberculata).

Gif-4014. Sec B 3, C

Shells (Bulinus truncatus). Comment: dates highstand of Holocene lake as expected; there is no difference between ages of different species.

General Comment: for Ziway-Shala Lake Basin, chronological study of lacustrine sequences in this basin establishes precise dates and confirms magnitude and abruptness of lake-level fluctuations in $\mathrm{N}$ intertropical Africa during Late Quaternary time (Gillespie, Street-Perrot \& Switsur, 1983).

\section{Ghana}

\section{Bosumtwi Lake series}

Bosumtwi Lake $\left(6^{\circ} 30^{\prime} \mathrm{N}, 1^{\circ} 25^{\prime} \mathrm{W}\right)$ lies within steep-sided crater that was produced by meteoric impact. Lake level is, thus, largely dependent upon rainfall over the crater. Consequently, study of changing lake levels is a particularly valuable paleoclimatic tool to reconstruct atmospheric conditions over W Africa during Late Quaternary (Talbot, 1980). Present max water depth is $80 \mathrm{~m}$. Overflow channel is ca $45 \mathrm{~m}$ above present lake level (apll). Samples (Table 5) coll and subm 1976-1979 by M R Talbot, Univ Bergen, Norway.

Shell and algal carbonate dates were corrected for isotopic fractionation, according to $\delta^{13} \mathrm{C}$ values measured, from $0.6 \%$ to $2.7 \% 0$.

\section{Gif-4605. Bosumtwi BO 78:2}

$$
\begin{array}{r}
\Delta^{14} \mathbf{C}=+272 \pm 5 \% \\
\delta^{13} C=-13.8 \% 0
\end{array}
$$

Living shells (Bulinus sp) coll at Banso in Abiriw stream, in 1978. Comment: ${ }^{14} \mathrm{C}$ content of these mollusk shells is in approx equilibrium with atmospheric ${ }^{14} \mathrm{C}$ and is evidence of very low "hard effect" and reliability of shell and carbonate dating in vicinity of lake.

General Comment: during Pleistocene, Lake Bosumtwi suffered major regression and probably remained low for some time. Just after 13,000 BP, lake rose again and thereafter crater was generally characterized by lake significantly higher than at present. These periods of high water level were interrupted by short, but intense regression ca 10,500-8000, 4500-4000 and just after $1000 \mathrm{BP}$ (Talbot \& Delibrias, 1977, 1980; Talbot et al, 1984). 
TABLE 5

Bosumtwi Lake, Ghana

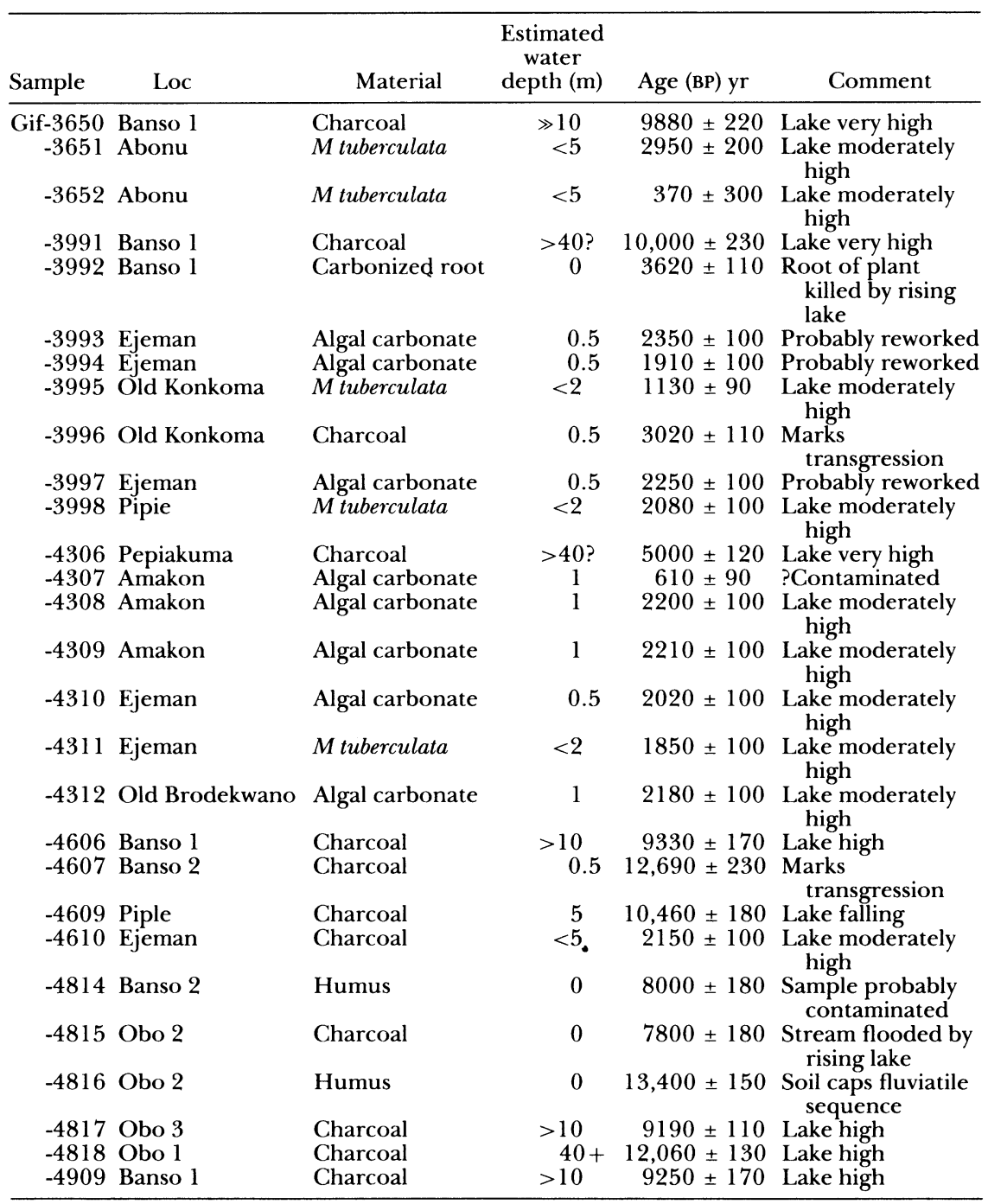

Niger

Gif-3892. Dogomboulo, Fachi, Niger, C

$12,080 \pm 220$

Organic matter from base level of Holocene lacustrine sequence, $2 \mathrm{~m}$ depth $\left(18^{\circ} 06^{\prime} \mathrm{N}, 11^{\circ} 34^{\prime} \mathrm{E}\right)$. Coll and subm 1976 by J Maley. 
Chad

\section{Mouskorbé series, E Tibesti}

Samples from lacustrine depression, Mouskorbé Mts $\left(21^{\circ} 11^{\prime} \mathrm{N}, 18^{\circ}\right.$ $\left.25^{\prime} \mathrm{E}\right)$. Alt $+2700 \mathrm{~m}$. Coll 1968 by B Messerli and subm 1974 by J Maley, ORSTOM, Montpellier.

Gif-3228. Mouskorbé, 3

$6600 \pm 140$

Plant remains in marshy paleosol with diatoms.

Gif-4182. Mouskorbé, $X$

$6700 \pm 140$

Calcareous encrusting deposited on border of depression.

General Comment: dates beginning of arid phase, at that alt, in E Tibesti.

\section{Kouka series}

Samples from test pit in lacustrine sediments, Kouka $\left(13^{\circ} 06^{\prime} \mathrm{N}, 15^{\circ} 38^{\prime}\right.$ E), $20 \mathrm{~km}$ from E Chad Lake. Alt $+283 \mathrm{~m}$. Subm 1975 by J Maley.

Gif-3682. Kouka, 2

$110 \pm 90$

Shells (Unio sp) in silty level under $0.65 \mathrm{~m}$ black clay level.

Gif-3681. Kouka, 1

$360 \pm 90$

Shells (Unio sp) in silty level, 1.20 to $1.45 \mathrm{~m}$ depth.

Gif-4232. Kouka, 3

Shells from base level from silty level.

Gif-3548. Kouka, 3

$9690 \pm 210$

Organic sediment, $5.90 \mathrm{~m}$ depth, overlying clayey sand and overlain by lacustrine silty clayey deposit. Comment: dates beginning of important transgressive period.

General Comment: Gif-3682, -3681, and -4232 date recent and probably very short lower level of Chad. These young ages, mainly Gif-4232, $\leq 90 \mathrm{yr}$, show no "hard effect" and indicate validity of ${ }^{14} \mathrm{C}$ dates for lacustrine shells in Chad region.

\section{Gif-3549. Ngouri}

$260 \pm 120$

Shells (Limicolaria sp) $0.50 \mathrm{~m}$ depth in gray silt in ancient course of Bahr el Ghazal $\left(13^{\circ} 39^{\prime} \mathrm{N}, 15^{\circ} 23^{\prime} \mathrm{E}\right)$. Coll and subm 1975 by J Maley. Comment: very recent age of shells show very little "hard effect" in Chad region.

\section{Gif-3610. Tjeri, 1588}

$12,500 \pm 220$

Organic matter from base level of lacustrine sequence, $12.20 \mathrm{~m}$ depth $\left(13^{\circ} 45^{\prime} \mathrm{N}, 16^{\circ} 30^{\prime} \mathrm{E}\right)$. Coll by M Servant and subm 1975 by J Maley.

\section{Gif-4125. Mandi}

$10,000 \pm 170$

Organic matter from regressive sediment level, $4 \mathrm{~m}$ depth in core $\mathrm{H} 9$ $\left(13^{\circ} 27^{\prime} \mathrm{N}, 14^{\circ} 40^{\prime} \mathrm{E}\right)$. Coll by B Dupont and subm 1977 by J Maley. 


\section{Gif-4233. Dilindri}

Shells coll and subm 1977 by J Maley.

General Comment: for Chad samples: dated lacustrine deposits belong to two transgressive lacustrine periods $12,500-9600 \mathrm{BP}$ and ca $6000 \mathrm{BP}$. Present Chad Lake is remains of Paleochad which extended to area similar to that of Caspian Sea, between 6000-5000 BP.

\section{Central Afar}

Central Afar, now desert, is in Ethiopia and Djibouti Republic, at $\mathrm{E}$ end of Sahelian African zone. Its numerous tectonic depressions have been filled up by large lakes during Upper Quaternary humid periods. Lake level fluctuations are deduced from parallel analysis of numerous geol secs, and of one core, on border of Abhe Lake. Chronology of main stages of lacustrine evolution was established. Paleo-environments were also reconstructed from sedimentary facies and diatom flora (Gasse, 1975). Samples from Afar were coll and subm 1971-72-73-74 by F Gasse and P Rognon, CNRS, Paris, and samples from Ethiopia were coll and subm 1975 by A Street-Perrott.

Gif-2715. Assaita plain, A 100

$8000 \pm 170$

Shells (mainly Unio $\mathrm{sp}$ ) in conglomerate overlying silty diatomite $\left(11^{\circ}\right.$ $\left.39^{\prime} \mathrm{N}, 41^{\circ} 29^{\prime} \mathrm{E}\right)$.

\section{Gif-2571. Assaita plain, M 161}

$13,130 \pm 170$

Calcareous level with plant remains, at top of diatomite A 80 bis, overlain by silty diatomite without shells $\left(11^{\circ} 38^{\prime} \mathrm{N}, 41^{\circ} 53^{\prime} \mathrm{E}\right)$.

Gif-2716. Assaita plain, A 80 bis

$23,600 \pm 740$

Shells (mainly Melania sp) in diatomite ( $\left.11^{\circ} 39^{\prime} \mathrm{N}, 41^{\circ} 29^{\prime} \mathrm{E}\right)$.

Gif-2572. Assaita plain, NW Barawle volcano

$8330 \pm 180$

Shells (Unio and Melania sp) from beach deposit left at time of extension of lakes of Awash low valley ( $\left.11^{\circ} 30^{\prime} \mathrm{N}, 41^{\circ} 20^{\prime} \mathrm{E}\right)$.

\section{Erole II series, $\mathbf{S}$ Magenta}

Samples from Erole II sec ( $\left.11^{\circ} 19^{\prime} \mathrm{N}, 40^{\circ} 20^{\prime} \mathrm{E}\right)$, in Awash valley.

\section{Gif-2727. Erole II, A 59}

$$
31,000+\frac{2200}{-1700}
$$

Shells (Melania sp) from shelly level at base of pure diatomite deposit, $5 \mathrm{~m}$ thick, underlying Gif-2718.

Gif-2894. Erole II, A 45

$$
\begin{array}{r}
30,600 \\
+1700
\end{array}
$$

Calcareous diatomite overlying Gif-2727. 
Gif-2718. Erole II, A 60

$16,800 \pm 310$

Carbonate from indurated calcareous slab, interstratified in diatomite Gif-2894. Comment: date strongly disagrees with stratigraphy and is unexplained.

\section{Asal Lake series}

Samples from ancient sediment of Asal Lake $\left(11^{\circ} 45^{\prime} \mathrm{N}, 42^{\circ} 25^{\prime} \mathrm{E}\right)$.

Gif-2719. W Asal L, A 167

$5300 \pm 130$ Lake.

Lamellibranch shells from base level of gypseous sediments of Asal

Gif-2720. W Asal L, A 166

$8540 \pm 180$

Shells (Melania sp) from silty level separated from A 167 by calcareous deposit, $1.5 \mathrm{~m}$ thick.

Gif-3063. N Asal L, B 183

$\geq \mathbf{4 0 , 0 0 0}$

Shells from base of diatomite.

\section{E Magenta Plateau series}

Samples from different secs of E border Magenta plateau $\left(11^{\circ} 00^{\prime} \mathrm{N}\right.$, $\left.41^{\circ} 20^{\prime} \mathrm{E}\right)$.

Gif-2810. E Magenta A 25

$8650 \pm 160$

Shells (Unio, Melania sp).

Gif-2811. E Magenta A 26

$8450 \pm 190$

Shells (Melania sp) from silty level, superimposed on A 25.

Gif-2816. E Magenta, A 22

$8770 \pm 190$

Shells.

Gif-2812. E Magenta, A 30

$7460 \pm 180$

Shells from silty level.

Gif-2814. E Magenta, A 32

$6020 \pm 160$

Shells overlying silty level.

General Comment: date extension of lower Holocene lacustrine phase, between 8000 and 9000 BP.

Gif-2813. Dubti, A 6

$8530 \pm 190$

Shells in calcareous encrusting $\left(11^{\circ} 44^{\prime} \mathrm{N}, 41^{\circ} 04^{\prime} \mathrm{E}\right)$.

Gif-2815. Dit-Bahari, A 11

$8450 \pm 190$

Shells (Unio, Melania sp) $\left(11^{\circ} 35^{\prime} \mathrm{N}, 41^{\circ} 10^{\prime} \mathrm{E}\right)$. 
As Ela sec series, Gobaad plain

Shells from calcareous diatomite deposit, $1 \mathrm{~m}$ thick $\left(11^{\circ} 00^{\prime} \mathrm{N}, 42^{\circ} 07^{\prime}\right.$ E).

Gif-2854. As Ela, A 643

$4120 \pm 110$

At top level of diatomite.

Gif-2855. As Ela, A 645

$7610 \pm 140$

At base level of diatomite.

General Comment: date transgression of Abhe Lake at Middle Holocene.

Gif-3402. Modahtou, B 87

$7150 \pm 100$

Shells in clayey level, near Abhe Lake ( $\left.10^{\circ} 59^{\prime} \mathrm{N}, 41^{\circ} 49^{\prime} \mathrm{E}\right)$.

Gif-3093. As Ela, B 108

$26,000 \pm 700$

Shells in calcareous deposit $\left(10^{\circ} 59^{\prime} \mathrm{N}, 42^{\circ} 08^{\prime} \mathrm{E}\right)$.

\section{Barogali Wadi series, Gobaad plain}

Shells from superimposed levels corresponding to 2 different periods of lacustrine extension $\left(11^{\circ} 06^{\prime} \mathrm{N}, 42^{\circ} 04^{\prime} \mathrm{E}\right)$.

Gif-3064. Barogali W, B 428

$7450 \pm 100$

Melanoïdes sp, from shelly level. Comment: dates max extension of Abhe Lake at Middle Holocene.

Gif-3065. Barogali W, B 427

$\mathbf{2 0 , 8 0 0} \pm \mathbf{4 0 0}$

Calcareous diatomite deposit. Comment: dates beginning of regression of Abhe Lake at Lower Pleistocene.

Gif-3066. Gramari Lake, B 132

$24,800 \pm 650$

Shells in brown silt, $\mathrm{S}$ E present residual Gramari Lake in low valley of Awash $\left(11^{\circ} 29^{\prime} \mathrm{N}, 41^{\circ} 42^{\prime} \mathrm{E}\right)$. Comment: dates max of late transgression of Abhe Lake at Lower Pleistocene.

Gif-2859. Sankal-Godaad, A 648

E).

Shells (Unio sp) from shelly beach, in Godaad plain $\left(11^{\circ} 00^{\prime} \mathrm{N}, 42^{\circ} 30^{\prime}\right.$

\section{Hanle series}

Shells from sec in shelly lacustrine deposit $\left(11^{\circ} 21^{\prime} \mathrm{N}, 42^{\circ} 10^{\prime} \mathrm{E}\right)$.

Gif-2857. Hanle, A 527 b

$5250 \pm 120$ sode.

From top of calcareous level. Comment: corresponds to Holocene epi-

Gif-3061. Hanle, B 277

$\geq 35,000$

From basal level. Comment: corresponds to 2nd lacustrine episode of Upper Pleistocene. 
Unda-Dobi series

Samples from Unda-Gobi graben $\left(11^{\circ} 42^{\prime} \mathrm{N}, 41^{\circ} 51^{\prime} \mathrm{E}\right)$.

Gif-2858. Unda-Gobi, A 569

$6570 \pm 130$

Shells from top of calcareous diatomite deposit, $3 \mathrm{~m}$ thick. Comment: corresponds to lacustrine optimum.

Gif-3092. Unda-Gobi, B 293

$23,600 \pm 650$

Calcareous ashy diatomite. Comment: dates $\mathrm{N}$ extension of lakes during Upper Pleistocene.

Gif-3062. Sankal Wadi, B 36

Shells (Unio sp) in silt, from lacustrine beach on right side of Sankal W $\left(11^{\circ} 00^{\prime} \mathrm{N}, 42^{\circ} 10^{\prime} \mathrm{E}\right)$.

\section{Sediment core series, Abhe Lake, Djibouti Republic}

Samples from sediment core, $50 \mathrm{~m}$ depth, on border of present Abhe Lake ( $\left.11^{\circ} 08^{\prime} \mathrm{N}, 41^{\circ} 53^{\prime} \mathrm{E}\right)$. Subm 1973 by F Gasse. Present Abhe Lake, alt $+240 \mathrm{~m}$, is remains of very large lake which spread through low valley of Awash R, mainly at low Holocene (Table 6).

TABLE 6

Lake Abhe core

\begin{tabular}{|c|c|c|c|c|c|c|}
\hline Sample & $\begin{array}{l}\text { Depth } \\
(\mathrm{m})\end{array}$ & Sediment nature & Dated material & $\begin{array}{c}\mathrm{C} \\
\text { content } \\
(\%)\end{array}$ & $\begin{array}{l}\delta^{13} \mathrm{C} \\
(\% 0)\end{array}$ & Age (yr BP) \\
\hline Gif-3387 & $6.30-6.37$ & Calcareous clay & Carbonate & 4 & & $5830 \pm 90$ \\
\hline \multirow[t]{2}{*}{-3386} & $7.00-7.05$ & Calcareous clay & Carbonate & 8.5 & & $6660 \pm 100$ \\
\hline & & Calcareous clay & Organic matter & 3.5 & & $7000 \pm 140$ \\
\hline-3385 & $8.25-8.50$ & Calcareous clay & Organic matter & 2.4 & & $7250 \pm 140$ \\
\hline-3662 & $8.90-9.10$ & Calcareous clay & Organic matter & 1.5 & & $16,500 \pm 400$ \\
\hline-3095 & $9.84-10.04$ & Calcareous clay & Organic matter & 0.16 & & $16,100 \pm 800$ \\
\hline-3560 & $10.60-11.00$ & Calcareous clay & Organic matter & 0.13 & & $16,900 \pm 500$ \\
\hline-3091 & $13.70-13.82$ & Calcareous silt & Carbonate & 0.4 & & $17,100 \pm 400$ \\
\hline-3388 & $17.17-17.22$ & Calcareous clay & Carbonate & 1.4 & & $23,900 \pm 700$ \\
\hline-3084 & $18.98-19.28$ & $\begin{array}{l}\text { Clayed calcareous } \\
\text { diatomite }\end{array}$ & Carbonate & 5.8 & -1.5 & $25,100 \pm 700$ \\
\hline-3660 & $19.75-20.08$ & $\begin{array}{l}\text { Clayed calcareous } \\
\text { diatomite }\end{array}$ & Organic matter & 1.7 & -24.3 & $25,600 \pm 700$ \\
\hline-3659 & $20.80-21.08$ & $\begin{array}{l}\text { Clayed calcareous } \\
\text { diatomite }\end{array}$ & Organic matter & 2.6 & & $39,000 \pm 4000$ \\
\hline-3955 & $25.60-25.77$ & Calcareous clay & Carbonate & 1.9 & & $7830 \pm 140$ \\
\hline-3485 & $25.77-26.35$ & Calcareous clay & Organic matter & 0.7 & -2.5 & $9000 \pm 180$ \\
\hline-3364 & $26.85-27.19$ & Calcareous clay & Organic matter & 1.8 & -24.7 & $7830 \pm 140$ \\
\hline-3562 & $27.67-27.77$ & Calc & $\mathrm{Ca}$ & 3.6 & & $\geq 41,000$ \\
\hline-3563 & $29.53-29.63$ & Calcareous clay & Carbonate & 7 & -0.2 & $\geq 41,000$ \\
\hline
\end{tabular}

General Comment: dates help reconstruct successive phases of lacustrine evolution (Gasse, 1977). Large and persistent lakes extended in Afar and Main Rift from 40,000 and 20,000 yr with slight regression ca 30,000; 
highest levels in Pleistocene are dated ca 25,000-23,000. After arid phase, all lakes transgress ca $9000 \mathrm{BP}$ and reach high level between 9100-6000 BP. From 4000 BP onwards, climatic deterioration progressively incurred present situation. Diatomite studies indicate Late Pleistocene and Holocene environmental changes in subdesert $\mathrm{E}$ African zone. This study completes and verifies data on climatic evolution in Intertropical Africa, (Gasse \& Delibrias. 1976).

\section{Climatic samples}

\section{France}

Gif-3229. Grangues, Calvados, 907 $3640 \pm 100$

Silty peat from peat bog $\left(49^{\circ} 15^{\prime} \mathrm{N}, 0^{\circ} 03^{\prime} \mathrm{W}\right)$. Coll and subm 1974 by $\mathrm{E}$ Helluin, Centre Géomorphol, Caen. Comment: date does not confirm Atlantic period deduced from pollen analysis.

\section{Saint-André de Cubzac series, Dordogne}

Peaty clayey sediment from marsh in Dordogne Valley $\left(44^{\circ} 59^{\prime} \mathrm{N}, 0^{\circ}\right.$ $27^{\prime}$ W). Coll and subm 1973 by J F Vidalie, Lab Central Ponts et Chaussées, Paris.

Gif-3445. Saint-André de Cubzac, A 02

$5510 \pm 120$

Peaty level, depth $2.62 \mathrm{~m}$, overlying mud, $4 \mathrm{~m}$ thick.

Gif-3446. Saint-André de Cubzac, A 09

Peaty clayey level, depth $7 \mathrm{~m}$, underlying mud.

General Comment: both dates are within statistical limits of measurements; mud was deposited suddenly. Samples were dated for study of mechanical characteristics of compressible soils.

Gif-3486. La Rivière, Isère

$10,600 \pm 140$

Peaty gray clay at top of low terrace of glacial depression of Moirans $\left(45^{\circ} 20^{\prime} \mathrm{N}, 5^{\circ} 34^{\prime} \mathrm{E}\right)$. Coll and subm 1975 by $\mathrm{G}$ Montjuvent, Inst Dolomieu, Grenoble. Comment: dates retreat of glaciers in Isère Valley.

Gif-2561. Plain of Ariège R, Haute Garonne

$3410 \pm 110$

Wood from fossil tree in gravel pit, low alluvial plain of Ariège $\mathrm{R}\left(43^{\circ}\right.$ $\left.17^{\prime} \mathrm{N}, 2^{\circ} 23^{\prime} \mathrm{E}\right)$. Coll and subm 1972 by L Rieucau, Univ Paris IV.

\section{Gif-3384. Bordeaux, GA 28145, France $\quad 4190 \pm 110$}

Wood from alluvial fm, at Bordeaux $\left(44^{\circ} 50^{\prime} \mathrm{N}, 0^{\circ} 34^{\prime} \mathrm{W}\right)$, depth $6 \mathrm{~m}$. Coll and subm 1974 by J Alvinerie, Inst Geol Bassin d'Aquitaine, Talence, Bordeaux.

Spain

\section{Tenerife Island series, Canary Islands}

Calcareous crusts interbedded with brown soils, developed on basalt substratum, Tenerife I. Coll and subm 1975 by P Quantin, ORSTOM, Bondy. Measured to date this type of soil formation. 
Gif-3776. Los Olivos, T 5-1

$\left(28^{\circ} 06^{\prime} \mathrm{N}, 13^{\circ} 02^{\prime} \mathrm{W}\right)$, depth 70 to $85 \mathrm{~cm}$.

Gif-3777. Los Olivos, $T$ 5-2

$\left(28^{\circ} 06^{\prime} \mathrm{N}, 13^{\circ} 02^{\prime} \mathrm{W}\right)$, depth 125 to 135 , same sec as T 5-1. Comment: due to contamination problems for this type of sample, only lower limit of age is given.

Gif-3779. Bajamar, $\mathbf{T} 6$

$24,500 \pm 700$

$\left(28^{\circ} 35^{\prime} \mathrm{N}, 12^{\circ} 40^{\prime} \mathrm{W}\right)$ depth 70 to $85 \mathrm{~cm}$.

Gif-3780. Fasnia, T 7-3

$19,100 \pm 400$

$\left(28^{\circ} 13^{\prime} \mathrm{N}, 12^{\circ} 44^{\prime} \mathrm{W}\right)$, depth 395 to $410 \mathrm{~cm}$.

\section{Area Longa series, Asturias}

Peat from peat beds interstratified in solifluction flow, Area Longa beach $\left(43^{\circ} 36^{\prime} \mathrm{N}, 7^{\circ} 25^{\prime} \mathrm{W}\right)$. Coll and subm 1974-1975 by G Mary, Fac Sci, Le Mans.

Gif-3490. Area Longa, 2

$16,780 \pm 400$

From peaty level $2,30 \mathrm{~cm}$ thick, overlying solifluction flow, $3 \mathrm{~m}$ above beach level.

Gif-3489. Area Longa, 1, upper part

$15,950 \pm 500$ flow.

From upper part of peaty level 1 , ca $1 \mathrm{~m}$ thick, underlying solifluction

Gif-3488. Area Longa, 1, lower part

$\geq \mathbf{3 5 , 0 0 0}$

From lower part of level 1.

General Comment: dates point out existence of solifluction period at 16,000 BP.

\section{La Franca series, Asturias}

Organic horizons interstratified in head which forms cliff on $\mathrm{La}$ Franca beach $\left(43^{\circ} 23^{\prime} \mathrm{N}, 4^{\circ} 23^{\prime} \mathrm{W}\right)$. Samples coll and subm $1974-1975$ by $\mathrm{G}$ Mary (Mary, Medus \& Delibrias, 1977).

Gif-3051. La Franca, 3

$\mathbf{2 0 , 3 0 0} \pm \mathbf{5 0 0}$

Peaty soil. Comment: date corresponds to Early Würm III, as expected from palynology.

Gif-3501. La Franca, 7

$32,900 \pm 1600$

Paleosol, from level under Gif-3051. Comment: because of very low content of organic matter in paleosol, date must be taken as age limit rather than true age. 
Gif-3052. La Franca, 1

Peat from peaty basal level.

\section{Africa}

Morocco

\section{Imsouane, El Majni series}

Samples from sec in alluvial silty terrace, $\left(31^{\circ} 50^{\prime} \mathrm{N}, 9^{\circ} 40^{\prime} \mathrm{W}\right)$. Coll and subm 1975 by P Rognon.

Gif-3616. Imsouane, 34-3 b

$880 \pm 80$

Charcoal from kitchen midden.

Gif-3617. Imsouane, 34-3 b

$570 \pm 100$

Shells assoc with charcoal from same level as Gif-3616.

Gif-3621. Imsouane, 34-2 b

$13,900 \pm 250$

Shells (Helix sp) from sandstone level overlying typical rose-colored silty deposit.

Gif-3620. Imsouane, 34-2 a

$27,700 \pm 1100$

Shells (Helix sp) from bottom of rose-colored silty deposit.

General Comment: Gif-3621 dates important development of littoral dunes after episode of rose-colored silt deposit, at end of Soltanian Pluvial (Delibrias, Rognon \& Weisrock, 1976).

\section{Tissouirine, Ksob Wadi series}

Samples from terrace of Ksob Wadi $\left(31^{\circ} 30^{\prime} \mathrm{N}, 9^{\circ} 45^{\prime} \mathrm{E}\right)$. Coll and subm 1975-1981 by P Rognon.

Gif-3619. Ksob wadi, $S$ sand.

Charcoal from organic level in upper part of terrace under aeolian

Gif-6000. Ksob wadi, AG 66

$1380 \pm 60$

Shells (Helix sp) from shelly level at top of brown silt deposit, on sandy hill, in wadi estuary, near Essarouira.

Gif-3618. Ksob wadi, 50

$4950 \pm 120$ 6000 .

Shells (Helix sp) from shelly level, in brown silt deposit, $1 \mathrm{~m}$ under Gif-

Gif-4053. Ksob wadi, 45

$5700 \pm 120$

Shells (Helix sp) in human settlement on dune, near Ksob wadi.

General Comment: Gif-4053 dates end of arid period of sand accumulation. 
Gif-6000 and -3618 date wadi overflows during Holocene (Weisrock et al, 1985).

Gif-3615. Koudiat el Aouija

Lacustrine mussels from bottom of rose-colored silt deposit at upper part of low alluvial terrace of wadi Tensif $\left(31^{\circ} 40^{\prime} \mathrm{N}, 8^{\circ} 45^{\prime} \mathrm{W}\right)$. Coll and subm by P Rognon.

Gif-4542. Moulay Bou Selham lagoon, Kenitra

$3490 \pm 100$

Shelly level from $0.60-0.67 \mathrm{~m}$ depth in core $78001\left(34^{\circ} 58^{\prime} \mathrm{N}, 6^{\circ} 18^{\prime}\right.$ W). Coll and subm 1978 by J Moyes, Univ Bordeaux 1. Comment: dates closing of lagoon.

Other Countries

\section{Kafubu series, Shaba, Zaire}

Samples from bank of river at Kafubu $\left(12^{\circ} 53^{\prime} \mathrm{S}, 27^{\circ} 46^{\prime} \mathrm{E}\right)$. Alt $1120 \mathrm{~m}$. Coll by A Lequarré and subm 1975 by P de Maret, Mus Royal Afrique Centrale, Tervuren, Belgium.

Gif-3454. Kafubu, 16/29-6

$170 \pm 100$

Charcoal assoc with ceramics, depth $1.50-2.00 \mathrm{~m}$.

Gif-3455. Kafubu, 3/29-6

$1140 \pm 90$

Wood assoc with remains of cob-work, depth $5 \mathrm{~m}$.

Gif-3456. Kafubu, 19/29-6

$2030 \pm 100$

Wood, depth $5 \mathrm{~m}$.

General Comment: dates indicate high fluviatile sedimentation rate.

Delta of Chari R series, Chad

Organic sediments from borings in delta of Chari (ca $12^{\circ} 50^{\prime} \mathrm{N}, 14^{\circ} 30^{\prime}$ E). Coll and subm 1975 by M Mathieu, ORSTOM, N'Djamena, Chad.

Gif-3458. Boring V-18

$270 \pm 90$

Depth $4.10 \mathrm{~m}$.

Gif-3459. Boring XV-11

Modern

Depth $1.15 \mathrm{~m}$.

Gif-3460. Sec XVI-2

$510 \pm 90$

Depth $2.70 \mathrm{~m}$, in sec in river bank.

General Comment: dates are much younger than expected, which suggests very high sedimentation rate.

Gif-3424. Koren Mogodaye, Yagoua, N Cameroon

$4010 \pm 110$

Charcoal coated with iron-clay deposit in $\mathrm{B}$ horizon of pedogenic profile at top of red-sand dune $\left(10^{\circ} 17^{\prime} \mathrm{N}, 15^{\circ} 4^{\prime} \mathrm{E}\right)$. Coll and subm 1975 by $\mathrm{M}$ 
Gavaud, ORSTOM, Yaounde, Cameroon. Comment: dates fm of this type of ferrigeneous soil.

\section{Badoudi series, Garoua region, Cameroon}

Samples from Profile BEG 182 in ancient terrace of Benoué R, at Badoudi $\left(9^{\circ} 15^{\prime} \mathrm{N}, 13^{\circ} 23^{\prime} \mathrm{E}\right)$. Alt $8 \mathrm{~m}$. Profile shows succession of differentiated and leached soils, which are result from recent pedogenesis. Coll and subm 1975 by M Gavaud.

\section{Gif-3359. Badoudi, BEG 182-11 b}

$10,950 \pm 220$

Calcareous nodules, 1 to $5 \mathrm{~mm}$ diam, $4.40 \mathrm{~m}$ depth in fossil vertisol.

\section{Gif-3081. Badoudi, BEG 182-12}

$8920 \pm 160$

Gastropod shells, $4.70 \mathrm{~m}$ depth, at base level of fossil vertisol with nodules dated by Gif-3359.

General Comment: Gif-3359 and -3081 are in inverted order but date of Gif3359 obtained for nodules is probably less reliable than Gif-3081. Nevertheless dates agree with Gif-871: 10,160 $\pm 200(\mathrm{R}, 1971, \mathrm{v} 13, \mathrm{p} 241)$ age of organic matter from vertisol in same strat position, ca $20 \mathrm{~km}$ from Badoudi. This episode of pedogenesis, in W Cameroon, corresponds to humid period well dated in Africa.

\section{Badda Mountain series, Ethiopia}

Peat samples from cores in peat bog on E side of Badda Mt $\left(8^{\circ} 00^{\prime} \mathrm{N}\right.$, $39^{\circ} 45^{\prime}$ E). Alt $+4043 \mathrm{~m}$. Coll 1975 by A Street and D Lauder and subm 1976 by F Gasse.

Gif-3889. Badda Mt, S 1, Bog 3, 40-50cm $770 \pm 110$

Comment: undersized sample.

Gif-3890. Badda Mt, Bog 3, 140-150 cm

$$
2520 \pm 100
$$

Gif-3891. Badda Mt, Bog 3, 290-300cm

$$
11,500 \pm 200
$$

Gif-4684. Badda Mt, Bog 1, 40-50cm $790 \pm 80$

Gif-4685. Badda Mt, Bog 1, 90-100cm

$$
1700 \pm 90
$$

Gif-4109. Badda Mt, Bog 1, 183-200cm

Comment: undersized sample.

Gif-4108. Badda Mt, Bog 1, 232-250cm

Gif-2915. Antanetibe plain, Hauts-Plateaux, AHR 35, Madagascar

Organic horizon, $2.10 \mathrm{~m}$ depth underlying clayey soil $\left(18^{\circ} 30^{\prime} \mathrm{S}, 47^{\circ} 30^{\prime}\right.$ E). Coll and subm 1974 by C Ratsimbazafy, ORSTOM, Tananarive. 
Gif-2916. Antanetibe plain, Hauts-Plateaux, AHR 64, Madagascar

Modern

Organic horizon, $1.50 \mathrm{~m}$ depth underlying hydromorphic soil in alluvial Antanetibe plain $\left(18^{\circ} 22^{\prime} \mathrm{S}, 47^{\circ} 33^{\prime} \mathrm{E}\right)$. Coll and subm 1973 by C Ratsimbazafy. Comment: Gif-2916 and -2915 indicate very recent fossilization and rapid sedimentation rate in that region (Bourgeat \& Ratsimbazafy, 1975).

Gif-2958. Iazafo plain, ZAR 1007, Madagascar

$8110 \pm 170$

Organic horizon underlying alluvial deposit, $3 \mathrm{~m}$ thick $\left(17^{\circ} 25^{\prime} \mathrm{S}, 49^{\circ}\right.$ $16^{\prime}$ E). Coll and subm 1973 by C Ratsimbazafy.

Gif-2959. Iazafo plain, ZAR 334, Madagascar

$17,700 \pm 420$

Black organic horizon underlying clayey alluvial deposit, in low terrace, $2.80 \mathrm{~m}$ thick $\left(17^{\circ} 25^{\prime} \mathrm{S}, 49^{\circ} 17^{\prime} \mathrm{E}\right)$. Coll and subm 1973 by $\mathrm{C}$ Ratsimbazafy. Comment: Gif-2958 and 2959 indicate that weathering and concomitant low terrace fm occurred between 25,000 and 8000 BP (Bourgeat \& Ratsimbazafy, 1975).

\section{Southern Indian Ocean}

\section{Kerguelen Island series}

Peat samples subm 1974 by N Bellair, CNRS, Paris.

Gif-3543. Betsy Cove 1

$1620 \pm 90$

$\left(49^{\circ} 10^{\prime} \mathrm{S}, 70^{\circ} 12^{\prime} \mathrm{E}\right)$, depth 0.95 to $1.05 \mathrm{~m}$.

Gif-3544. Betsy Cove 2

$3340 \pm 100$

Depth 1.10 to $1.25 \mathrm{~m}$.

Gif-3545. Betsy Cove 3

$4260 \pm 110$

Depth 1.85 to $2.00 \mathrm{~m}$.

Gif-3546. Betsy Cove 4

$3840 \pm 110$

Depth 2.15 to $2.25 \mathrm{~m}$.

Gif-3542. Dante Plain

$5790 \pm 120$

$\left(49^{\circ} 38^{\prime} \mathrm{S}, 69^{\circ} 34^{\prime} \mathrm{E}\right)$, depth 2.25 to $2.35 \mathrm{~m}$.

Possession Island series, Crozet Archipelago

Peat samples subm 1972-73 by N Bellair.

Gif-2602. Branloirs Valley, 2

$840 \pm 90$

( $46^{\circ} 24^{\prime} \mathrm{S}, 51^{\circ} 45^{\prime} \mathrm{E}$ ), depth 0.95 to $1.30 \mathrm{~m}$.

$4570 \pm 130$

Gif-2460. Branloirs Valley, 1

Depth 3.50 to $3.75 \mathrm{~m}$. 
Gif-4501. American Bay

$\left(46^{\circ} 23^{\prime} \mathrm{S}, 51^{\circ} 48^{\prime} \mathrm{E}\right)$, depth 2.10 to $2.50 \mathrm{~m}$.

General Comment: for Kerguelen and Possession I series: because of relatively high sedimentation rate, studied secs do not represent long period of time and only climatic variations of Little Ice Age were indicated by pollen analysis (Roche-Bellair, 1973).

\section{South America}

\section{Los Llanos Centrales series, Venezuela}

In central plains of Venezuela, $+100 \mathrm{~m}$ above msl, dune fms extend over large area from $6^{\circ}$ to $9^{\circ} 15^{\prime} \mathrm{N}$ and $64^{\circ}$ to $70^{\circ} \mathrm{W}$. These fossil dunes, 10 to $15 \mathrm{~m}$ high, presently fixed by grass, contain some paleosol levels. Samples from these organic horizons were coll by boring and subm 1974 by $\mathrm{P} R$ Morales, Fac Ciencas, Univ Central Venezuela, Caracas.

Gif-3331. Llanos Centrales, M-27 A

$2290 \pm 90$ W).

Black sandy level with vegetal debris, depth $2.05 \mathrm{~m}\left(7^{\circ} 33^{\prime} \mathrm{N}, 69^{\circ} 15^{\prime}\right.$

Gif-3332. Llanos Centrales, M-33 B

$3700 \pm 110$ W).

Black sandy level with vegetal debris, depth $1.60 \mathrm{~m}\left(7^{\circ} 34^{\prime} \mathrm{N}, 69^{\circ} 16^{\prime}\right.$

Gif-3333. Llanos Centrales, M-35 C

$4130 \pm 240$ W).

Brown sandy level with vegetal debris, depth $1.20 \mathrm{~m}\left(7^{\circ} 29^{\prime} \mathrm{N}, 69^{\circ} 15^{\prime}\right.$

Gif-3335. Llanos Centrales, M-32 B

$5860 \pm 270$ W).

Silty level with vegetal and charcoal debris, depth $2 \mathrm{~m}\left(8^{\circ} 32^{\prime} \mathrm{N}, 67^{\circ} 32^{\prime}\right.$

Gif-3336. Llanos Centrales, M-19-1-aP

$11,000 \pm 450$

Silty level with vegetal remains, depth $4.20 \mathrm{~m}\left(8^{\circ} 32^{\prime} \mathrm{N}, 67^{\circ} 33^{\prime} \mathrm{W}\right)$.

General Comment (PRM): dates succession of arid periods with sand accumulation and moist periods with soil development since 11,000 BP. Dunes imply existence of vast desert in that region during last glaciation.

\section{North America}

\section{Owl River series, Baffin Island, Canada}

Peat from $3 \mathrm{~m}$ river-cut exposure of Owl R, Pangnirtung Pass $\left(66^{\circ} 45^{\prime}\right.$ $\left.\mathrm{N}, 64^{\circ} 42^{\prime} \mathrm{W}\right)$. Coll by $\mathrm{G} \mathrm{H}$ Miller and subm 1974 by J T Andrews, Univ Colorado, Boulder.

Gif-3493. Owl River, $155-160 \mathrm{~cm}$

$1870 \pm 90$ 


\section{Gif-3494. Owl River, 289-294cm}

Comment (JTA): peat underlying $1.5 \mathrm{~m}$ of niveo-aeolian sand that reflects increased aridity in $\mathrm{N} \mathrm{sec}$ Pangnirtung Pass since 2660 BP.

\section{Gif-4044. Prudhoe Bay, N Alaska}

$$
6480 \pm 130
$$

Peat from underlying blue clay exposed in coastal bluff, Prudhoe Bay $\left(70^{\circ} 18^{\prime} \mathrm{N}, 148^{\circ} 23^{\prime} \mathrm{W}\right)$, alt ca $0.2 \mathrm{~m}$ above msl. Peat is covered by $20 \mathrm{~cm}$ dead vegetal matter, $20 \mathrm{~cm}$ alternating dark and light colored silts and $40 \mathrm{~cm}$ dark brownish-black organic-rich clay. Coll and subm 1974 by J T Andrews. Comment (JTA): strat suggests marine transgression or clay solifluction when sea was lower than at present. Pollen analysis shows Cyperaceae dominates, but Betula, Alnus and Picea are present in significant amounts.

\section{Kuparuk River series, Alaska}

Peat from river-bank exposure of Kuparuk R $\left(70^{\circ} 19^{\prime} \mathrm{N}, 148^{\circ} 57^{\prime} \mathrm{W}\right)$, ca $6 \mathrm{~m}$ above msl. Coll and subm 1974 by J T Andrews.

$$
\begin{array}{lll}
\text { Gif-3667. } & \text { Kuparuk R, } 74 \mathrm{~cm} & 2300 \pm 110 \\
\text { Gif-3666. } & \text { Kuparuk R, } 195-205 \mathrm{~cm} & 3470 \pm 100
\end{array}
$$

General Comment (JTA): river gravels were overlain by overbank silts and clays and succeeded by peats and sands after $3500 \mathrm{BP}$. Shortly after $2300 \mathrm{BP}$, site was dominated by eolian sediments. Pollen analysis shows Cyperaceae, Gramineae, and Alnus, Betula, Pinus, Picea.

\section{Gif-3668. Putuligayuk River, Alaska}

$3270 \pm 100$

Peaty clay sample from base of river-bank exposure along Putuligayuk $\mathrm{R}\left(70^{\circ} 14^{\prime} \mathrm{N}, 148^{\circ} 44^{\prime} \mathrm{W}\right)$, ca $4 \mathrm{~m}$ above msl. Coll and subm 1974 by $\mathrm{J} \mathrm{T}$ Andrews.

\section{Sagavanirktok River series, Alaska}

Peat samples from slumped block of $\mathbf{E}$ facing slope of Sagavanirktok $\mathbf{R}$ $\left(70^{\circ} 13^{\prime} \mathrm{N}, 148^{\circ} 22^{\prime} \mathrm{W}\right)$. Coll and subm 1974 by J T Andrews.

Gif-3665. Sagavanirktok R

Near surface.

Gif-3664. Sagavanirktok R

Depth $1.35 \mathrm{~m}$.

\section{Marine Samples}

\section{Large manganese nodules series}

Carbonate inclusions in fluorapatite core of large manganese nodules from Pacific Ocean $\left(16^{\circ} 28^{\prime} \mathrm{S}, 146^{\circ} 34^{\prime} \mathrm{W}\right), 1000 \mathrm{~m}$ depth. Samples subm 1973 by C Lalou, CFR-CNRS, Gif sur Yvette, in connection with Mn nod- 
ules growth rate and Mn chemistry in sea studies (Lalou et al, 1973; Lalou, Brichet \& Bonté, 1976).

\section{Gif-2314. Nodule D IV A}

Weight $16.5 \mathrm{~kg}$.

\section{Gif-2751. Nodule DO 23 F}

$15,700 \pm 400$ $\delta^{13} \mathrm{C}=+1.2 \%$

$\mathbf{2 5 , 8 0 0} \pm \mathbf{8 0 0}$ $\delta^{13} C=+2.3 \%$

\section{Weight $9.5 \mathrm{~kg}$.}

General Comment: these values cannot be considered true ages. Presence of ${ }^{14} \mathrm{C}$ indicates either continual exchange of $\mathrm{C}$ with sea water or exchange during abrupt recrystallization of core. Undersized samples.

\section{Mn deposit series}

Coral samples dredged from Mid-Atlantic ridge $\left(36^{\circ} 46^{\prime} \mathrm{N}, 35^{\circ} 15^{\prime} \mathrm{W}\right)$, between 2850 and $2500 \mathrm{~m}$, near hydrothermal field, by R Hekinian, COBCNEXO. Subm 1975 by C Lalou.

\section{Gif-3440. $\quad$ CH $31 n^{\circ} 4$}

$3730 \pm 230$

Inside part of coral branch (Isis sp) which shows Mn annular ring, $6 \mu \mathrm{m}$ thick, between two growth phases. Comment: x-ray analysis shows $100 \%$ calcite.

\section{Gif-3441. $\quad$ CH 31 n $^{\circ} 2$}

$3550 \pm 230$

Coral from same branch as Gif-3440, outside Mn ring. Comment: x-ray analysis shows $100 \%$ calcite. Dates of Gif-3440 and -3441 , within statistical margin, show Mn was deposited very quickly.

\section{Gif-3958. CH 31, DR 5 B}

$6760 \pm 160$

Coral branch (Desmophyllum sp) with external Mn deposit $6 \mu \mathrm{m}$ thick. Comment: x-ray analysis shows $10 \%$ calcite.

General Comment: undersized samples.

Gif-3636. Coral branch, DO 11 (1), Pacific Ocean

Internal part of coral branch, diam $5 \mathrm{~mm}$, underlying manganese deposit, from Pacific Ocean, near Tuomatu I. Coll by CNEXO and subm 1975 by C Lalou ( $17^{\circ} 13^{\prime} \mathrm{S}, 145^{\circ} 44^{\prime}$ W) $1140 \mathrm{~m}$ depth. Comment: undersized sample.

\section{Gif-3731. Mid-Atlantic Ridge $\quad \geq \mathbf{2 8 , 0 0 0}$}

Travertine inside Mn-Fe-carbonate geode, coll 1974 during FAMOUS expedition by CYANA submersible on surface sediment of Mid-Atlantic Ridge, depth $3000 \mathrm{~m}, 8 \mathrm{~m}$ from hydrothermal vents $\left(36^{\circ} 56^{\prime} \mathrm{N}, 33^{\circ} 04^{\prime} \mathrm{W}\right)$. Subm 1975 by C Lalou.

\section{La Romanche Trench series, Atlantic Ocean}

Organic fraction of sediment from marine core $71-08,770 \mathrm{~cm}$ long $\left(0^{\circ}\right.$ $14^{\prime} \mathrm{S}, 18^{\circ} 37^{\prime} \mathrm{W}$ ), depth $7500 \mathrm{~m}$. Subm 1974 by C Lalou. 
Undersized sample.

Gif-3426. Core 71-08, 71-75cm

Gif-3442. Core 71-08, $403-407 \mathrm{~cm}$

Gif-4247. Core 71-08, 664-674cm

General Comment: dates help to calculate very high sedimentation rate, ca $50 \mathrm{~cm} / 1000 \mathrm{yr}$, which is due to funnel shape of trench (Bonté $e t a l, 1982)$. Undersized samples.

\section{Atlantic abyssal plain series}

Marine sediments were coll by box cores, in 1974 during MIDLANTE expedition in Atlantic abyssal plain. Samples subm 1975 by A Monaco, Centre Univ, Perpignan. Carbonate fraction $\geq 40 \mu$ was dated.

Gif-3754. Atlantic abyssal plain, KR 02, 1 $2740 \pm 200$ $\left(18^{\circ} 24^{\prime} \mathrm{N}, 24^{\circ} 34^{\prime} \mathrm{W}\right)$, depth $3794 \mathrm{~m}$, surface sediment, 0 to $1 \mathrm{~cm}$.

Gif-3790. Atlantic abyssal plain, KR 02, 3

$3040 \pm 200$

2 to $3 \mathrm{~cm}$ depth.

Gif-3791. Atlantic abyssal plain, KR 03, 1 $2660 \pm 200$

$\left(19^{\circ} 36^{\prime} \mathrm{N}, 27^{\circ} 26^{\prime} \mathrm{W}\right)$, depth $4791 \mathrm{~m}$, surface sediment, 0 to $1 \mathrm{~cm}$.

General Comment: high sedimentation rate deduced from Gif-3754 and -3790 is probably due to turbidite currents, indicated by sedimentologic and mineralogic studies.

\section{Blanc du Four series, W Atlantic}

Carbonate fraction of sediment cores in organogenic calcareous sand bank (hydraulic sand dunes). Samples subm 1975 by J P Le Gorgeu, CNEXO, Brest.

Gif-3679. Blanc du Four, 35 F 1

From 1 st $\mathrm{m}$ of core $35 \mathrm{~F}\left(48^{\circ} 38^{\prime} \mathrm{N}, 4^{\circ} 54^{\prime} \mathrm{W}\right)$, depth $43 \mathrm{~m}$.

Gif-3497. Blanc du Four, 35 F 10

From 10 th $\mathrm{m}$ of core $35 \mathrm{~F}$.

Gif-3680. Blanc du Four, 41 F 1

From $1 \mathrm{st} \mathrm{m}$ of core $41 \mathrm{~F}\left(48^{\circ} 31^{\prime} \mathrm{N}, 4^{\circ} 53^{\prime} \mathrm{W}\right)$, depth $60 \mathrm{~m}$.

Gif-3498. Blanc du Four, 41 F 9

From 9 th $\mathrm{m}$ of core $41 \mathrm{~F}$.

General Comment: dated to estimate sand accumulation rate in order to exploit dunes, but ages are not significant; sediments were probably reworked by fm process of hydraulic dunes. 


\section{Miscellaneous Samples}

\section{Soil series}

Study of evolution rate of organic matter in two types of Mediterranean soils, on calcareous substratum, was made at Massif de la Gardiole $\left(43^{\circ} 29^{\prime} \mathrm{N}, 3^{\circ} 44^{\prime} \mathrm{E}\right.$ ), alt $210 \mathrm{~m}$ (calcic-ferrasiallitic soil A), and at Bois Doscare $\left(43^{\circ} 37^{\prime} \mathrm{N}, 3^{\circ} 57^{\prime} \mathrm{E}\right.$ ), alt $55 \mathrm{~m}$ (non-calcic-ferrasiallitic soil B), Hérault (Bottner, 1972). Samples (Table 7) were coll 1973 and chemical preparation made by P Bottner, Centre d'Etudes Phytosociolog écolog, Montpellier, France. Different separated fractions result from successive hydrolysis, (F II and F IV), and from alkaline extractions (F III).

General Comment: different components of $\mathrm{O} \mathrm{M}$ in surface horizons of both soils are young which means that turnover of $\mathrm{OM}$ is rapid in these soils. Presence of bomb ${ }^{14} \mathrm{C}$ makes it possible to establish residence time of $\mathrm{C}$ in $\mathrm{A}$ horizons at most $15 \mathrm{yr}$ and is shorter in B Cryptomull than in A Calcic mull, as expected.

TABLE 7

Massif de la Gardiole and Bois Doscare

\begin{tabular}{|c|c|c|c|c|}
\hline \multicolumn{5}{|c|}{ Soil A: Calcic mull } \\
\hline Sample & Fraction & $\begin{array}{c}{ }^{14} \mathrm{C} \\
\text { activity } \\
(\triangle \% 0)\end{array}$ & $\begin{array}{c}\text { Age } \\
\text { (yr BP) }\end{array}$ & Horizon \\
\hline $\begin{array}{r}\text { Gif-3234 } \\
-3235 \\
-3236 \\
-3237 \\
\end{array}$ & $\begin{array}{c}\text { Total OM } \\
\text { F II } \\
\text { F III } \\
\text { F IV }\end{array}$ & $\begin{array}{l}+142 \pm 10 \\
+137 \pm 10 \\
+191 \pm 10 \\
+139 \pm 10\end{array}$ & & $\begin{array}{l}\text { Al } \\
2-10 \mathrm{~cm}\end{array}$ \\
\hline-3552 & Total OM & & $210 \pm 80$ & $\begin{array}{l}\text { A3 } \\
10-35 \mathrm{~cm}\end{array}$ \\
\hline-3553 & Total OM & & $1940 \pm 100$ & $\begin{array}{l}\mathrm{B} \\
70-120 \mathrm{~cm}\end{array}$ \\
\hline \multicolumn{5}{|c|}{ Soil B: Cryptomull } \\
\hline $\begin{array}{r}\text { Gif-3238 } \\
-3239 \\
-3240 \\
-3241\end{array}$ & $\begin{array}{c}\text { Total OM } \\
\text { F II } \\
\text { F III } \\
\text { F IV }\end{array}$ & $\begin{array}{l}+366 \pm 10 \\
+224 \pm 10 \\
+256 \pm 10 \\
+153 \pm 10\end{array}$ & & $\begin{array}{l}\text { A1 } \\
3-12 \mathrm{~cm}\end{array}$ \\
\hline
\end{tabular}

\section{Fossil hydrocarbon in marine organism series}

Measurements of ${ }^{14} \mathrm{C}$ activity of shells and algae living in estuary of Rance $\mathrm{R}\left(48^{\circ} 60^{\prime} \mathrm{N}, 2^{\circ} \mathrm{W}\right)$, in estuary of Loire $\mathrm{R}\left(47^{\circ} 17^{\prime} \mathrm{N}, 2^{\circ} 12^{\prime} \mathrm{W}\right)$ and in Aber Benoit $\left(48^{\circ} 33^{\prime} \mathrm{N}, 3^{\circ} 27^{\prime} \mathrm{W}\right.$ ) were studied to distinguish between fossil organic carbon due to petroleum and modern organic carbon, in living marine organisms (Table 8). Samples were coll and subm 1975 and 1978 by P Renault and J C Roussel, Inst Français Pétrole, Rueil-Malmaison. Extraction and identification of different dated fractions were done by IFP.

General Comment: results indicate importance of carbon of fossil origin in marine organisms. Gif-4832 shows that totality of hydrocarbon is fossil in 
TABLE 8

Fossil hydrocarbon in marine organisms

\begin{tabular}{|c|c|c|c|c|c|}
\hline Sample & Material & $\begin{array}{l}\delta^{14} \mathrm{C} \\
(\% 0)\end{array}$ & $\begin{array}{l}\delta^{13} \mathrm{C} \\
(\% 0)\end{array}$ & $\begin{array}{l}\Delta^{14} \mathrm{C} \\
(\% 0)\end{array}$ & $\begin{array}{c}\text { Fossil } \\
\text { carbon } \\
(\%) \\
\end{array}$ \\
\hline $\begin{array}{r}\text { Gif-3771 } \\
-3749\end{array}$ & $\begin{array}{l}\text { Rance estuary, Algua, ref } 1975 \\
\text { Rance estuary, Zostera, cellulose } \\
\text { ref } 1975\end{array}$ & $\begin{array}{l}+194 \\
+186\end{array}$ & $\begin{array}{l}-16.1 \\
(-16)\end{array}$ & $\begin{array}{r}+172.8 \pm 10 \\
+164 \pm 10\end{array}$ & \\
\hline-3887 & $\begin{array}{l}\text { Rance estuary, Laminaria, } \\
\text { cellulose ref } 1975\end{array}$ & +195 & $(-16)$ & $+173 \pm 10$ & \\
\hline-3751 & $\begin{array}{l}\text { Rance estuary, Laminaria, lipid } \\
\text { fraction }\end{array}$ & +196 & $(-16)$ & $+174 \pm 22$ & 0 \\
\hline-4009 & $\begin{array}{l}\text { Rance estuary, Laminaria, } \\
\text { hydrocarbon in hexane, } 1\end{array}$ & -282 & $(-16)$ & $-295 \pm 25$ & 40 \\
\hline-4041 & $\begin{array}{l}\text { Rance estuary, Laminaria, } \\
\text { hydrocarbon in benzene }\end{array}$ & -233 & $(-16)$ & $-233 \pm 30$ & 36 \\
\hline-4537 & $\begin{array}{l}\text { Rance estuary, Laminaria, } \\
\text { hydrocarbon in hexane, } 2\end{array}$ & -58.5 & $(-16)$ & $-75 \pm 10$ & 21 \\
\hline-4432 & $\begin{array}{l}\text { Loire estuary, mussel shells, ref } \\
1978\end{array}$ & +151 & $(\mathrm{ca} \mathrm{0})$ & $+93 \pm 7$ & \\
\hline-4591 & $\begin{array}{l}\text { Loire estuary, mussel flesh, } \\
\text { hydrocarbon }\end{array}$ & +46 & -27.6 & +51.5 & 4 \\
\hline-4590 & $\begin{array}{l}\text { Loire estuary, } 0 \mathrm{M} \text { in marine } \\
\text { sediments, hydrocarbon }\end{array}$ & -612 & -23.9 & $-611 \pm 7$ & \\
\hline-4829 & $\begin{array}{l}\text { Aber Benoit, oyster shells, ref } \\
1978\end{array}$ & +161 & $(0)$ & $+103 \pm 5$ & \\
\hline-4830 & $\begin{array}{l}\text { Aber Benoit, oyster flesh, } \\
\text { hydrocarbon in hexane }\end{array}$ & -212 & -218 & $-217 \pm 25$ & 29 \\
\hline-4831 & $\begin{array}{l}\text { Aber Benoit, oyster flesh, } \\
\text { hydrocarbon in methanol }\end{array}$ & +74 & -21.3 & $+66 \pm 24$ & 4 \\
\hline-4832 & $\begin{array}{l}\text { Aber Benoit, } 0 \mathrm{M} \text { in marine sed } \\
\text { hydrocarbon in hexane }\end{array}$ & & -32.7 & $>-980$ & $>98$ \\
\hline
\end{tabular}

organic matter of sediments of Aber Benoit. Moreover, ${ }^{14} \mathrm{C}$ measurements help calculate fossil carbon percentage in different separated fractions and improve chemical extraction of hydrocarbon.

Cuenca del Lago de Valencia series, San Joaquim-El Ereigue, Venezuela

Organic sediments from Lago de Valencia $\left(\right.$ ca $10^{\circ} \mathrm{N}, 68^{\circ} \mathrm{W}$ ), alt $+1000 \mathrm{~m}$. Subm 1977 by G R Sieffermann.

\section{Gif-4353. Lago de Valencia, depth 4.5m}

$980 \pm 100$

Gif-4354. Lago de Valencia, depth $7.5 \mathrm{~m}$

$5900 \pm 100$

General Comment: dates help evalute erosion rate in basin.

Gif-4355. Rio Guarapiche, Chiguata, Venezuela

$2700 \pm 150$

Organic level in recent terrace of $\mathrm{R}$ Guarapiche (ca $\left.9^{\circ} \mathrm{N}, 64^{\circ} \mathrm{W}\right), 115$ to $117 \mathrm{~cm}$ under surface. Subm 1977 by G R Sieffermann, ORSTOM, Paris.

Gif-2890. Bansbari, Kathmandu, Nepal

Lignite, $3 \mathrm{~m}$ thick, in sand pit underlying gray and yellow silty sand, $6 \mathrm{~m}$ thick. Coll and subm 1973 by J Hubschman, Inst Geog, Univ Toulouse. Comment: date is min for fill of this lacustrine depression. 


\section{REFERENCES}

Angelier, J, Cadet, J P, Delibrias, G, Fourniguet, J, Gigout, M, Guillemin, M, Hogrel, M T, Lalou, C and Pierre, G, 1976, Les déformations du Quaternaire marin, indicateurs néotectoniques. Quelques exemples méditerranéens: Rev Géog Phys Géol Dynamique, v 18, p $427-448$.

Bartholi, J M, 1975, Recherches historiques et archéologiques sur les chapelles basse et haute de l'Eglise Saint-Martin de Palaiseau. La découverte de la tombe des Arnauld: Bull Soc Hist Archaeol Corbeil, Etampes and Rurepoix.

Bégouën, R, Clottes, J, Giraud, J P and Rouzaud, F, 1984, Compléments à la grande plaquette gravée d'Entene: Bull Soc Préhist Fr, v 81, no. 3, p 142-148.

Bonté, Ph, Labeyrie, L D, Dudley, W C, Blanc, P L, Berthois, L, Hékinian, R and Duplessy, J C, 1982, Morphology and tectonics of the Romanche transform fault: high resolution mapping and precision sampling of the northern slope: Oceanol Acta, v 5, no. 2, p 235240 .

Bottner, P, (ms) 1972, Evolution des sols en milieu carbonaté: Sci thesis, Univ Montpellier, $156 \mathrm{p}$.

Bourgeat, F and Ratsimbazafy, C, 1975, Retouches à la chronologie du Quaternaire continental de Madagascar: Conséquences sur la pédogenèse: Bull Soc Géol Fr, v 7, no. 4, p 554561 .

Chauchat, C, 1976, The Paijan complex, Pampa of Cupisnique, Peru, Nawpa Pacha 13, 85104, Tawantinsuyu K'Uzkiy Paqar ichisqa: Inst Andean Studies, Berkeley, California. (ms) 1982, Le Paijanien du désert de Cupisnique. Recherches sur l'occupation préhistorique de la côte nord du Pérou au début de l'Holocène: Thesis Doc ès Sci, Univ Bordeaux X, $719 \mathrm{p}$.

Clottes, J, 1985, Informations archéologiques: Gallia-Préhist, v 28, no. 2, p 331-371.

Clottes, J and Giraud, J P, 1985, Le gisement magdalenien ancien et solutréen du Cuzoul à Vers (Lot): Bull Soc Préhist Fr, v 82, no. 1, p 5-6.

Delattre, A and Nicolardot, J P, 1976, Nouvelles datations par le carbone 14 et analyse chimique des matériaux de l'habitat fortifié néolithique de Myard à Vitteaux (Côte d'Or): Bull Soc Préhist Fr, no. 2, p 51-57.

Delibrias, G, Gasse, F and Rognon, P, 1973, Existence de lacs importants au Pleistocène supérieur (34.000-23.000 BP) dans l'Afar méridional (Ethiopie): Comptes rendus Acad Sci, v 277, p 2633-2636.

Delibrias, G, Guillier, M T and Labeyrie, J, 1970, Gif natural radiocarbon measurements V: Radiocarbon, v 12, no. 2, p 421-443. 213-254. 1971 , Gif natural radiocarbon measurements VI: Radiocarbon, v 13, no. 2, p 280-320. 1972, Gif natural radiocarbon measurements VII: Radiocarbon, v 14, no. 2, p 15-94. 291-343. 1982, Gif natural radiocarbon measurements IX: Radiocarbon, v 24, no. 3, p 68.

Delibrias, G, Rognon, P and Weisrock, A, 1976, Datation de plusieurs épisodes à "limons roses" dans le Quaternaire récent de l'Atlas Atlantique Marocain: Comptes rendus Acad
Sci, v 282, p 593-596.

De Maret, P, 1982, New survey of archaeological research and dates for $\mathrm{W}$ Central and $\mathrm{N}$ Central Africa: Jour Afr Hist, v 23, p 1-15.

Evin, J, Maréchal, J and Marien, G, 1985, Lyon natural radiocarbon measurements X: Radiocarbon, v 27, no. 2B, p 386-354.

Gasse, F, (ms) 1975, L'évolution des lacs de l'Afar centrale (Ethiopie et TFAI) du Plio-Pléistocène à l'Actuel. Reconstitution des paléomilieux lacustres à partir de l'étude des diatomées: Thesis, Univ Paris, 552 p.

$$
1978 .
$$

Gasse, F and Delibrias, G, 1976, Les lacs de l'Afar central (Ethiopic et TFAI) au Pleistocène supérieur: Paleoclimatology of Lake Biwa and Japanese Pleistocene, v 4, no. 155, p 529575.

Gillespie, R, Street-Perrott, F A and Switsur, R, 1983, Post-glacial arid episodes in Ethiopia have implications for climatic prediction: Nature, v 306, p 680-683.

Gratien, B, 1974, Les Nécropoles Kerma de Saï, II: Cahiers Recherches Inst Papyrologie Egyptologie Lille, no. 2, p 53-74.

Guilaine, J, 1979, L'abri Jean Cros: Toulouse, Centre d'Anthropol Soc Rurales Ed, 461 p.

Hugot, G, 1977, Tichitt (Aouker): Mém Inst Mauritanien Recherche Sci, v 1, 190 p. 
Hurtrelle, J and Piningre, J F, 1978, Datation radiocarbone du Cerny des Sablins à Etaples (Pas de Calais): Bull Soc Préhist Fr, v 75, no. 3, p 83-86.

Klein, J, Lerman, J C, Damon, P E and Ralph, E K, 1982, Calibration of radiocarbon dates: Tables based on the consensus data of the Workshop on Calibrating the Radiocarbon Time Scale: Radiocarbon, v 24, no. 2, p 103-150.

Lalou, C, Delibrias, G, Brichet, E and Labeyrie, J, 1973, Existence de carbone 14 au centre de deux nodules de manganèse du Pacifique. Ages carbone 14 et thorium 230 de ces nodules: Comptes rendus Acad Sci Paris, v 276, p 3013-3015.

Lalou, C, Brichet, E and Bonté, P, 1976, Some new data in the genesis of manganese nodules, in Internatl cong geol, Sydney, Geol geochem of manganese, v 3: Budapest, House of Hungarian Acad Sci Pub.

Martin, L, Suguio, K, Flexor, J M, Bittencourt, A and Vilas-Boas, G, 1979-1980, Le Quaternaire marin brésilien (Littoral Pauliste, Sud Fluminense et Bahianais: Cahiers ORSTOM, Geol ser, v 11, no. 1, p 95-124.

Mary, G, Medus, J and Delibrias, G, 1977, Documents sur l'évolution de la flore du littoral Nord Espagnol au Würm, in Recherches fr Quaternaire, INQUA: Bull AFEQ, supp v 1, no. $50, \mathrm{p} 23-31$.

Pautreau, J P, 1975, Datations radiocarbone de l'Artenac du Camp Allaric à Aslonnes (Vienne): Bull Soc Préhist Fr, CRSM, v 72, no. 1, p 24.

Petit-Maire, N and Casta, L, 1977, Un paleolac du N O mexicain, La Playa San Bartolo, in Recherches fr Quaternaire, INQUA 1977: Bull AFEQ, v 1, no. 50, p 303-322.

Piningre, J F and Bréart, B, 1976, La sépulture collective de Vers-sur-Selles (Somme): Cahiers Archéol Picardie, no. 3, p 29-37.

Plumet, P, 1977, Le peuplement prehistorique du Nouveau-Quebec: Geog phys Quaternaire (Montreal), v XXXI, no. 1-2, p 185-189.

1985, Archéologie de l'Ungava: Le site de la pointe aux Belougas et les maisons longues dorsetiennes: Colln Paleo Quebec, no. 18, p 13-471.

Pomel, R, (ms) 1979, Géographie physique de la Basse Côte d'Ivoire: Thesis, Univ Caen, France, $623 \mathrm{p}$

Robin, C, (ms) 1981, Relations volcanologie-magmatologie-géodynamique: Application au passage entre volcanismes alcalin et andesitique dans le Sud Mexicain: Thesis, Univ Clermont-Ferrand II, France, 533 p.

Roche-Bellair, N, 1973, Palynologie et datation d'une coupe de l'Ile de la Possession (Archipel des Crozet): Comptes rendus Acad Sci Paris, v 276-D, p 713-716.

Rodriguez, G, 1976, La grotte de Camprafaud, Ferrières Poussarou, Hérault, Dernières datations ${ }^{14} \mathrm{C}$ : Bull Soc Préhist Fr, CRSM, no. 6, p 190-191.

1983, La grotte de Camprafaud: Montpellier, Arceaux Ed, 415 p.

Sacchi, D, 1973, Gisements de l'Aude: Etudes préhist, v 7, p 21-24.

Talbot, M R, 1980, Holocene changes in tropical wind intensity and rainfall: Evidence from southeast Ghana: Quaternary Research, v 16, p 201-220.

Talbot, M R and Delibrias, G, 1977, Holocene variations in the level of Lake Bosumtwi, Ghana: Nature, v 268, p 722-724.

1980, A new Late Pleistocene-Holocene water-level curve for lake Bosumtwi, Ghana: Earth Planetary Sci Letters, v 47, p 336-344.

Talbot, M R, Livingstone, D A, Palmer, P G, Maley, J, Melack, J M, Delibrias, G and Gulliksen, S, 1984, Preliminary results from sediment cores from lake Bosumtwi, Ghana, in Coetzee, $\mathrm{J}$ A, Van Zinderen, E M and Bakker, S R, eds, Palaeocology of Africa and the surrounding island: Rotterdam, A A Balkema, p 173-192.

Tessier, M, 1983, La céramique de la fin de l'Age du Fer en Pays de Retz: Bull Soc Préhist Fr, v 80 , no. 7, p 221-224

Vercoutter, J, 1974, Saï 1972-1973: Cahiers Recherches Inst Papyrologie Egyptologie Lille, no. 2, p 13-26.

Weisrock, A, Delibrias, G, Rognon, P and Coude-Gaussen, G, 1985, Variations climatiques et morphogenèse du Maroc Atlantique $\left(30^{\circ}-33^{\circ} \mathrm{N}\right)$ à la limite Pléistocène-Holocène: Bull Soc Géol Fr, v 8, no. 1, p 565-569. 
